UCD-2002-10

SCIPP-02/10

hep-ph/0207010

July 2002

\title{
The CP-conserving two-Higgs-doublet model: the approach to the decoupling limit
}

\author{
John F. Gunion ${ }^{1}$ and Howard E. Haber ${ }^{2}$ \\ 1 Davis Institute for High Energy Physics \\ University of California, Davis, CA 95616, U.S.A. \\ ${ }^{2}$ Santa Cruz Institute for Particle Physics \\ University of California, Santa Cruz, CA 95064, U.S.A.
}

\begin{abstract}
A CP-even neutral Higgs boson with Standard-Model-like couplings may be the lightest scalar of a two-Higgs-doublet model. We study the decoupling limit of the most general CP-conserving two-Higgs-doublet model, where the mass of the lightest Higgs scalar is significantly smaller than the masses of the other Higgs bosons of the model. In this case, the properties of the lightest Higgs boson are nearly indistinguishable from those of the Standard Model Higgs boson. The first nontrivial corrections to Higgs couplings in the approach to the decoupling limit are also evaluated. The importance of detecting such deviations in precision Higgs measurements at future colliders is emphasized. We also clarify the case in which a neutral Higgs boson can possess Standard-Modellike couplings in a regime where the decoupling limit does not apply. The two-Higgs-doublet sector of the minimal supersymmetric model illustrates many of the above features.
\end{abstract}




\section{INTRODUCTION}

The minimal version of the Standard Model (SM) contains one complex Higgs doublet, resulting in one physical neutral CP-even Higgs boson, $h_{\mathrm{SM}}$, after electroweak symmetry breaking (EWSB). However, the Standard Model is not likely to be the ultimate theoretical structure responsible for electroweak symmetry breaking. Moreover, the Standard Model must be viewed as an effective field theory that is embedded in a more fundamental structure, characterized by an energy scale, $\Lambda$, which is larger than the scale of EWSB, $v=246 \mathrm{GeV}$. Although $\Lambda$ may be as large as the Planck scale, there are strong theoretical arguments that suggest that $\Lambda$ is significantly lower, perhaps of order $1 \mathrm{TeV}$ [1]. For example, $\Lambda$ could be the scale of supersymmetry breaking [2, 3, 4], the compositeness scale of new strong dynamics [5], or associated with the inverse size of extra dimensions [6]. In many of these approaches, there exists an effective low-energy theory with elementary scalars that comprise a non-minimal Higgs sector 7]. For example, the minimal supersymmetric extension of the Standard Model (MSSM) contains a scalar Higgs sector corresponding to that of a twoHiggs-doublet model (2HDM) 8, 9]. Models with Higgs doublets (and singlets) possess the important phenomenological property that $\rho=m_{W} /\left(m_{Z} \cos \theta_{W}\right)=1$ up to finite radiative corrections.

In this paper we focus on a general 2HDM. There are two possible cases. In the first case, there is never an energy range in which the effective low-energy theory contains only one light Higgs boson. In the second case, one CP-even neutral Higgs boson, $h$, is significantly lighter than a new scale, $\Lambda_{2 H D M}$, which characterizes the masses of all the remaining $2 \mathrm{HDM}$ Higgs states. In this latter case, the scalar sector of the effective field theory below $\Lambda_{2 \mathrm{HDM}}$ is that of the SM Higgs sector. In particular, if $\Lambda_{2 \mathrm{HDM}} \gg v$, and all dimensionless Higgs self-coupling parameters $\lambda_{i} \lesssim \mathcal{O}(1)$ [see eq. (10)], then the couplings of $h$ to gauge bosons and fermions and the $h$ self-couplings approach the corresponding couplings of the $h_{\mathrm{SM}}$, with the deviations vanishing as some power of $v^{2} / \Lambda_{2 \mathrm{HDM}}^{2}[10]$. This limit is called the decoupling limit [1]], and is one of the main subjects of this paper.

The purpose of this paper is to fully define and explore the decoupling limit of the 2HDM. ${ }^{1}$ We will explain the (often confusing) relations between different parameter sets (e.g., Higgs

\footnotetext{
${ }^{1}$ Some of the topics of this paper have also been addressed recently in ref. [12].
} 
masses and mixing angles vs. Lagrangian tree-level couplings) and give a complete translation table in Appendix A. We then make one simplifying assumption, namely that the Higgs sector is CP-conserving. (The conditions that guarantee that there is no explicit or spontaneous breaking of $\mathrm{CP}$ in the 2HDM are given in Appendix $\mathrm{B}$. The more general CP-violating 2HDM is treated elsewhere [13, 14].) In the CP-conserving 2HDM, there is still some freedom in the choice of Higgs-fermion couplings. A number of different choices have been studied in the literature [7, 15]: type-I, in which only one Higgs doublet couples to the fermions; and type-II, in which the neutral member of one Higgs doublet couples only to up-type quarks and the neutral member of the other Higgs doublet couples only to down-type quarks and leptons. For Higgs-fermion couplings of type-I or type-II, treelevel flavor-changing neutral currents (FCNC) mediated by Higgs bosons are automatically absent [16]. Type-I and type-II models can be implemented with an appropriately chosen discrete symmetry (which may be softly broken without dire phenomenologically consequences). The type-II model Higgs sector also arises in the MSSM. In this paper, we allow for the most general Higgs-fermion Yukawa couplings (the so-called type-III model [17]). For type-III Higgs-fermion Yukawa couplings, tree-level Higgs-mediated FCNCs are present, and one must be careful to choose Higgs parameters which ensure that these FCNC effects are numerically small. We will demonstrate in this paper that in the approach to the decoupling limit, FCNC effects generated by tree-level Higgs exchanges are suppressed by a factor of $\mathcal{O}\left(v^{2} / \Lambda_{2 \mathrm{HDM}}^{2}\right)$.

In Section 2, we define the most general CP-conserving 2HDM and provide a number of useful relations among the parameters of the scalar Higgs potential and the Higgs masses in Appendices [C] and D. In Appendix [E, we note that certain combinations of the scalar potential parameters are invariant with respect to the choice of basis for the two scalar doublets. In particular, the Higgs masses and the physical Higgs interaction vertices can be written in terms of these invariant coupling parameters. The decoupling limit of the $2 \mathrm{HDM}$ is defined in Section 3 and its main properties are examined. In this limit, the properties of the lightest CP-even Higgs boson, $h$, precisely coincide with those of the SM Higgs boson. This is shown in Section 4, where we exhibit the tree-level Higgs couplings to vector bosons, fermions and Higgs bosons, and evaluate them in the decoupling limit (cubic and quartic Higgs self-couplings are written out explicitly in Appendices $\mathrm{F}$ and $\mathrm{G}$, respectively). The first non-trivial corrections to the Higgs couplings as one moves away from the decoupling 
limit are also given. In Section 5, we note that certain parameter regimes exist outside the decoupling regime in which one of the CP-even Higgs bosons exhibits tree-level couplings that approximately coincide with those of the SM Higgs boson. We discuss the origin of this behavior and show how one can distinguish this region of parameter space from that of true decoupling. In Section 6, the two-Higgs-doublet sector of the MSSM is used to illustrate the features of the decoupling limit when $m_{A} \gg m_{Z}$. In addition, we briefly describe the impact of radiative corrections, and show how these corrections satisfy the requirements of the decoupling limit. We emphasize that the rate of approach to decoupling can be delayed at large $\tan \beta$, and we discuss the possibility of a SM-like Higgs boson in a parameter regime in which all Higgs masses are in a range $\lesssim \mathcal{O}(v)$. Finally, our conclusions are give in Section 7 .

\section{THE CP-CONSERVING TWO-HIGGS DOUBLET MODEL}

We first review the general (non-supersymmetric) two-Higgs doublet extension of the Standard Model [7]. Let $\Phi_{1}$ and $\Phi_{2}$ denote two complex $Y=1, \mathrm{SU}(2)_{L}$ doublet scalar fields. The most general gauge invariant scalar potential is given $b^{2}$

$$
\begin{aligned}
\mathcal{V}=m_{11}^{2} \Phi_{1}^{\dagger} \Phi_{1}+m_{22}^{2} \Phi_{2}^{\dagger} \Phi_{2}-\left[m_{12}^{2} \Phi_{1}^{\dagger} \Phi_{2}+\text { h.c. }\right] \\
+\frac{1}{2} \lambda_{1}\left(\Phi_{1}^{\dagger} \Phi_{1}\right)^{2}+\frac{1}{2} \lambda_{2}\left(\Phi_{2}^{\dagger} \Phi_{2}\right)^{2}+\lambda_{3}\left(\Phi_{1}^{\dagger} \Phi_{1}\right)\left(\Phi_{2}^{\dagger} \Phi_{2}\right)+\lambda_{4}\left(\Phi_{1}^{\dagger} \Phi_{2}\right)\left(\Phi_{2}^{\dagger} \Phi_{1}\right) \\
\quad+\left\{\frac{1}{2} \lambda_{5}\left(\Phi_{1}^{\dagger} \Phi_{2}\right)^{2}+\left[\lambda_{6}\left(\Phi_{1}^{\dagger} \Phi_{1}\right)+\lambda_{7}\left(\Phi_{2}^{\dagger} \Phi_{2}\right)\right] \Phi_{1}^{\dagger} \Phi_{2}+\text { h.c. }\right\}
\end{aligned}
$$

In general, $m_{12}^{2}, \lambda_{5}, \lambda_{6}$ and $\lambda_{7}$ can be complex. In many discussions of two-Higgs-doublet models, the terms proportional to $\lambda_{6}$ and $\lambda_{7}$ are absent. This can be achieved by imposing a discrete symmetry $\Phi_{1} \rightarrow-\Phi_{1}$ on the model. Such a symmetry would also require $m_{12}^{2}=0$ unless we allow a soft violation of this discrete symmetry by dimension-two terms. ${ }^{3}$ In this paper, we refrain in general from setting any of the coefficients in eq. (11) to zero.

We next derive the constraints on the parameters $\lambda_{i}$ such that the scalar potential $\mathcal{V}$ is

\footnotetext{
${ }^{2}$ In refs. 7] and [9], the scalar potential is parameterized in terms of a different set of couplings, which are less useful for the decoupling analysis. In Appendix $\mathrm{A}$ we relate this alternative set of couplings to the parameters appearing in eq. (11).

${ }^{3}$ This discrete symmetry is also employed to restrict the Higgs-fermion couplings so that no tree-level Higgs-mediated FCNC's are present. If $\lambda_{6}=\lambda_{7}=0$, but $m_{12}^{2} \neq 0$, the soft breaking of the discrete symmetry generates finite Higgs-mediated FCNC's at one loop.
} 
bounded from below. It is sufficient to examine the quartic terms of the scalar potential (which we denote by $\mathcal{V}_{4}$ ). We define $a \equiv \Phi_{1}^{\dagger} \Phi_{1}, b \equiv \Phi_{2}^{\dagger} \Phi_{2}, c \equiv \operatorname{Re} \Phi_{1}^{\dagger} \Phi_{2}, d \equiv \operatorname{Im} \Phi_{1}^{\dagger} \Phi_{2}$, and note that $a b \geq c^{2}+d^{2}$. Then, one can rewrite the quartic terms of the scalar potential as follows:

$$
\begin{aligned}
\mathcal{V}_{4}= & \frac{1}{2}\left[\lambda_{1}^{1 / 2} a-\lambda_{2}^{1 / 2} b\right]^{2}+\left[\lambda_{3}+\left(\lambda_{1} \lambda_{2}\right)^{1 / 2}\right]\left(a b-c^{2}-d^{2}\right) \\
& +2\left[\lambda_{3}+\lambda_{4}+\left(\lambda_{1} \lambda_{2}\right)^{1 / 2}\right] c^{2}+\left[\operatorname{Re} \lambda_{5}-\lambda_{3}-\lambda_{4}-\left(\lambda_{1} \lambda_{2}\right)^{1 / 2}\right]\left(c^{2}-d^{2}\right) \\
& -2 c d \operatorname{Im} \lambda_{5}+2 a\left[c \operatorname{Re} \lambda_{6}-d \operatorname{Im} \lambda_{6}\right]+2 b\left[c \operatorname{Re} \lambda_{7}-d \operatorname{Im} \lambda_{7}\right] .
\end{aligned}
$$

We demand that no directions exist in field space in which $\mathcal{V} \rightarrow-\infty$. (We also require that no flat directions exist for $\mathcal{V}_{4}$.) Three conditions on the $\lambda_{i}$ are easily obtained by examining asymptotically large values of $a$ and/or $b$ with $c=d=0$ :

$$
\lambda_{1}>0, \quad \lambda_{2}>0, \quad \lambda_{3}>-\left(\lambda_{1} \lambda_{2}\right)^{1 / 2} .
$$

A fourth condition arises by examining the direction in field space where $\lambda_{1}^{1 / 2} a=\lambda_{2}^{1 / 2} b$ and $a b=c^{2}+d^{2}$. Setting $c=\xi d$, and requiring that the potential is bounded from below for all $\xi$ leads to a condition on a quartic polynomial in $\xi$, which must be satisfied for all $\xi$. There is no simple analytical constraint on the $\lambda_{i}$ that can be derived from this condition. If $\lambda_{6}=\lambda_{7}=0$, the resulting polynomial is quadratic in $\xi$, and a constraint on the remaining nonzero $\lambda_{i}$ is easily derived [18]

$$
\left.\lambda_{3}+\lambda_{4}-\left|\lambda_{5}\right|>-\left(\lambda_{1} \lambda_{2}\right)^{1 / 2} \quad \text { [assuming } \lambda_{6}=\lambda_{7}=0\right] .
$$

In this paper, we shall ignore the possibility of explicit CP-violating effects in the Higgs potential by choosing all coefficients in eq. (II) to be real (see Appendix B). ${ }^{4}$ The scalar fields will develop non-zero vacuum expectation values if the mass matrix $m_{i j}^{2}$ has at least one negative eigenvalue. We assume that the parameters of the scalar potential are chosen such that the minimum of the scalar potential respects the $\mathrm{U}(1)_{\mathrm{EM}}$ gauge symmetry. Then, the scalar field vacuum expectations values are of the form

$$
\left\langle\Phi_{1}\right\rangle=\frac{1}{\sqrt{2}}\left(\begin{array}{c}
0 \\
v_{1}
\end{array}\right), \quad\left\langle\Phi_{2}\right\rangle=\frac{1}{\sqrt{2}}\left(\begin{array}{c}
0 \\
v_{2}
\end{array}\right),
$$

\footnotetext{
${ }^{4}$ The most general CP-violating 2HDM will be examined in ref. 14].
} 
where the $v_{i}$ are taken to be real, i.e. we assume that spontaneous $\mathrm{CP}$ violation does not occur. ${ }^{5}$ The corresponding potential minimum conditions are:

$$
\begin{aligned}
& m_{11}^{2}=m_{12}^{2} t_{\beta}-\frac{1}{2} v^{2}\left[\lambda_{1} c_{\beta}^{2}+\lambda_{345} s_{\beta}^{2}+3 \lambda_{6} s_{\beta} c_{\beta}+\lambda_{7} s_{\beta}^{2} t_{\beta}\right], \\
& m_{22}^{2}=m_{12}^{2} t_{\beta}^{-1}-\frac{1}{2} v^{2}\left[\lambda_{2} s_{\beta}^{2}+\lambda_{345} c_{\beta}^{2}+\lambda_{6} c_{\beta}^{2} t_{\beta}^{-1}+3 \lambda_{7} s_{\beta} c_{\beta}\right],
\end{aligned}
$$

where we have defined:

$$
\lambda_{345} \equiv \lambda_{3}+\lambda_{4}+\lambda_{5}, \quad t_{\beta} \equiv \tan \beta \equiv \frac{v_{2}}{v_{1}},
$$

and

$$
v^{2} \equiv v_{1}^{2}+v_{2}^{2}=\frac{4 m_{W}^{2}}{g^{2}}=(246 \mathrm{GeV})^{2} .
$$

It is always possible to choose the phases of the scalar doublet Higgs fields such that both $v_{1}$ and $v_{2}$ are positive; henceforth we take $0 \leq \beta \leq \pi / 2$.

Of the original eight scalar degrees of freedom, three Goldstone bosons $\left(G^{ \pm}\right.$and $\left.G\right)$ are absorbed ("eaten") by the $W^{ \pm}$and $Z$. The remaining five physical Higgs particles are: two CP-even scalars ( $h$ and $H$, with $m_{h} \leq m_{H}$ ), one CP-odd scalar $(A)$ and a charged Higgs pair $\left(H^{ \pm}\right)$. The squared-mass parameters $m_{11}^{2}$ and $m_{22}^{2}$ can be eliminated by minimizing the scalar potential. The resulting squared-masses for the CP-odd and charged Higgs states $\operatorname{are}^{6}$

$$
\begin{aligned}
m_{A}^{2} & =\frac{m_{12}^{2}}{s_{\beta} c_{\beta}}-\frac{1}{2} v^{2}\left(2 \lambda_{5}+\lambda_{6} t_{\beta}^{-1}+\lambda_{7} t_{\beta}\right), \\
m_{H^{ \pm}}^{2} & =m_{A^{0}}^{2}+\frac{1}{2} v^{2}\left(\lambda_{5}-\lambda_{4}\right) .
\end{aligned}
$$

The two CP-even Higgs states mix according to the following squared-mass matrix:

$$
\mathcal{M}^{2} \equiv m_{A^{0}}^{2}\left(\begin{array}{cc}
s_{\beta}^{2} & -s_{\beta} c_{\beta} \\
-s_{\beta} c_{\beta} & c_{\beta}^{2}
\end{array}\right)+\mathcal{B}^{2}
$$

where

$$
\mathcal{B}^{2} \equiv v^{2}\left(\begin{array}{cc}
\lambda_{1} c_{\beta}^{2}+2 \lambda_{6} s_{\beta} c_{\beta}+\lambda_{5} s_{\beta}^{2} & \left(\lambda_{3}+\lambda_{4}\right) s_{\beta} c_{\beta}+\lambda_{6} c_{\beta}^{2}+\lambda_{7} s_{\beta}^{2} \\
\left(\lambda_{3}+\lambda_{4}\right) s_{\beta} c_{\beta}+\lambda_{6} c_{\beta}^{2}+\lambda_{7} s_{\beta}^{2} & \lambda_{2} s_{\beta}^{2}+2 \lambda_{7} s_{\beta} c_{\beta}+\lambda_{5} c_{\beta}^{2}
\end{array}\right)
$$

\footnotetext{
${ }^{5}$ The conditions required for the absence of explicit and spontaneous CP-violation in the Higgs sector are elucidated in Appendix B

${ }^{6}$ Here and in the following, we use the shorthand notation $c_{\beta} \equiv \cos \beta, s_{\beta} \equiv \sin \beta, c_{\alpha} \equiv \cos \alpha, s_{\alpha} \equiv \sin \alpha$, $c_{2 \alpha} \equiv \cos 2 \alpha, s_{2 \alpha} \equiv \cos 2 \alpha, c_{\beta-\alpha} \equiv \cos (\beta-\alpha), s_{\beta-\alpha} \equiv \sin (\beta-\alpha)$, etc.
} 
Defining the physical mass eigenstates

$$
\begin{aligned}
H & =\left(\sqrt{2} \operatorname{Re} \Phi_{1}^{0}-v_{1}\right) c_{\alpha}+\left(\sqrt{2} \operatorname{Re} \Phi_{2}^{0}-v_{2}\right) s_{\alpha}, \\
h & =-\left(\sqrt{2} \operatorname{Re} \Phi_{1}^{0}-v_{1}\right) s_{\alpha}+\left(\sqrt{2} \operatorname{Re} \Phi_{2}^{0}-v_{2}\right) c_{\alpha},
\end{aligned}
$$

the masses and mixing angle $\alpha$ are found from the diagonalization process

$$
\begin{aligned}
& \left(\begin{array}{cc}
m_{H}^{2} & 0 \\
0 & m_{h}^{2}
\end{array}\right)=\left(\begin{array}{cc}
c_{\alpha} & s_{\alpha} \\
-s_{\alpha} & c_{\alpha}
\end{array}\right)\left(\begin{array}{cc}
\mathcal{M}_{11}^{2} & \mathcal{M}_{12}^{2} \\
\mathcal{M}_{12}^{2} & \mathcal{M}_{22}^{2}
\end{array}\right)\left(\begin{array}{cc}
c_{\alpha} & -s_{\alpha} \\
s_{\alpha} & c_{\alpha}
\end{array}\right) \\
& \quad=\left(\begin{array}{cc}
\mathcal{M}_{11}^{2} c_{\alpha}^{2}+2 \mathcal{M}_{12}^{2} c_{\alpha} s_{\alpha}+\mathcal{M}_{22}^{2} s_{\alpha}^{2} & \mathcal{M}_{12}^{2}\left(c_{\alpha}^{2}-s_{\alpha}^{2}\right)+\left(\mathcal{M}_{22}^{2}-\mathcal{M}_{11}^{2}\right) s_{\alpha} c_{\alpha} \\
\mathcal{M}_{12}^{2}\left(c_{\alpha}^{2}-s_{\alpha}^{2}\right)+\left(\mathcal{M}_{22}^{2}-\mathcal{M}_{11}^{2}\right) s_{\alpha} c_{\alpha} & \mathcal{M}_{11}^{2} s_{\alpha}^{2}-2 \mathcal{M}_{12}^{2} c_{\alpha} s_{\alpha}+\mathcal{M}_{22}^{2} c_{\alpha}^{2}
\end{array}\right) .
\end{aligned}
$$

The mixing angle $\alpha$ is evaluated by setting the off-diagonal elements of the CP-even scalar squared-mass matrix [eq. (15)] to zero, and demanding that $m_{H} \geq m_{h}$. The end result is

$$
m_{H, h}^{2}=\frac{1}{2}\left[\mathcal{M}_{11}^{2}+\mathcal{M}_{22}^{2} \pm \sqrt{\left(\mathcal{M}_{11}^{2}-\mathcal{M}_{22}^{2}\right)^{2}+4\left(\mathcal{M}_{12}^{2}\right)^{2}}\right]
$$

and the corresponding CP-even scalar mixing angle is fixed by

$$
\begin{aligned}
& s_{2 \alpha}=\frac{2 \mathcal{M}_{12}^{2}}{\sqrt{\left(\mathcal{M}_{11}^{2}-\mathcal{M}_{22}^{2}\right)^{2}+4\left(\mathcal{M}_{12}^{2}\right)^{2}}}, \\
& c_{2 \alpha}=\frac{\mathcal{M}_{11}^{2}-\mathcal{M}_{22}^{2}}{\sqrt{\left(\mathcal{M}_{11}^{2}-\mathcal{M}_{22}^{2}\right)^{2}+4\left(\mathcal{M}_{12}^{2}\right)^{2}}} .
\end{aligned}
$$

We shall take $-\pi / 2 \leq \alpha \leq \pi / 2$.

It is convenient to define the following four combinations of parameters:

$$
\begin{aligned}
m_{D}^{4} & \equiv \mathcal{B}_{11}^{2} \mathcal{B}_{22}^{2}-\left[\mathcal{B}_{12}^{2}\right]^{2}, \\
m_{L}^{2} & \equiv \mathcal{B}_{11}^{2} \cos ^{2} \beta+\mathcal{B}_{22}^{2} \sin ^{2} \beta+\mathcal{B}_{12}^{2} \sin 2 \beta \\
m_{T}^{2} & \equiv \mathcal{B}_{11}^{2}+\mathcal{B}_{22}^{2}, \\
m_{S}^{2} & \equiv m_{A}^{2}+m_{T}^{2},
\end{aligned}
$$

where the $\mathcal{B}_{i j}^{2}$ are the elements of the matrix defined in eq. (13). In terms of these quantities we have the exact relations

$$
m_{H, h}^{2}=\frac{1}{2}\left[m_{S}^{2} \pm \sqrt{m_{S}^{4}-4 m_{A}^{2} m_{L}^{2}-4 m_{D}^{4}}\right]
$$


and

$$
c_{\beta-\alpha}^{2}=\frac{m_{L}^{2}-m_{h}^{2}}{m_{H}^{2}-m_{h}^{2}} .
$$

Eq. (201) is most easily derived by using $c_{\beta-\alpha}^{2}=\frac{1}{2}\left(1+c_{2 \beta} c_{2 \alpha}+s_{2 \beta} s_{2 \alpha}\right)$ and the results of eq. (17). Note that the case of $m_{h}=m_{H}$ is special and must be treated carefully. We do this in Appendix $\mathbb{C}$, where we explicitly verify that $0 \leq c_{\beta-\alpha}^{2} \leq 1$.

Finally, for completeness we record the expressions for the original hypercharge-one scalar fields $\Phi_{i}$ in terms of the physical Higgs states and the Goldstone bosons:

$$
\begin{aligned}
\Phi_{1}^{ \pm} & =c_{\beta} G^{ \pm}-s_{\beta} H^{ \pm}, \\
\Phi_{2}^{ \pm} & =s_{\beta} G^{ \pm}+c_{\beta} H^{ \pm}, \\
\Phi_{1}^{0} & =\frac{1}{\sqrt{2}}\left[v_{1}+c_{\alpha} H-s_{\alpha} h+i c_{\beta} G-i s_{\beta} A\right], \\
\Phi_{2}^{0} & =\frac{1}{\sqrt{2}}\left[v_{2}+s_{\alpha} H+c_{\alpha} h+i s_{\beta} G+i c_{\beta} A\right] .
\end{aligned}
$$

\section{THE DECOUPLING LIMIT}

In effective field theory, we may examine the behavior of the theory characterized by two disparate mass scales, $m_{L} \ll m_{S}$, by integrating out all particles with masses of order $m_{S}$, assuming that all the couplings of the "low-mass" effective theory comprising particles with masses of order $m_{L}$ can be kept fixed. In the 2HDM, the low-mass effective theory, if it exists, must correspond to the case where one of the Higgs doublets is integrated out. That is, the resulting effective low-mass theory is precisely equivalent to the one-scalar-doublet SM Higgs sector. These conclusions follow from electroweak gauge invariance. Namely, there are two relevant scales - the electroweak scale characterized by the scale $v=246 \mathrm{GeV}$ and a second scale $m_{S} \gg v$. The underlying electroweak symmetry requires that scalar mass splittings within doublets cannot be larger than $\mathcal{O}(v)$ [assuming that dimensionless couplings of the theory are no larger than $\mathcal{O}(1)]$. It follows that the $H^{ \pm}, A$ and $H$ masses must be of $\mathcal{O}\left(m_{S}\right)$, while $m_{h} \sim \mathcal{O}(v)$. Moreover, since the effective low-mass theory consists of a one-doublet Higgs sector, the properties of $h$ must be indistinguishable from those of the SM Higgs boson.

We can illustrate these results more explicitly as follows. Suppose that all the Higgs self-coupling constants $\lambda_{i}$ are held fixed such that $\left|\lambda_{i}\right| \lesssim \mathcal{O}(1)$, while taking $m_{A}^{2} \gg\left|\lambda_{i}\right| v^{2}$. In particular, we constrain the $\alpha_{i} \equiv \lambda_{i} /(4 \pi)$ so that the Higgs sector does not become strongly 
coupled, implying no violations of tree-unitarity [19, 20, 21, 22, 23]. Then, the $\mathcal{B}_{i j}^{2} \sim \mathcal{O}\left(v^{2}\right)$, and it follows that:

$$
\begin{aligned}
m_{h} & \simeq m_{L}=\mathcal{O}(v) \\
m_{H}, m_{A}, m_{H^{ \pm}} & =m_{S}+\mathcal{O}\left(v^{2} / m_{S}\right)
\end{aligned}
$$

and

$$
\begin{aligned}
\cos ^{2}(\beta-\alpha) & \simeq \frac{m_{L}^{2}\left(m_{T}^{2}-m_{L}^{2}\right)-m_{D}^{4}}{m_{A}^{4}} \\
& =\frac{\left[\frac{1}{2}\left(\mathcal{B}_{11}^{2}-\mathcal{B}_{22}^{2}\right) s_{2 \beta}-\mathcal{B}_{12}^{2} c_{2 \beta}\right]^{2}}{m_{A}^{4}}=\mathcal{O}\left(\frac{v^{4}}{m_{S}^{4}}\right) .
\end{aligned}
$$

We shall establish the above results in more detail below.

The limit $m_{A}^{2} \gg\left|\lambda_{i}\right| v^{2}$ (subject to $\left|\alpha_{i}\right| \lesssim 1$ ) is called the decoupling limit of the model. ${ }^{7}$ Note that eq. (24) implies that in the decoupling limit, $c_{\beta-\alpha}=\mathcal{O}\left(v^{2} / m_{A}^{2}\right)$. We will demonstrate that this implies that the couplings of $h$ in the decoupling limit approach values that correspond precisely to those of the SM Higgs boson. We will also obtain explicit expressions for the squared-mass differences between the heavy Higgs bosons (as a function of the $\lambda_{i}$ couplings in the Higgs potential) in the decoupling limit.

One can give an alternative condition for the decoupling limit. As above, we assume that all $\left|\alpha_{i}\right| \lesssim 1$. First consider the following special cases. If neither $\tan \beta$ nor $\cot \beta$ is close to 0 , then $m_{12}^{2} \gg\left|\lambda_{i}\right| v^{2}$ [see eq. (10)] in the decoupling limit. On the other hand, if $m_{12}^{2} \sim \mathcal{O}\left(v^{2}\right)$ and $\tan \beta \gg 1[\cot \beta \gg 1]$, then it follows from eqs. (6) and (7) that $m_{11}^{2} \gg \mathcal{O}\left(v^{2}\right)$ if $\lambda_{7}<0\left[m_{22}^{2} \gg \mathcal{O}\left(v^{2}\right)\right.$ if $\left.\lambda_{6}<0\right]$ in the decoupling limit. All such conditions depend on the original choice of scalar field basis $\Phi_{1}$ and $\Phi_{2}$. For example, we can diagonalize the squared-mass terms of the scalar potential [eq. (11)] thereby setting $m_{12}=0$. In the decoupling limit in the new basis, one is simply driven to the second case above. A basisindependent characterization of the decoupling limit is simple to formulate. Starting from the scalar potential in an arbitrary basis, form the matrix $m_{i j}^{2}$ [made up of the coefficients of the quadratic terms in the potential, see eq. (10)]. Denote the eigenvalues of this matrix by $m_{a}^{2}$ and $m_{b}^{2}$ respectively; note that the eigenvalues are real but can be of either sign. By

\footnotetext{
${ }^{7}$ In Section 4 [see eq. (51) and surrounding discussion], we shall refine this definition slightly, and also require that $m_{A}^{2} \gg\left|\lambda_{6}\right| v^{2} \cot \beta$ and $m_{A}^{2} \gg\left|\lambda_{7}\right| v^{2} \tan \beta$, in order to guarantee that at large $\cot \beta[\tan \beta]$ the couplings of $h$ to up-type [down-type] fermions approach the corresponding SM Higgs-fermion couplings.
} 
convention, we can take $\left|m_{a}^{2}\right| \leq\left|m_{b}^{2}\right|$. Then, the decoupling limit corresponds to $m_{a}^{2}<0$, $m_{b}^{2}>0$ such that $m_{b}^{2} \gg\left|m_{a}^{2}\right|, v^{2}$ (with $\left|\alpha_{i}\right| \lesssim 1$ ).

For some choices of the scalar potential, no decoupling limit exists. Consider the case of $m_{12}^{2}=\lambda_{6}=\lambda_{7}=0$ (and all other $\left|\alpha_{i}\right| \lesssim 1$ ). Then, the potential minimum conditions [eqs. (6) and (7)] do not permit either $m_{11}^{2}$ or $m_{22}^{2}$ to become large; $m_{11}^{2}, m_{22}^{2} \sim \mathcal{O}\left(v^{2}\right)$, and clearly all Higgs masses are of $\mathcal{O}(v)$. Thus, in this case no decoupling limit exists. ${ }^{8}$ The case of $m_{12}^{2}=\lambda_{6}=\lambda_{7}=0$ corresponds to the existence of a discrete symmetry in which the potential is invariant under the change of sign of one of the Higgs doublet fields. Although the latter statement is basis-dependent, one can check that the following stronger condition holds: no decoupling limit exists if and only if $\lambda_{6}=\lambda_{7}=0$ in the basis where $m_{12}^{2}=0$. Thus, the absence of a decoupling limit implies the existence of some discrete symmetry under which the scalar potential is invariant (although the precise form of this symmetry is most evident for the special choice of basis).

We now return to the results for the Higgs masses and the CP-even Higgs mixing angle in the decoupling limit. For fixed values of $\lambda_{6}, \lambda_{7}, \alpha$ and $\beta$, there are two equivalent parameter sets: (i) $\lambda_{1}, \lambda_{2}, \lambda_{3}, \lambda_{4}$ and $\lambda_{5}$; (ii) $m_{h}^{2}, m_{H}^{2}, m_{12}^{2}, m_{H^{ \pm}}^{2}$ and $m_{A}^{2}$. The relations between these two parameter sets are given in Appendix D, Using the results eqs. (D3)-(D7) we can give explicit expressions in the decoupling limit for the Higgs masses in terms of the potential parameters and the mixing angles. First, it is convenient to define the following four linear combinations of the $\lambda_{i}:^{9}$

$$
\begin{aligned}
\lambda & \equiv \lambda_{1} c_{\beta}^{4}+\lambda_{2} s_{\beta}^{4}+\frac{1}{2} \lambda_{345} s_{2 \beta}^{2}+2 s_{2 \beta}\left(\lambda_{6} c_{\beta}^{2}+\lambda_{7} s_{\beta}^{2}\right), \\
\hat{\lambda} & \equiv \frac{1}{2} s_{2 \beta}\left[\lambda_{1} c_{\beta}^{2}-\lambda_{2} s_{\beta}^{2}-\lambda_{345} c_{2 \beta}\right]-\lambda_{6} c_{\beta} c_{3 \beta}-\lambda_{7} s_{\beta} s_{3 \beta}, \\
\lambda_{A} & \equiv c_{2 \beta}\left(\lambda_{1} c_{\beta}^{2}-\lambda_{2} s_{\beta}^{2}\right)+\lambda_{345} s_{2 \beta}^{2}-\lambda_{5}+2 \lambda_{6} c_{\beta} s_{3 \beta}-2 \lambda_{7} s_{\beta} c_{3 \beta}, \\
\lambda_{F} & \equiv \lambda_{5}-\lambda_{4},
\end{aligned}
$$

where $\lambda_{345}$ is defined in eq. (8). The significance of these coupling combinations is discussed in Appendix E We consider the limit $c_{\beta-\alpha} \rightarrow 0$, corresponding to the decoupling limit, $m_{A}^{2} \gg\left|\lambda_{i}\right| v^{2}$. In nearly all of the parameter space, $\mathcal{M}_{12}^{2}<0$ [see eq. (12)], and it follows

\footnotetext{
${ }^{8}$ However, it may be difficult to distinguish between the non-decoupling effects of the SM with a heavy Higgs boson and those of the 2HDM where all Higgs bosons are heavy [24].

${ }^{9}$ We make use of the triple-angle identities: $c_{3 \beta}=c_{\beta}\left(c_{\beta}^{2}-3 s_{\beta}^{2}\right)$ and $s_{3 \beta}=s_{\beta}\left(3 c_{\beta}^{2}-s_{\beta}^{2}\right)$.
} 
from eq. (17) that $-\pi / 2 \leq \alpha \leq 0$ (which implies that $c_{\beta-\alpha} \rightarrow 0$ is equivalent to $\beta-\alpha \rightarrow \pi / 2$ given that $0 \leq \beta \leq \pi / 2)$. However, in the small regions of parameter space in which $\beta$ is near zero [or $\pi / 2$ ], roughly corresponding to $m_{A}^{2} \tan \beta<\lambda_{6} v^{2}$ [or $m_{A}^{2} \cot \beta<\lambda_{7} v^{2}$ ], one finds $\mathcal{M}_{12}^{2}>0$ (and consequently $0<\alpha<\pi / 2$ ). In these last two cases, the decoupling limit is achieved for $\alpha=\pi / 2-\beta$ and $\cot \beta \gg 1[\tan \beta \gg 1]$. That is, $\cos (\beta-\alpha)=\sin 2 \beta \ll 1$ and $\sin (\beta-\alpha) \simeq-1[+1] .{ }^{10}$ In practice, since $\tan \beta$ is fixed and cannot be arbitrarily large (or arbitrarily close to zero), one can always find a value of $m_{A}$ large enough such that $\mathcal{M}_{12}^{2}<0$. This is equivalent to employing the refined version of the decoupling limit mentioned in footnote 7 . In this case, the decoupling limit simply corresponds to $\beta-\alpha \rightarrow \pi / 2$ [i.e., $\sin (\beta-\alpha)=1]$ independently of the value of $\beta$.

In the approach to the decoupling limit where $\alpha \simeq \beta-\pi / 2$ (that is, $\left|c_{\beta-\alpha}\right| \ll 1$ and $s_{\beta-\alpha} \simeq 1-\frac{1}{2} c_{\beta-\alpha}^{2}$ ), we may use eqs. (D91)-(D12) and eq. (11) to obtain: ${ }^{11}$

$$
\begin{aligned}
m_{A}^{2} & \simeq v^{2}\left[\frac{\hat{\lambda}}{c_{\beta-\alpha}}+\lambda_{A}-\frac{3}{2} \widehat{\lambda} c_{\beta-\alpha}\right] \\
m_{h}^{2} & \simeq v^{2}\left(\lambda-\hat{\lambda} c_{\beta-\alpha}\right) \\
m_{H}^{2} & \simeq v^{2}\left[\frac{\hat{\lambda}}{c_{\beta-\alpha}}+\lambda-\frac{1}{2} \widehat{\lambda} c_{\beta-\alpha}\right] \simeq m_{A}^{2}+\left(\lambda-\lambda_{A}+\hat{\lambda} c_{\beta-\alpha}\right) v^{2} \\
m_{H^{ \pm}}^{2} & \simeq v^{2}\left[\frac{\hat{\lambda}}{c_{\beta-\alpha}}+\lambda_{A}+\frac{1}{2} \lambda_{F}-\frac{3}{2} \widehat{\lambda} c_{\beta-\alpha}\right]=m_{A}^{2}+\frac{1}{2} \lambda_{F} v^{2} .
\end{aligned}
$$

The condition $m_{H}>m_{h}$ implies the inequality (valid to first order in $c_{\beta-\alpha}$ ):

$$
m_{A}^{2}>v^{2}\left(\lambda_{A}-2 \widehat{\lambda} c_{\beta-\alpha}\right)
$$

[cf. eq. (D32)]. The positivity of $m_{h}^{2}$ also imposes a useful constraint on the Higgs potential parameters. For example, $m_{h}^{2}>0$ requires that $\lambda>0$.

In the decoupling limit (where $m_{A}^{2} \gg\left|\lambda_{i}\right| v^{2}$ ), eqs. (29)-(32) provide the first nontrivial corrections to eqs. (22) and (23). Finally, we employ eq. (10) to obtain

$$
m_{12}^{2} \simeq v^{2} s_{\beta} c_{\beta}\left[\frac{\hat{\lambda}}{c_{\beta-\alpha}}+\lambda_{A}+\lambda_{5}+\frac{1}{2} \lambda_{6} t_{\beta}^{-1}+\frac{1}{2} \lambda_{7} t_{\beta}-\frac{3}{2} \widehat{\lambda} c_{\beta-\alpha}\right] \text {. }
$$

${ }^{10}$ We have chosen a convention in which $-\pi / 2 \leq \alpha \leq \pi / 2$. An equally good alternative is to choose $\sin (\beta-\alpha) \geq 0$. If negative, one may simply change the sign of $\sin (\beta-\alpha)$ by taking $\alpha \rightarrow \alpha \pm \pi$, which is equivalent to the field redefinitions $h \rightarrow-h, H \rightarrow-H$.

${ }^{11}$ In obtaining eqs. (29), (31) and (32) we divided both sides of each equation by $c_{\beta-\alpha}$, so these equations need to be treated with care if $c_{\beta-\alpha}=0$ exactly. In this latter case, it suffices to note that $\hat{\lambda} / c_{\beta-\alpha}$ has a finite limit whose value depends on $m_{A}$ and $\lambda_{A}$ [see eq. (36)] . 
This result confirms our previous observation that $m_{12}^{2} \gg\left|\lambda_{i}\right| v^{2}$ in the decoupling limit as long as $\beta$ is not close to 0 or $\pi / 2$. However, $m_{12}^{2}$ can be of $\mathcal{O}\left(v^{2}\right)$ in the decoupling limit $\left[c_{\beta-\alpha} \rightarrow 0\right.$ ] if either $t_{\beta} \gg 1$ [and $\left.c_{\beta} / c_{\beta-\alpha} \sim \mathcal{O}(1)\right]$ or $t_{\beta}^{-1} \gg 1\left[\right.$ and $\left.s_{\beta} / c_{\beta-\alpha} \sim \mathcal{O}(1)\right]$.

The significance of eq. (30) is easily understood by noting that the decoupling limit corresponds to integrating out the second heavy Higgs doublet. The resulting low-mass effective theory is simply the one-Higgs-doublet model with corresponding scalar potential $V=m^{2}\left(\Phi^{\dagger} \Phi\right)+\frac{1}{2} \lambda\left(\Phi^{\dagger} \Phi\right)^{2}$, where $\lambda$ is given by eq. (25) and

$$
m^{2} \equiv m_{11}^{2} c_{\beta}^{2}+m_{22}^{2} s_{\beta}^{2}-2 m_{12}^{2} s_{\beta} c_{\beta}
$$

Imposing the potential minimum conditions [eqs. (6) and (17)], we see that $v^{2}=-2 m^{2} / \lambda$ [where $\left.\left\langle\Phi^{0}\right\rangle \equiv v / \sqrt{2}\right]$ as expected. Moreover, the Higgs mass is given by $m_{h}^{2}=\lambda v^{2}$, in agreement with the $c_{\beta-\alpha} \rightarrow 0$ limit of eq. (30).

We can rewrite eq. (29) in another form [or equivalently use eqs. (D30) and (D31) to obtain]:

$$
\cos (\beta-\alpha) \simeq \frac{\widehat{\lambda} v^{2}}{m_{A}^{2}-\lambda_{A} v^{2}} \simeq \frac{\widehat{\lambda} v^{2}}{m_{H}^{2}-m_{h}^{2}} .
$$

This yields an $\mathcal{O}\left(v^{2} / m_{A}^{2}\right)$ correction to eq. (24). Note that eq. (36) also implies that in the approach to the decoupling limit, the sign of $\cos (\beta-\alpha)$ is given by the sign of $\hat{\lambda}$.

\section{TWO-HIGGS DOUBLET MODEL COUPLINGS IN THE DECOUPLING LIMIT}

The phenomenology of the two-Higgs doublet model depends in detail on the various couplings of the Higgs bosons to gauge bosons, Higgs bosons and fermions 7]. The Higgs couplings to gauge bosons follow from gauge invariance and are thus model independent:

$$
g_{h V V}=g_{V} m_{V} s_{\beta-\alpha}, \quad g_{H V V}=g_{V} m_{V} c_{\beta-\alpha},
$$

where $g_{V} \equiv 2 m_{V} / v$ for $V=W$ or $Z$. There are no tree-level couplings of $A$ or $H^{ \pm}$to $V V$. In the decoupling limit where $c_{\beta-\alpha}=0$, we see that $g_{h V V}=g_{h_{\mathrm{SM} V} V}$, whereas the $H V V$ coupling vanishes. Gauge invariance also determines the strength of the trilinear couplings of one gauge boson to two Higgs bosons:

$$
g_{h A Z}=\frac{g c_{\beta-\alpha}}{2 \cos \theta_{W}}, \quad g_{H A Z}=\frac{-g s_{\beta-\alpha}}{2 \cos \theta_{W}} .
$$


In the decoupling limit, the $h A Z$ coupling vanishes, while the $H A Z$ coupling attains its maximal value. This pattern is repeated in all the three-point and four-point couplings of $h$ and $H$ to $V V, V \phi$, and $V V \phi$ final states (where $V$ is a vector boson and $\phi$ is one of the Higgs scalars). These results can be summarized as follows: the coupling of $h$ and $H$ to vector boson pairs or vector-scalar boson final states is proportional to either $\sin (\beta-\alpha)$ or $\cos (\beta-\alpha)$ as indicated below [7, 9].

$\begin{array}{ll}\frac{\cos (\beta-\alpha)}{H W^{+} W^{-}} & \frac{\sin (\beta-\alpha)}{h W^{+} W^{-}} \\ H Z Z & h Z Z \\ Z A h & Z A H \\ W^{ \pm} H^{\mp} h & W^{ \pm} H^{\mp} H \\ Z W^{ \pm} H^{\mp} h & Z W^{ \pm} H^{\mp} H \\ \gamma W^{ \pm} H^{\mp} h & \gamma W^{ \pm} H^{\mp} H\end{array}$

Note in particular that all vertices in the theory that contain at least one vector boson and exactly one of the non-minimal Higgs boson states $\left(H, A\right.$ or $\left.H^{ \pm}\right)$are proportional to $\cos (\beta-\alpha)$ and hence vanish in the decoupling limit.

The Higgs couplings to fermions are model dependent. The most general structure for the Higgs-fermion Yukawa couplings, often referred to as the type-III model [17], is given by:

$$
-\mathcal{L}_{Y}=\bar{Q}_{L}^{0} \widetilde{\Phi}_{1} \eta_{1}^{U, 0} U_{R}^{0}+\bar{Q}_{L}^{0} \Phi_{1} \eta_{1}^{D, 0} D_{R}^{0}+\bar{Q}_{L}^{0} \widetilde{\Phi}_{2} \eta_{2}^{U, 0} U_{R}^{0}+\bar{Q}_{L}^{0} \Phi_{2} \eta_{2}^{D, 0} D_{R}^{0}+\text { h.c. }
$$

where $\Phi_{1,2}$ are the Higgs doublets, $\widetilde{\Phi}_{i} \equiv i \sigma_{2} \Phi_{i}^{*}, Q_{L}^{0}$ is the weak isospin quark doublet, and $U_{R}^{0}, D_{R}^{0}$ are weak isospin quark singlets. [The right and left-handed fermion fields are defined as usual: $\psi_{R, L} \equiv P_{R, L} \psi$, where $P_{R, L} \equiv \frac{1}{2}\left(1 \pm \gamma_{5}\right)$.] Here, $Q_{L}^{0}, U_{R}^{0}, D_{R}^{0}$ denote the interaction basis states, which are vectors in flavor space, whereas $\eta_{1}^{U, 0}, \eta_{2}^{U, 0}, \eta_{1}^{D, 0}, \eta_{2}^{D, 0}$ are matrices in flavor space. We have omitted the leptonic couplings in eq. (40); these follow the same pattern as the down-type quark couplings.

We next shift the scalar fields according to their vacuum expectation values, and then reexpress the scalars in terms of the physical Higgs states and Goldstone bosons [see eq. (21)]. In addition, we diagonalize the quark mass matrices and define the quark mass eigenstates. The resulting Higgs-fermion Lagrangian can be written in several ways [25]. We choose to display the form that makes the type-II model limit of the general type-III couplings 
apparent. The type-II model (where $\eta_{1}^{U, 0}=\eta_{2}^{D, 0}=0$ ) automatically has no tree-level flavorchanging neutral Higgs couplings, whereas these are generally present for type-III couplings. The fermion mass eigenstates are related to the interaction eigenstates by biunitary transformations:

$$
\begin{array}{ll}
P_{L} U=V_{L}^{U} P_{L} U^{0}, & P_{R} U=V_{R}^{U} P_{R} U^{0}, \\
P_{L} D=V_{L}^{D} P_{L} D^{0}, & P_{R} D=V_{R}^{D} P_{R} D^{0},
\end{array}
$$

and the Cabibbo-Kobayashi-Maskawa matrix is defined as $K \equiv V_{L}^{U} V_{L}^{D \dagger}$. It is also convenient to define "rotated" coupling matrices:

$$
\eta_{i}^{U} \equiv V_{L}^{U} \eta_{i}^{U, 0} V_{R}^{U \dagger}, \quad \eta_{i}^{D} \equiv V_{L}^{D} \eta_{i}^{D, 0} V_{R}^{D \dagger}
$$

The diagonal quark mass matrices are obtained by replacing the scalar fields with their vacuum expectation values:

$$
M_{D}=\frac{1}{\sqrt{2}}\left(v_{1} \eta_{1}^{D}+v_{2} \eta_{2}^{D}\right), \quad M_{U}=\frac{1}{\sqrt{2}}\left(v_{1} \eta_{1}^{U}+v_{2} \eta_{2}^{U}\right)
$$

After eliminating $\eta_{2}^{U}$ and $\eta_{1}^{D}$, the resulting Yukawa couplings are:

$$
\begin{aligned}
\mathcal{L}_{Y}= & \frac{1}{v} \bar{D} M_{D} D\left(\frac{s_{\alpha}}{c_{\beta}} h-\frac{c_{\alpha}}{c_{\beta}} H\right)+\frac{i}{v} \bar{D} M_{D} \gamma_{5} D\left(t_{\beta} A-G\right) \\
& -\frac{1}{\sqrt{2} c_{\beta}} \bar{D}\left(\eta_{2}^{D} P_{R}+\eta_{2}^{D^{\dagger}} P_{L}\right) D\left(c_{\beta-\alpha} h-s_{\beta-\alpha} H\right)-\frac{i}{\sqrt{2} c_{\beta}} \bar{D}\left(\eta_{2}^{D} P_{R}-\eta_{2}^{D^{\dagger}} P_{L}\right) D A \\
& -\frac{1}{v} \bar{U} M_{U} U\left(\frac{c_{\alpha}}{s_{\beta}} h+\frac{s_{\alpha}}{s_{\beta}} H\right)+\frac{i}{v} \bar{U} M_{U} \gamma_{5} U\left(t_{\beta}^{-1} A+G\right) \\
& +\frac{1}{\sqrt{2} s_{\beta}} \bar{U}\left(\eta_{1}^{U} P_{R}+\eta_{1}^{U^{\dagger}} P_{L}\right) U\left(c_{\beta-\alpha} h-s_{\beta-\alpha} H\right)-\frac{i}{\sqrt{2} s_{\beta}} \bar{U}\left(\eta_{1}^{U} P_{R}-\eta_{1}^{U^{\dagger}} P_{L}\right) U A \\
& +\frac{\sqrt{2}}{v}\left[\bar{U} K M_{D} P_{R} D\left(t_{\beta} H^{+}-G^{+}\right)+\bar{U} M_{U} K P_{L} D\left(t_{\beta}^{-1} H^{+}+G^{+}\right)+\text {h.c. }\right] \\
& -\left[\frac{1}{s_{\beta}} \bar{U} \eta_{1}^{U \dagger} K P_{L} D H^{+}+\frac{1}{c_{\beta}} \bar{U} K \eta_{2}^{D} P_{R} D H^{+}+\text {h.c. }\right] .
\end{aligned}
$$

In general, $\eta_{1}^{U}$ and $\eta_{2}^{D}$ are complex non-diagonal matrices. Thus, the Yukawa Lagrangian displayed in eq. (44) exhibits both flavor-nondiagonal and CP-violating couplings between the neutral Higgs bosons and the quarks.

In the decoupling limit (where $c_{\beta-\alpha} \rightarrow 0$ ), the Yukawa Lagrangian displays a number of interesting features. First, the flavor non-diagonal and the CP-violating couplings of $h$ vanish (although the corresponding couplings to $H$ and $A$ persist). Moreover, in this limit, the $h$ 
coupling to fermions reduces precisely to its Standard Model value, $\mathcal{L}_{Y}^{\mathrm{SM}}=-\left(m_{f} / v\right) \bar{f} f h$. To better see the behavior of couplings in the decoupling limit, the following trigonometric identities are particularly useful:

$$
\begin{aligned}
h \bar{D} D: & -\frac{\sin \alpha}{\cos \beta}=\sin (\beta-\alpha)-\tan \beta \cos (\beta-\alpha), \\
h \bar{U} U: & \frac{\cos \alpha}{\sin \beta}=\sin (\beta-\alpha)+\cot \beta \cos (\beta-\alpha), \\
H \bar{D} D: & \frac{\cos \alpha}{\cos \beta}=\cos (\beta-\alpha)+\tan \beta \sin (\beta-\alpha), \\
H \bar{U} U: & \frac{\sin \alpha}{\sin \beta}=\cos (\beta-\alpha)-\cot \beta \sin (\beta-\alpha),
\end{aligned}
$$

where we have indicated the type of Higgs-fermion coupling with which a particular trigonometric expression arises. It is now easy to read off the corresponding Higgs-fermion couplings in the decoupling limit and one verifies that the $h$-fermion couplings reduce to their Standard Model values. Working to $\mathcal{O}\left(c_{\beta-\alpha}\right)$, the Yukawa couplings of $h$ are given by

$$
\begin{aligned}
\mathcal{L}_{h Q Q}=-\bar{D} & {\left[\frac{1}{v} M_{D}-\tan \beta\left[\frac{1}{v} M_{D}-\frac{1}{\sqrt{2} s_{\beta}}\left(S_{D}+i P_{D} \gamma_{5}\right)\right] c_{\beta-\alpha}\right] D h } \\
& -\bar{U} \quad\left[\frac{1}{v} M_{U}+\cot \beta\left[\frac{1}{v} M_{U}-\frac{1}{\sqrt{2} c_{\beta}}\left(S_{U}+i P_{U} \gamma_{5}\right)\right] c_{\beta-\alpha}\right] U h
\end{aligned}
$$

where

$$
S_{D} \equiv \frac{1}{2}\left(\eta_{2}^{D}+\eta_{2}^{D \dagger}\right), \quad P_{D} \equiv-\frac{i}{2}\left(\eta_{2}^{D}-\eta_{2}^{D \dagger}\right)
$$

are $3 \times 3$ hermitian matrices and $S_{U}$ and $P_{U}$ are defined similarly by making the replacements $D \rightarrow U$ and $2 \rightarrow 1$. Note that both $h$-mediated FCNC interactions (implicit in the offdiagonal matrix elements of $S$ and $P$ ) and $\mathrm{CP}$-violating interactions proportional to $P$ are suppressed by a factor of $c_{\beta-\alpha}$ in the decoupling limit. Moreover, FCNCs and CP-violating effects mediated by $A$ and $H$ are suppressed by the square of the heavy Higgs masses (relative to $v$ ), due to the propagator suppression. Since $m_{h} \ll m_{H}, m_{A}$ and $c_{\beta-\alpha} \simeq \mathcal{O}\left(v^{2} / m_{A}^{2}\right)$ near the decoupling limit, we see that the flavor-violating processes and CP-violating processes mediated by $h, H$ and $A$ are all suppressed by the same factor. Thus, for $m_{A} \gtrsim \mathcal{O}(1 \mathrm{TeV})$, the decoupling limit provides a viable mechanism for suppressed Higgs-mediated FCNCs and suppressed Higgs-mediated CP-violating effects in the most general 2HDM.

Note that the approach to decoupling can be delayed if either $\tan \beta \gg 1$ or $\cot \beta \gg 1$, as is evident from eq. (49). For example, decoupling at large $\tan \beta$ or $\cot \beta$ occurs when 
$\left|c_{\beta-\alpha} \tan \beta\right| \ll 1$ or $\left|c_{\beta-\alpha} \cot \beta\right| \ll 1$, respectively. Using eqs. (36) and (26), these conditions are respectively equivalent to

$$
m_{A}^{2} \gg\left|\lambda_{6}\right| v^{2} \cot \beta \quad \text { and } \quad m_{A}^{2} \gg\left|\lambda_{7}\right| v^{2} \tan \beta,
$$

which supplement the usual requirement of $m_{A}^{2} \gg \lambda_{i} v^{2}$. That is, there are two possible ranges of the CP-odd Higgs squared-mass, $\lambda_{i} v^{2} \ll m_{A}^{2} \lesssim\left|\lambda_{7}\right| v^{2} \tan \beta$ [or $\lambda_{i} v^{2} \ll m_{A}^{2} \lesssim$ $\left.\left|\lambda_{6}\right| v^{2} \cot \beta\right]$ when $\tan \beta \gg 1$ [or $\cot \beta \gg 1$ ], where the $h$ couplings to $V V$, hh and hhh are nearly indistinguishable from the corresponding $h_{\mathrm{SM}}$ couplings, whereas one of the $h f \bar{f}$ couplings can deviate significantly from the corresponding $h_{\mathrm{SM}} f \bar{f}$ couplings.

The cubic and quartic Higgs self-couplings depend on the parameters of the 2HDM po-

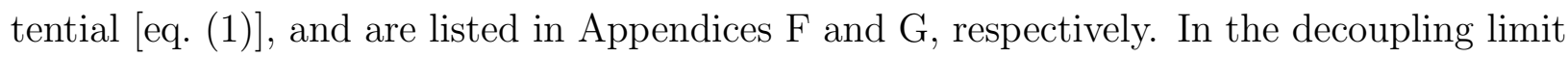
(DL) of $\alpha \rightarrow \beta-\pi / 2$, we denote the terms of the scalar potential corresponding to the cubic Higgs couplings by $\mathcal{V}_{\mathrm{DL}}^{(3)}$ and the terms corresponding to the quartic Higgs couplings by $\mathcal{V}_{\mathrm{DL}}^{(4)}$. The coefficients of the quartic terms in the scalar Higgs potential can be written more simply in terms of the linear combinations of couplings defined earlier [eqs. (25)-(28)] and three additional combinations (see Appendix $\mathrm{E}$ for a discussion of the significance of these combinations):

$$
\begin{aligned}
& \lambda_{T}=\frac{1}{4} s_{2 \beta}^{2}\left(\lambda_{1}+\lambda_{2}\right)+\lambda_{345}\left(s_{\beta}^{4}+c_{\beta}^{4}\right)-2 \lambda_{5}-s_{2 \beta} c_{2 \beta}\left(\lambda_{6}-\lambda_{7}\right), \\
& \lambda_{U}=\frac{1}{2} s_{2 \beta}\left(s_{\beta}^{2} \lambda_{1}-c_{\beta}^{2} \lambda_{2}+c_{2 \beta} \lambda_{345}\right)-\lambda_{6} s_{\beta} s_{3 \beta}-\lambda_{7} c_{\beta} c_{3 \beta} . \\
& \lambda_{V}=\lambda_{1} s_{\beta}^{4}+\lambda_{2} c_{\beta}^{4}+\frac{1}{2} \lambda_{345} s_{2 \beta}^{2}-2 s_{2 \beta}\left(\lambda_{6} s_{\beta}^{2}+\lambda_{7} c_{\beta}^{2}\right) .
\end{aligned}
$$

The resulting expressions for $\mathcal{V}_{\mathrm{DL}}^{(3)}$ and $\mathcal{V}_{\mathrm{DL}}^{(4)}$ are

$$
\begin{aligned}
\mathcal{V}_{\mathrm{DL}}^{(3)}= & \frac{1}{2} \lambda v\left(h^{3}+h G^{2}+2 h G^{+} G^{-}\right)+\left(\lambda_{T}+\lambda_{F}\right) v h H^{+} H^{-} \\
& +\frac{1}{2} \widehat{\lambda} v\left[3 H h^{2}+H G^{2}+2 H G^{+} G^{-}-2 h\left(A G+H^{+} G^{-}+H^{-} G^{+}\right)\right] \\
& +\frac{1}{2} \lambda_{U} v\left(H^{3}+H A^{2}+2 H H^{+} H^{-}\right) \\
& +\left[\lambda_{A}-\lambda+\frac{1}{2} \lambda_{F}\right] v H\left(H^{+} G^{-}+H^{-} G^{+}\right) \\
& +\left(\lambda_{A}-\lambda\right) v H A G+\frac{1}{2} \lambda_{T} v h A^{2}+\left(\lambda-\lambda_{A}+\frac{1}{2} \lambda_{T}\right) v h H^{2} \\
& +\frac{i}{2} \lambda_{F} v A\left(H^{+} G^{-}-H^{-} G^{+}\right),
\end{aligned}
$$


and

$$
\begin{aligned}
\mathcal{V}_{\mathrm{DL}}^{(4)}= & \frac{1}{8} \lambda\left(G^{2}+2 G^{+} G^{-}+h^{2}\right)^{2} \\
+ & \hat{\lambda}\left(h^{3} H-h^{2} A G-h^{2} H^{+} G^{-}-h^{2} H^{-} G^{+}+h H G^{2}+2 h H G^{+} G^{-}-A G^{3}\right. \\
& \left.-2 A G G^{+} G^{-}-G^{2} H^{-} G^{+}-G^{2} H^{+} G^{-}-2 H^{+} G^{-} G^{+} G^{-}-2 H^{-} G^{+} G^{-} G^{+}\right) \\
+ & \frac{1}{2}\left(\lambda_{T}+\lambda_{F}\right)\left(h^{2} H^{+} H^{-}+H^{2} G^{+} G^{-}+A^{2} G^{+} G^{-}+G^{2} H^{+} H^{-}\right) \\
+ & \lambda_{U}\left(h H^{3}+h H A^{2}+2 h H H^{+} H^{-}-H^{2} A G-H^{2} H^{+} G^{-}-H^{2} H^{-} G^{+}-A^{3} G\right. \\
& \left.-A^{2} H^{+} G^{-}-A^{2} H^{-} G^{+}-2 A G H^{+} H^{-}-2 H^{+} H^{-} H^{+} G^{-}-2 H^{-} H^{+} H^{-} G^{+}\right) \\
+ & {\left[2\left(\lambda_{A}-\lambda\right)+\lambda_{F}\right]\left(h H H^{+} G^{-}+h H H^{-} G^{+}-A G H^{+} G^{-}-A G H^{-} G^{+}\right) } \\
+ & \frac{1}{4} \lambda_{V}\left(H^{4}+2 H^{2} A^{2}+A^{4}+4 H^{2} H^{+} H^{-}+4 A^{2} H^{+} H^{-}+4 H^{+} H^{-} H^{+} H^{-}\right) \\
+ & \frac{1}{2}\left(\lambda-\lambda_{A}\right)\left(H^{+} H^{+} G^{-} G^{-}+H^{-} H^{-} G^{+} G^{+}-2 h H A G\right)+\frac{1}{4} \lambda_{T}\left(h^{2} A^{2}+H^{2} G^{2}\right) \\
+ & \frac{1}{4}\left[2\left(\lambda-\lambda_{A}\right)+\lambda_{T}\right]\left(h^{2} H^{2}+A^{2} G^{2}\right)+\left(\lambda-\lambda_{A}+\lambda_{T}\right) H^{+} H^{-} G^{+} G^{-} \\
+ & \frac{i}{2} \lambda_{F}\left(h A H^{+} G^{-}-h A H^{-} G^{+}+H_{G} H^{+} G^{-}-H G H^{-} G^{+}\right),
\end{aligned}
$$

where $G$ and $G^{ \pm}$are the Goldstone bosons (eaten by the $Z$ and $W^{ \pm}$, respectively). Moreover, for $c_{\beta-\alpha}=0$, we have $m_{h}^{2}=\lambda v^{2}$ and $m_{H}^{2}-m_{A}^{2}=\left(\lambda-\lambda_{A}\right) v^{2}$, whereas $m_{H^{ \pm}}^{2}-m_{A}^{2}=\frac{1}{2} \lambda_{F} v^{2}$ is exact at tree-level. As expected, in the decoupling limit, the low-energy effective scalar theory (which includes $h$ and the three Goldstone bosons) is precisely the same as the corresponding SM Higgs theory, with $\lambda$ proportional to the Higgs quartic coupling.

One can use the results of Appendices $\mathrm{F}$ and $\mathrm{G}$ to compute the first non-trivial $\mathcal{O}\left(c_{\beta-\alpha}\right)$ corrections to eqs. (55) and (56) as one moves away from the decoupling limit. These results are given in Tables \and II. For example, the $h h h$ and $h h h h$ couplings in the decoupling limit are given by

$$
\begin{aligned}
& g_{h h h} \simeq-3 v\left(\lambda-3 \widehat{\lambda} c_{\beta-\alpha}\right) \simeq \frac{-3 m_{h}^{2}}{v}+6 \widehat{\lambda} c_{\beta-\alpha} v \\
& g_{h h h h} \simeq-3\left(\lambda-4 \widehat{\lambda} c_{\beta-\alpha}\right) \simeq \frac{-3 m_{h}^{2}}{v^{2}}+9 \widehat{\lambda} c_{\beta-\alpha}
\end{aligned}
$$

where we have used eq. (30). Precision measurements of these couplings could in principle (modulo radiative corrections, which are known within the SM 26]) provide evidence for a departure from the corresponding SM relations. 
Using the explicit forms for the quartic Higgs couplings given in Appendix $G$, it follows that all quartic couplings are $\lesssim \mathcal{O}(1)$ if we require that the $\lambda_{i} \lesssim \mathcal{O}(1)$. Unitarity constraints on Goldstone/Higgs scattering processes can be used to impose numerical limitations on the contributing quartic couplings $19,20,21,22,23]$. If we apply tree-level unitarity constraints for $\sqrt{s}$ larger than all Higgs masses, then $\lambda_{i} / 4 \pi \lesssim \mathcal{O}(1)$ (the precise analytic upper bounds are given in ref. 22]). One can also investigate a less stringent requirement if the Higgs sector is close to the decoupling limit. Namely, assuming $m_{h} \ll m_{H}, m_{A}, m_{H^{ \pm}}$, one can simply impose unitarity constraints on the low-energy effective scalar theory. One must check, for example, that all $2 \rightarrow 2$ scattering processes involving the $W^{ \pm}, Z$ and $h$ satisfy partial-wave unitarity [20, 22, 23]. At tree-level, one simply obtains the well known SM result, $\lambda \leq 8 \pi / 3$, where $\lambda$ is given by eq. (25). ${ }^{12}$ At one-loop, the heavier Higgs scalars can contribute via virtual exchanges, and the restrictions on the self-couplings now involve both the light and the heavier Higgs scalars. For example, in order to avoid large one-loop corrections to the four-point interaction $W^{+} W^{-} \rightarrow h h$ via an intermediate loop of a heavy Higgs pair, the quartic interactions among $h^{2} H^{2}, h^{2} A^{2}$ and $h^{2} H^{+} H^{-}$must be perturbative. In this case, eq. (56) implies that $\left|\lambda-\lambda_{A}\right|,\left|\lambda_{F}\right| \lesssim 1$. It follows that there is a bound on the squared-mass splittings among the heavy Higgs states of $\mathcal{O}\left(v^{2}\right)$. Thus, to maintain unitarity and perturbativity, the decoupling limit demands rather degenerate heavy Higgs bosons.

Using the explicit forms for the cubic Higgs couplings given in Appendix $\mathbf{E}$, it follows that all cubic couplings are $\lesssim \mathcal{O}(v)$ if we require that the $\lambda_{i} \lesssim \mathcal{O}(1)$. The cubic couplings can also be rewritten in terms of the Higgs masses. For example, one possible form for the hhh coupling is given in eq. (F6). Here, we shall consider two equivalent expressions for the $h H^{+} H^{-}$coupling:

$$
\begin{aligned}
& g_{h H^{+} H^{-}}=-\frac{1}{v}\left[\left(m_{h}^{2}-\frac{m_{12}^{2}}{s_{\beta} c_{\beta}}\right) \frac{c_{\beta+\alpha}}{s_{\beta} c_{\beta}}+\left(2 m_{H^{ \pm}}^{2}-m_{h}^{2}\right) s_{\beta-\alpha}+\frac{1}{2} v^{2}\left(\frac{\lambda_{6}}{s_{\beta}^{2}}-\frac{\lambda_{7}}{c_{\beta}^{2}}\right) c_{\beta-\alpha}\right] \\
& =\frac{1}{v}\left[\left(2 m_{A}^{2}-2 m_{H^{ \pm}}^{2}-m_{h}^{2}\right) s_{\beta-\alpha}+2\left(m_{A}^{2}-m_{h}^{2}\right) \frac{c_{2 \beta} c_{\beta-\alpha}}{s_{2 \beta}}+v^{2}\left(\frac{\lambda_{5} c_{\beta+\alpha}}{s_{\beta} c_{\beta}}-\frac{\lambda_{6} s_{\alpha}}{s_{\beta}}+\frac{\lambda_{7} c_{\alpha}}{c_{\beta}}\right)\right] .
\end{aligned}
$$

From the first line of eq. (59)), it appears that $g_{h H^{+} H^{-}}$grows quadratically with the heavy

$\overline{12}$ Using $m_{h}^{2}=\lambda v^{2}$, this bound is a factor of 2 more stringent than that of ref. [20] based on the requirement $\left|\operatorname{Re} a_{0}\right| \leq \frac{1}{2}$ for the $s$-wave partial wave amplitude [27]. 
charged Higgs mass. However, this is an illusion, as can be seen in the subsequent expression for $g_{h H^{+} H^{-}}$. In particular, $m_{A}^{2}-m_{H^{ \pm}}^{2} \sim \mathcal{O}\left(v^{2}\right)$ follows from eq. (11), while in the decoupling limit, $m_{A}^{2} c_{\beta-\alpha} \sim \mathcal{O}\left(v^{2}\right)$ follows from eq. (D3). Hence, $g_{h H^{+} H^{-}} \sim \mathcal{O}(v)$ as expected. One can also check that the apparent singular behavior as $s_{\beta} \rightarrow 0$ or $c_{\beta} \rightarrow 0$ is in fact absent, since the original form of $g_{h H^{+} H^{-}}$was well behaved in this limit. Clearly, the most elegant form for $g_{h H^{+} H^{-}}$is given in eq. (F1). No matter which form is used, it is straightforward to perform an expansion for small $c_{\beta-\alpha}$ to obtain

$$
g_{h H^{+} H^{-}}=-v\left(\lambda_{F}+\lambda_{T}\right)+\mathcal{O}\left(c_{\beta-\alpha}\right)
$$

which agrees with the corresponding result given in Table 1 of Appendix $\mathrm{F}$

One can also be misled by writing the cubic couplings in terms of $\Lambda_{i}$, which are employed in an alternate parameterization of the 2HDM scalar potential given in Appendix A In particular, in the CP-conserving case, $m_{12}^{2}=\frac{1}{2} v^{2} s_{\beta} c_{\beta} \Lambda_{5}$, which becomes large in the approach to the decoupling limit. Consequently, all the $\Lambda_{i}(i=1, \ldots, 6)$ are large in the decoupling limit [see eq. (A3)], even though the magnitudes of the $\lambda_{i}$ are all $\lesssim \mathcal{O}(1)$.

One important consequence of $g_{h H^{+} H^{-}} \sim \mathcal{O}(v)$ is that the one-loop amplitude for $h \rightarrow \gamma \gamma$ reduces to the corresponding SM result in the decoupling limit (where $m_{H^{ \pm}} \gg v$ ). To prove this, we observe that in the decoupling limit, all $h$ couplings to SM particles that enter the one-loop Feynman diagrams for $h \rightarrow \gamma \gamma$ are given by the corresponding SM values. However, there is a new contribution to the one-loop amplitude that arises from a charged Higgs loop. But, this contribution is suppressed by $\mathcal{O}\left(v^{2} / m_{H^{ \pm}}^{2}\right)$ because $g_{h H^{+} H^{-}} \sim \mathcal{O}(v)$, and our assertion is proved. In addition, the first non-trivial corrections to decoupling, of $\mathcal{O}\left(v^{2} / m_{A}^{2}\right)$, can easily be computed and arise from two sources. First, the contribution of the charged Higgs loop yields a contribution to the $h \rightarrow \gamma \gamma$ amplitude proportional to $g_{h H^{+} H^{-}} v / m_{H^{ \pm}}^{2}$. Second, the contribution of the fermion loops are altered due to the modified $h f \bar{f}$ couplings [see eq. (49)], which yield corrections of $\mathcal{O}\left(c_{\beta-\alpha}\right) \sim \mathcal{O}\left(v^{2} / m_{A}^{2}\right)$. Both corrections enter at the same order. Note that the contribution of the $W$ loop is also modified, but the corresponding first order correction is of $\mathcal{O}\left(c_{\beta-\alpha}^{2}\right)$ [since the $h W^{+} W^{-}$coupling is proportional to $s_{\beta-\alpha}$ ] and thus can be neglected.

The above considerations can be generalized to all loop-induced processes which involve the $h$ and SM particles as external states. As long as $\lambda_{i} \lesssim \mathcal{O}(1)$, the Appelquist-Carazzone decoupling theorem [28] guarantees that for $m_{A} \rightarrow \infty$, the amplitudes for such processes 
approach the corresponding SM values. The same result also applies to radiatively-corrected $h$ decay rates and cross-sections.

\section{A SM-LIKE HIGGS BOSON WITHOUT DECOUPLING}

We have demonstrated above that the decoupling limit (where $m_{A}^{2} \gg\left|\lambda_{i}\right| v^{2}$ ) implies that $\left|c_{\beta-\alpha}\right| \ll 1$. However, the $\left|c_{\beta-\alpha}\right| \ll 1$ limit is more general than the decoupling limit. From eq. (36), one learns that $\left|c_{\beta-\alpha}\right| \ll 1$ implies that either (i) $m_{A}^{2} \gg \lambda_{A} v^{2}$, and/or (ii) $|\widehat{\lambda}| \ll 1$ subject to the condition specified by eq. (33). Case (i) is the decoupling limit described in Section 3. Although case (ii) is compatible with $m_{A}^{2} \gg \lambda_{i} v^{2}$, which is the true decoupling limit, there is no requirement a priori that $m_{A}$ be particularly large [as long as eq. (33) is satisfied]. It is even possible to have $m_{A}<m_{h}$, implying that all Higgs boson masses are $\lesssim \mathcal{O}(v)$, in contrast to the true decoupling limit. In this latter case, there does not exist an effective low-energy scalar theory consisting of a single Higgs boson.

Although the tree-level couplings of $h$ to vector bosons may appear to be SM-like, a significant deviation of either the $h \bar{D} D$ or $h \bar{U} U$ coupling from the corresponding SM value is possible. For example, for $\left|c_{\beta-\alpha}\right| \ll 1$, the $h$ couplings to quark pairs normalized to their SM values [see eqs. (36), (45) and (46)] are given by:

$$
h \bar{D} D: \quad 1-\frac{\widehat{\lambda} v^{2} \tan \beta}{m_{A}^{2}-\lambda_{A} v^{2}}, \quad h \bar{U} U: \quad 1+\frac{\widehat{\lambda} v^{2} \cot \beta}{m_{A}^{2}-\lambda_{A} v^{2}} .
$$

If $m_{A} \lesssim \mathcal{O}(v)$ and $\tan \beta \gg 1[\cot \beta \gg 1]$, then the deviation of the $h \bar{D} D[h \bar{U} U]$ coupling from the corresponding SM value can be significant even though $|\widehat{\lambda}| \ll 1$. A particularly nasty case is one where the $h \bar{D} D[h \bar{U} U]$ coupling is equal in magnitude but opposite in sign to the corresponding SM value 29]. ${ }^{13}$ For example, the $h \bar{D} D$ coupling of eq. (61) is equal to -1 when $\tan \beta \simeq 2\left[\left(m_{A}^{2} / v^{2}\right)-\lambda_{A}\right] / \widehat{\lambda} \gg 1$. Of course, the latter corresponds to an isolated point of the parameter space; it is far more likely that the $h \bar{D} D$ coupling will exhibit a discernible deviation in magnitude from its SM value.

Even if the tree-level couplings of $h$ to both vector bosons and fermions appear to be SM-like, radiative corrections can introduce deviations from SM expectations [29] if $m_{A}$ is

${ }^{13}$ Note that for $|\widehat{\lambda}| \ll 1$ [i.e., for $\left|c_{\beta-\alpha}\right| \ll 1$ with $m_{A}$ arbitrary, where the $h V V$ couplings are SM-like], there is no choice of parameters for which both the $h \bar{D} D$ and $h \bar{U} U$ couplings are equal in magnitude but opposite in sign relative to the corresponding SM couplings. 
not significantly larger than $v{ }^{14}$ For example, consider the amplitude for $h \rightarrow \gamma \gamma$ (which corresponds to a dimension-five effective operator). If $m_{A} \lesssim \mathcal{O}(v)$ [implying that $m_{H^{ \pm}} \sim$ $\mathcal{O}(v)$ ] and $|\widehat{\lambda}| \ll 1$ (implying that tree-level couplings of $h$ approach their SM values), then the charged Higgs boson loop contribution to the $h \rightarrow \gamma \gamma$ amplitude will not be suppressed. Hence the resulting amplitude will be shifted from the SM result, thus revealing that true decoupling has not been achieved, and the $h$ is not the SM Higgs boson [29].

Radiative corrections can also introduce deviations from SM expectations if the Higgs self-coupling parameters are large [30]. We can illustrate this in a model in which $h$ is SMlike and all other Higgs bosons are very heavy, and yet the decoupling limit does not apply. Consider a model in which $m_{12}^{2}=\lambda_{6}=\lambda_{7}=0$ and the Higgs potential parameters are chosen to yield $m_{H}=m_{A}=m_{H^{ \pm}}$and $c_{\beta-\alpha}=0$. This can be achieved by taking $m_{11}^{2}=m_{22}^{2}$ $\operatorname{and}^{15}$

$$
\lambda_{1}=\lambda_{3}+\frac{\lambda_{5} c_{2 \beta}}{c_{\beta}^{2}} \quad \lambda_{2}=\lambda_{3}-\frac{\lambda_{5} c_{2 \beta}}{s_{\beta}^{2}} \quad \lambda_{4}=\lambda_{5}
$$

with $\lambda_{5}<0$ and $-\left(\lambda_{1} \lambda_{2}\right)^{1 / 2}<\lambda_{345}<0$ [thereby ensuring that $m_{A}^{2}>0, m_{h}<m_{H}$ and eq. (41) are satisfied]. These results are most easily obtained by using eqs. (D20)-(D23). One immediately finds that $m_{h}^{2}=\left(\lambda_{3}+\lambda_{5}\right) v^{2}$ and $m_{H}^{2}=m_{A}^{2}=m_{H^{ \pm}}^{2}=-\lambda_{5} v^{2}$. It is easy to check that $\hat{\lambda}=0$ is exact, which yields $c_{\beta-\alpha}=0$ (since $\lambda_{345}<0$ implies that $m_{A}^{2}>m_{L}^{2}$ and $m_{h}^{2}=m_{L}^{2}\left[c f\right.$. eqs. (19) and (20)]), and $\lambda=\lambda_{A}=\lambda_{3}+\lambda_{5}$. Note that although $\hat{\lambda}=c_{\beta-\alpha}=0$, eq. (36) implies that the ratio $\hat{\lambda} / c_{\beta-\alpha}=-\lambda_{345}=\left(m_{A}^{2}-m_{h}^{2}\right) / v^{2}$ can be taken to be an arbitrary positive parameter. This example exhibits a model in which the properties of $h$ are indistinguishable from those of the SM Higgs boson, but the decoupling limit can never be achieved ( since $m_{12}^{2}=0$ ). One cannot take the masses of the mass-degenerate $H, A$ and $H^{ \pm}$arbitrarily large with $m_{h} \sim \mathcal{O}\left(m_{Z}\right)$ without taking all the $\left|\lambda_{i}\right|(i=1, \ldots, 5)$ arbitrarily large (thereby violating unitarity). Nevertheless, if one takes the $\left|\lambda_{i}\right|$ close to their unitarity limits, one can find a region of parameter space in which $m_{H}=m_{A}=m_{H^{ \pm}} \gg m_{h} \sim \mathcal{O}\left(m_{Z}\right)$. If only $h$ were observed, it would appear to be difficult to distinguish this case from a Higgs sector close to the decoupling limit. However, when

\footnotetext{
${ }^{14}$ Radiative corrections that contribute to shifts in the coefficients of operators of dimension $\leq 4$ will simply renormalize the parameters of the scalar potential. Hence the deviation from the SM of the properties of $h$ associated with dimension $\leq 4$ operators will continue to be suppressed in the limit of the renormalized parameter $|\widehat{\lambda}| \ll 1$.

${ }^{15}$ In this case, eqs. (6) and (7) imply that $\tan ^{2} \beta=\left(\lambda_{345}-\lambda_{1}\right) /\left(\lambda_{345}-\lambda_{2}\right)$.
} 
the $\left|\lambda_{i}\right|$ are large one expects large radiative corrections due to loops that depend on the Higgs self-couplings. For example, the one-loop corrections to the hhh coupling (which at tree-level is given by $g_{h h h}=-3 m_{h}^{2} / v^{2}$ when $\left.c_{\beta-\alpha}=0\right)$ can deviate by as much as $100 \%$ or more from the corresponding corrections in the Standard Model in the above model where $c_{\beta-\alpha}=0$ and $m_{H}=m_{A}=m_{H^{ \pm}} \gg m_{h} \sim \mathcal{O}\left(m_{Z}\right)$ [30]. More generally, a model with a light SM-like Higgs boson and all other Higgs bosons heavy could be distinguished from a Higgs sector near the decoupling limit only by observing the effects of one-loop corrections proportional to the (large) Higgs self-coupling parameters. Such radiative corrections could deviate significantly from the corresponding loop corrections in the Standard Model.

Two additional examples in which the $|\widehat{\lambda}| \ll 1$ limit is realized are given by:

1. $\tan \beta \gg 1, \lambda_{6}=\lambda_{7}=0$, and $m_{A}^{2}>\left(\lambda_{2}-\lambda_{5}\right) v^{2}$, and

2. $\lambda_{1}=\lambda_{2}=\lambda_{345}, \lambda_{6}=\lambda_{7}=0$, and $m_{A}^{2}>\left(\lambda_{2}-\lambda_{5}\right) v^{2}[31]$.

The condition on $m_{A}^{2}$ in the two cases is required by eq. (333). In case $1, \hat{\lambda}=0$ when $\beta=\pi / 2$, whereas in case $2, \hat{\lambda}=0$ independently of $\tan \beta$. In both these cases, it is straightforward to use eqs. (12) and (16) to obtain

$$
m_{h, H}^{2}=\left\{\begin{array}{l}
\lambda_{2} v^{2} \\
m_{A}^{2}+\lambda_{5} v^{2}
\end{array}\right.
$$

Since $m_{L}^{2}=\lambda_{2} v^{2}$, eq. (20) yields $\cos (\beta-\alpha)=0$ as expected.

Two special limits of case 2 above are treated in ref. [31], where scalar potentials with $\lambda_{1}=\lambda_{2}=\lambda_{3}=\mp \lambda_{4}= \pm \lambda_{5}>0$ (and $\lambda_{6}=\lambda_{7}=0$ ) are considered. Assuming that $m_{A}^{2}>\left(\lambda_{2}-\lambda_{5}\right) v^{2}$, the resulting Higgs spectrum is given by $m_{H^{ \pm}}^{2}=m_{H}^{2}=m_{A}^{2} \pm m_{h}^{2}$ and $m_{h}^{2}=\lambda_{1} v^{2}\left(m_{A}^{2}\right.$ is a free parameter that depends on $\left.m_{12}^{2}\right)$. In the case of $\lambda_{5}>0$, one has $m_{A}^{2}>0$ and it is possible to have a Higgs spectrum in which $A$ is very light, while the other Higgs bosons (including $h$ ) are heavy and approximately degenerate in mass. In the case of $\lambda_{5}<0$, one has $m_{A}^{2}>2 m_{h}^{2}$, and a light $A$ would imply that all the Higgs bosons of the model are light. In both cases $c_{\beta-\alpha}=0$, and the tree-level couplings of $h$ correspond precisely to those of the SM Higgs boson [see Section IV]. These are clearly very special cases, corresponding to a distinctive form of the quartic terms of the Higgs potential:

$$
\mathcal{V}_{4}=\frac{1}{2} \lambda_{1}\left[\left(\Phi_{1}^{\dagger} \Phi_{1}+\Phi_{2}^{\dagger} \Phi_{2}\right)^{2} \pm\left(\Phi_{1}^{\dagger} \Phi_{2}-\Phi_{2}^{\dagger} \Phi_{1}\right)^{2}\right]
$$


where the choice of sign corresponds to the sign of $\lambda_{5}$. Note that $\mathcal{V}_{4}$ above exhibits a flat direction if $\lambda_{5}>0$, whereas the scalar potential possesses a globally stable minimum if $\lambda_{5}<0$ [see eq. (4)]

Next, we examine a region of Higgs parameter space where $|\sin (\beta-\alpha)| \ll 1$, in which the heavier CP-even Higgs boson, $H$ is SM-like (also considered in refs. [29] and [31]). In this case, the $h$ couplings to vector boson pairs are highly suppressed. This is far from the decoupling regime. Nevertheless, this region does merit a closer examination, which we now perform.

When $s_{\beta-\alpha} \rightarrow 0$, we have $\beta-\alpha=0$ or $\pi$. We shall work to first nontrivial order in a $s_{\beta-\alpha}$ expansion, with $c_{\beta-\alpha} \simeq \pm\left(1-\frac{1}{2} s_{\beta-\alpha}^{2}\right)$. Using the results of eqs. (D9)-(D11) and eq. (11), we obtain: ${ }^{16}$

$$
\begin{aligned}
m_{A}^{2} & \simeq v^{2}\left[\mp \frac{\widehat{\lambda}}{s_{\beta-\alpha}}+\lambda_{A} \pm \frac{3}{2} \widehat{\lambda} s_{\beta-\alpha}\right] \\
m_{h}^{2} & \simeq v^{2}\left[\mp \frac{\widehat{\lambda}}{s_{\beta-\alpha}}+\lambda \pm \frac{1}{2} \widehat{\lambda} s_{\beta-\alpha}\right] \simeq m_{A}^{2}+\left(\lambda-\lambda_{A} \mp \hat{\lambda} s_{\beta-\alpha}\right) v^{2} \\
m_{H}^{2} & \simeq v^{2}\left(\lambda \pm \widehat{\lambda} s_{\beta-\alpha}\right) \\
m_{H^{ \pm}}^{2} & \simeq v^{2}\left[\mp \frac{\widehat{\lambda}}{s_{\beta-\alpha}}+\lambda_{A}+\frac{1}{2} \lambda_{F} \pm \frac{3}{2} \widehat{\lambda} s_{\beta-\alpha}\right]=m_{A}^{2}+\frac{1}{2} \lambda_{F} v^{2}
\end{aligned}
$$

The condition $m_{H}>m_{h}$ imposes the inequality (valid to first order in $s_{\beta-\alpha}$ ):

$$
m_{A}^{2}<v^{2}\left(\lambda_{A} \pm 2 \widehat{\lambda} s_{\beta-\alpha}\right)
$$

[cf. eq. (D32)]. Note that eq. (69) implies that all Higgs squared-masses are of $\mathcal{O}\left(v^{2}\right)$. We may also use eq. (10) to obtain

$$
m_{12}^{2} \simeq v^{2} s_{\beta} c_{\beta}\left[\mp \frac{\hat{\lambda}}{s_{\beta-\alpha}}+\lambda_{A}+\lambda_{5}+\frac{1}{2} \lambda_{6} t_{\beta}^{-1}+\frac{1}{2} \lambda_{7} t_{\beta} \pm \frac{3}{2} \widehat{\lambda} s_{\beta-\alpha}\right] .
$$

We can rewrite eq. (65) in another form [or equivalently use eqs. (D30) and (D31) to obtain]:

$$
\sin (\beta-\alpha) \simeq \frac{\mp \widehat{\lambda} v^{2}}{m_{A}^{2}-\lambda_{A} v^{2}} \simeq \frac{ \pm \hat{\lambda} v^{2}}{m_{H}^{2}-m_{h}^{2}}
$$

\footnotetext{
${ }^{16}$ Note that eqs. (D4) and (D5) are interchanged under the transformation $m_{h}^{2} \leftrightarrow m_{H}^{2}$ and $c_{\beta-\alpha} \leftrightarrow-s_{\beta-\alpha}$. Thus, applying these transformations to eqs. (29)-(32) yields the results given in eqs. (65)-(68) with $c_{\beta-\alpha}=+1$.
} 
The $\left|s_{\beta-\alpha}\right| \ll 1$ limit is achieved when $|\widehat{\lambda}| \ll 1$, subject to the condition given in eq. (69). Clearly, $H$ is SM-like, since if $s_{\beta-\alpha} \simeq 0$, then the couplings of $H$ to $V V, H H$ and $H H H$ coincide with the corresponding SM Higgs boson couplings. ${ }^{17}$

The couplings of $H$ to fermion pairs are obtained from eq. (44) by expanding the Yukawa couplings of $H$ to $\mathcal{O}\left(s_{\beta-\alpha}\right)$

$$
\begin{aligned}
\mathcal{L}_{H Q Q}= & -\bar{D}\left[ \pm \frac{1}{v} M_{D}+\tan \beta\left[\frac{1}{v} M_{D}-\frac{1}{\sqrt{2} s_{\beta}}\left(S_{D}+i P_{D} \gamma_{5}\right)\right] s_{\beta-\alpha}\right] D H \\
& -\bar{U}\left[ \pm \frac{1}{v} M_{U}-\cot \beta\left[\frac{1}{v} M_{U}-\frac{1}{\sqrt{2} c_{\beta}}\left(S_{U}+i P_{U} \gamma_{5}\right)\right] s_{\beta-\alpha}\right] U H
\end{aligned}
$$

where \pm corresponds to $c_{\beta-\alpha}= \pm 1$ and $S_{D}$ and $P_{D}$ are given by eq. (1501). If $|\widehat{\lambda}| \tan \beta \ll 1$ or $|\widehat{\lambda}| \cot \beta \ll 1$, then the $H f \bar{f}$ couplings reduce to the corresponding $h_{\mathrm{SM}} f \bar{f}$ couplings. However, if $|\widehat{\lambda}| \ll 1 \lesssim|\widehat{\lambda}| \tan \beta[$ or $|\widehat{\lambda}| \ll 1 \lesssim|\widehat{\lambda}| \cot \beta]$ when $\tan \beta \gg 1$ [or $\cot \beta \gg 1$ ], then the $H f \bar{f}$ couplings can deviate significantly from the corresponding $h_{\mathrm{SM}} f \bar{f}$ couplings. This behavior is qualitatively different from the decoupling limit, where for fixed $\lambda_{i}$ and large $\tan \beta$ [or large $\cot \beta$ ], one can always choose $m_{A}$ large enough such that the $h f \bar{f}$ couplings approach the corresponding SM values. In contrast, when $\left|s_{\beta-\alpha}\right| \ll 1$, the size of $m_{A}$ is restricted by eq. (69), and so there is no guarantee of SM-like $\operatorname{Hf} \bar{f}$ couplings when either $\tan \beta$ or $\cot \beta$ is large.

Although the tree-level properties of $H$ are SM-like when $|\widehat{\lambda}| \ll 1$, deviations can occur for loop-induced processes as noted earlier. Again, the $H \rightarrow \gamma \gamma$ amplitude will deviate from the corresponding SM amplitude due to the contribution of the charged Higgs loop which is not suppressed since $m_{H^{ \pm}} \sim \mathcal{O}(v)$. Thus, departures from true decoupling can in principle be detected for $\left|s_{\beta-\alpha}\right| \ll 1$.

We now briefly examine some model examples in which $\left|s_{\beta-\alpha}\right| \ll 1$ is realized. These examples are closely related to the ones previously considered in the case of $c_{\beta-\alpha}=0$. First, consider the model in which $m_{12}^{2}=\lambda_{6}=\lambda_{7}=0$ and the Higgs potential parameters are chosen to yield $m_{h}=m_{A}=m_{H^{ \pm}}$and $s_{\beta-\alpha}=0$. This can be achieved by taking $m_{11}^{2}=m_{22}^{2}$ and the non-zero $\lambda_{i}$ given by eq. (62) with $\lambda_{5}<0$ and $\lambda_{345}>0$. In this case, $m_{H}^{2}=\left(\lambda_{3}+\lambda_{5}\right) v^{2}$ and $m_{h}^{2}=m_{A}^{2}=m_{H^{ \pm}}^{2}=-\lambda_{5} v^{2}$. It is easy to check that $\hat{\lambda}=0$ is exact and yields $s_{\beta-\alpha}=0$

${ }^{17}$ When $\hat{\lambda}=0$, the $H$ couplings to $V V, H H$, and $f \bar{f}$ [see eq. (72)] all differ by an overall sign from the corresponding $h_{\mathrm{SM}}$ couplings if $c_{\beta-\alpha}=-1$. However, this sign is unphysical, since one can eliminate it with a redefinition $h \rightarrow-h$ and $H \rightarrow-H$, which is equivalent to replacing $\alpha$ with $\alpha \pm \pi$. 
(since $\lambda_{345}>0$ implies that $m_{A}^{2}<m_{L}^{2}$ and $m_{H}^{2}=m_{L}^{2}[c f$. eqs. (19) and (20)]). Thus, the properties of $H$ are indistinguishable from those of the SM Higgs boson. However, all the other mass-degenerate Higgs bosons are lighter than the SM-like Higgs boson, $H$. Thus, one expects that all Higgs bosons can be observed (once the SM-like Higgs boson is discovered). That is, there is little chance of confusing $H$ with the Higgs boson of the Standard Model.

Two additional examples in which the $\left|s_{\beta-\alpha}\right| \ll 1$ limit is realized are given by:

1. $\tan \beta \gg 1, \lambda_{6}=\lambda_{7}=0$, and $m_{A}^{2}<\left(\lambda_{2}-\lambda_{5}\right) v^{2}$, and

2. $\lambda_{1}=\lambda_{2}=\lambda_{345}, \lambda_{6}=\lambda_{7}=0$, and $m_{A}^{2}<\left(\lambda_{2}-\lambda_{5}\right) v^{2}[31]$.

The condition on $m_{A}^{2}$ in the two cases is required by eq. (69). In case $1, \hat{\lambda}=0$ when $\beta=\pi / 2$, whereas in case $2, \hat{\lambda}=0$ independently of $\tan \beta$. In both these cases, it is straightforward to use eqs. (12) and (16) to obtain

$$
m_{h, H}^{2}=\left\{\begin{array}{l}
m_{A}^{2}+\lambda_{5} v^{2} \\
\lambda_{2} v^{2}
\end{array}\right.
$$

Since $m_{L}^{2}=\lambda_{2} v^{2}$, it follows from eq. (20) that $c_{\beta-\alpha}^{2}=1$. Hence, $\sin (\beta-\alpha)=0$, which implies that $H$ is SM-like. ${ }^{18}$

Finally, we note that the SM-like Higgs bosons resulting from the limiting cases above where $\hat{\lambda}=0$ can be easily understood in terms of the squared-mass matrix entries of eqs. (12) and (13). In order to achieve $c_{\beta-\alpha}=0$ or $s_{\beta-\alpha}=0$, we demand that $\tan 2 \beta=\tan 2 \alpha$. This implies [see eq. (17)] that the entries in the $\mathcal{B}^{2}$ matrix be in the same ratio as the entries in the term proportional to $m_{A}^{2}$ in eq. (12):

$$
\frac{2 \mathcal{M}_{12}^{2}}{\mathcal{M}_{11}^{2}-\mathcal{M}_{22}^{2}}=\tan 2 \beta
$$

It is easy to check that

$$
\widehat{\lambda} v^{2}=\frac{1}{2}\left(\mathcal{B}_{11}^{2}-\mathcal{B}_{22}^{2}\right) \sin 2 \beta-\mathcal{B}_{12}^{2} \cos 2 \beta
$$

Eqs. (12) and (74) immediately imply that $\hat{\lambda}=0$ is equivalent to $\tan 2 \beta=\tan 2 \alpha$. Moreover, to determine whether $c_{\beta-\alpha}=0$ or $s_{\beta-\alpha}=0$, simply note that if the $\operatorname{sign}$ of $\sin 2 \alpha / \sin 2 \beta$ is

$\overline{18 \text { Since }} \lambda_{6}=\lambda_{7}=0$, if we additionally set $m_{12}^{2}=0$, then we recover the discrete symmetry of the Higgs potential previously noted in Section $\amalg$ Thus, there is no true decoupling limit in this model. Moreover, since $m_{A}^{2}=-\lambda_{5} v^{2}$ (which implies that $\lambda_{5}<0$ ), eq. (73) yields $m_{h}=0$, although this result would be modified once radiative corrections are included. 
negative [positive], then $c_{\beta-\alpha}=0\left[s_{\beta-\alpha}=0\right]$. In the convention where $\tan \beta$ is positive, it follows that $\sin 2 \beta>0$. Using eqs. (12) and (13), if the sign of

$$
\mathcal{M}_{12}^{2}=s_{\beta} c_{\beta}\left[\left(\lambda_{345}-\lambda_{5}\right) v^{2}-m_{A}^{2}\right]+v^{2}\left(\lambda_{6} c_{\beta}^{2}+\lambda_{7} s_{\beta}^{2}\right)
$$

is negative [positive], then $c_{\beta-\alpha}=0\left[s_{\beta-\alpha}=0\right]$. One can check that the conditions given by eqs. (33) and (69) correspond precisely to the negative [positive] sign of $\mathcal{M}_{12}^{2}$ [eq. (76)], after

imposing $\widehat{\lambda}=0 .{ }^{19}$ The condition $\hat{\lambda}=0$ can be achieved not only for appropriate choices of the $\lambda_{i}$ and $\tan \beta$ in the general $2 \mathrm{HDM}$, but also can be fulfilled in the MSSM when radiative corrections are incorporated (see Section 6).

\section{DECOUPLING EFFECTS IN THE MSSM HIGGS SECTOR}

The Higgs sector of the MSSM is a CP-conserving two-Higgs-doublet model, with a Higgs potential whose dimension-four terms respect supersymmetry and with type-II Higgsfermion couplings. The quartic couplings $\lambda_{i}$ are given by $[9]$

$$
\lambda_{1}=\lambda_{2}=-\lambda_{345}=\frac{1}{4}\left(g^{2}+g^{\prime 2}\right), \quad \lambda_{4}=-\frac{1}{2} g^{2}, \quad \lambda_{5}=\lambda_{6}=\lambda_{7}=0 .
$$

The squared-mass parameters defined in eq. (18) simplify to: $m_{L}^{2}=m_{Z}^{2} \cos ^{2} 2 \beta, m_{D}^{2}=0$, $m_{T}^{2}=m_{Z}^{2}$ and $m_{S}^{2}=m_{A}^{2}+m_{Z}^{2}$. Using eq. (77), the invariant coupling parameters defined in eqs. (25)-(28) and eqs. (52)-(54) reduce to

$$
\begin{aligned}
\lambda=-\lambda_{T}=\lambda_{V} & =\frac{1}{4}\left(g^{2}+g^{2}\right) \cos ^{2} 2 \beta, \\
\hat{\lambda}=-\lambda_{U} & =\frac{1}{4}\left(g^{2}+g^{2}\right) \sin 2 \beta \cos 2 \beta, \\
\lambda_{A} & =\frac{1}{4}\left(g^{2}+g^{\prime 2}\right) \cos 4 \beta, \\
\lambda_{F} & =\frac{1}{2} g^{2} .
\end{aligned}
$$

The results of Section 2 can then be used to obtain the well known tree-level results:

$$
m_{A}^{2}=m_{12}^{2}(\tan \beta+\cot \beta), \quad m_{H^{ \pm}}^{2}=m_{A}^{2}+m_{W}^{2},
$$

and a neutral CP-even squared-mass matrix given by

$$
\mathcal{M}_{0}^{2}=\left(\begin{array}{ll}
m_{A}^{2} \sin ^{2} \beta+m_{Z}^{2} \cos ^{2} \beta & -\left(m_{A}^{2}+m_{Z}^{2}\right) \sin \beta \cos \beta \\
-\left(m_{A}^{2}+m_{Z}^{2}\right) \sin \beta \cos \beta & m_{A}^{2} \cos ^{2} \beta+m_{Z}^{2} \sin ^{2} \beta
\end{array}\right)
$$

${ }^{19}$ It is simplest to use $\widehat{\lambda}=0$ to eliminate the quantity $\lambda_{1} c_{\beta}^{2}-\lambda_{2} s_{\beta}^{2}$ from $\lambda_{A}$ in eqs. (33) and (69). 
with eigenvalues

$$
m_{H^{0}, h^{0}}^{2}=\frac{1}{2}\left(m_{A}^{2}+m_{Z}^{2} \pm \sqrt{\left(m_{A}^{2}+m_{Z}^{2}\right)^{2}-4 m_{Z}^{2} m_{A}^{2} \cos ^{2} 2 \beta}\right)
$$

and the diagonalizing angle $\alpha$ given by

$$
\cos 2 \alpha=-\cos 2 \beta\left(\frac{m_{A}^{2}-m_{Z}^{2}}{m_{H^{0}}^{2}-m_{h^{0}}^{2}}\right), \quad \sin 2 \alpha=-\sin 2 \beta\left(\frac{m_{H^{0}}^{2}+m_{h^{0}}^{2}}{m_{H^{0}}^{2}-m_{h^{0}}^{2}}\right) .
$$

One can also write

$$
\cos ^{2}(\beta-\alpha)=\frac{m_{h}^{2}\left(m_{Z}^{2}-m_{h}^{2}\right)}{m_{A}^{2}\left(m_{H}^{2}-m_{h}^{2}\right)} .
$$

In the decoupling limit where $m_{A} \gg m_{Z}$, the above formulae yield

$$
\begin{aligned}
m_{h}^{2} \simeq m_{Z}^{2} \cos ^{2} 2 \beta, & m_{H}^{2} \simeq m_{A}^{2}+m_{Z}^{2} \sin ^{2} 2 \beta \\
m_{H^{ \pm}}^{2}=m_{A}^{2}+m_{W}^{2}, & \cos ^{2}(\beta-\alpha) \simeq \frac{m_{Z}^{4} \sin ^{2} 4 \beta}{4 m_{A}^{4}}
\end{aligned}
$$

That is, $m_{A} \simeq m_{H} \simeq m_{H^{ \pm}}$, up to corrections of $\mathcal{O}\left(m_{Z}^{2} / m_{A}\right)$, and $\cos (\beta-\alpha)=0$ up to corrections of $\mathcal{O}\left(m_{Z}^{2} / m_{A}^{2}\right)$.

It is straightforward to work out all the tree-level Higgs couplings, both in general and in the decoupling limit. Since the Higgs-fermion couplings follow the type-II pattern, the Higgs-fermion Yukawa couplings are given by eq. (44) with $\eta_{1}^{U}=\eta_{2}^{D}=0$. However, oneloop radiative corrections can lead in some cases to significant shifts from the tree-level couplings. It is of interest to examine how the approach to the decoupling limit is affected by the inclusion of radiative corrections.

First, we note that in some cases, one-loop effects mediated by loops of supersymmetric particles can generate a deviation from Standard Model expectations, even if $m_{A} \gg m_{Z}$ where the corrections to the decoupling limit are negligible. As a simple example, if squarks are relatively light, then squark loop contributions to the $h \rightarrow g g$ and $h \rightarrow \gamma \gamma$ amplitudes can be significant [32]. Of course, in the limit of large squark masses, the contributions of the supersymmetric loops decouple as well [33]. Thus, in the MSSM, there are two separate decoupling limits that must be analyzed. For simplicity, we assume henceforth that supersymmetric particle masses are large (say of order $1 \mathrm{TeV}$ ), so that supersymmetric loop effects of the type just mentioned are negligible.

The leading contributions to the radiatively-corrected Higgs couplings arise in two ways. First, the radiative corrections to the CP-even Higgs squared-mass matrix results in a shift 
of the CP-even Higgs mixing angle $\alpha$ from its tree-level value. That is, the dominant Higgs propagator corrections can to a good approximation be absorbed into an effective ("radiatively-corrected") mixing angle $\alpha$ [34]. In this approximation, we can write:

$$
\mathcal{M}^{2} \equiv\left(\begin{array}{cc}
\mathcal{M}_{11}^{2} & \mathcal{M}_{12}^{2} \\
\mathcal{M}_{12}^{2} & \mathcal{M}_{22}^{2}
\end{array}\right)=\mathcal{M}_{0}^{2}+\delta \mathcal{M}^{2},
$$

where the tree-level contribution $\mathcal{M}_{0}^{2}$ was given in eq. (800) and $\delta \mathcal{M}^{2}$ is the contribution from the radiative corrections. Then, $\cos (\beta-\alpha)$ is given by

$$
\begin{aligned}
\cos (\beta-\alpha) & =\frac{\left(\mathcal{M}_{11}^{2}-\mathcal{M}_{22}^{2}\right) \sin 2 \beta-2 \mathcal{M}_{12}^{2} \cos 2 \beta}{2\left(m_{H}^{2}-m_{h}^{2}\right) \sin (\beta-\alpha)} \\
& =\frac{m_{Z}^{2} \sin 4 \beta+\left(\delta \mathcal{M}_{11}^{2}-\delta \mathcal{M}_{22}^{2}\right) \sin 2 \beta-2 \delta \mathcal{M}_{12}^{2} \cos 2 \beta}{2\left(m_{H}^{2}-m_{h}^{2}\right) \sin (\beta-\alpha)}
\end{aligned}
$$

Using tree-level Higgs couplings with $\alpha$ replaced by its renormalized value provides a useful first approximation to the radiatively-corrected Higgs couplings.

Second, contributions from the one-loop vertex corrections to tree-level Higgs-fermion couplings can modify these couplings in a significant way, especially in the limit of large $\tan \beta$. In particular, although the tree-level Higgs-fermion coupling follow the type-II pattern, when radiative corrections are included, all possible dimension-four Higgs-fermion couplings are generated. These results can be summarized by an effective Lagrangian that describes the coupling of the neutral Higgs bosons to the third generation quarks:

$$
-\mathcal{L}_{\text {eff }}=\left[\left(h_{b}+\delta h_{b}\right) \bar{b}_{R} b_{L} \Phi_{1}^{0 *}+\left(h_{t}+\delta h_{t}\right) \bar{t}_{R} t_{L} \Phi_{2}^{0}\right]+\Delta h_{t} \bar{t}_{R} t_{L} \Phi_{1}^{0}+\Delta h_{b} \bar{b}_{R} b_{L} \Phi_{2}^{0 *}+\text { h.c. }
$$

resulting in a modification of the tree-level relation between $h_{t}\left[h_{b}\right]$ and $m_{t}\left[m_{b}\right]$ as follows $[35$, 36, 37, 38]:

$$
\begin{aligned}
& m_{b}=\frac{h_{b} v}{\sqrt{2}} \cos \beta\left(1+\frac{\delta h_{b}}{h_{b}}+\frac{\Delta h_{b} \tan \beta}{h_{b}}\right) \equiv \frac{h_{b} v}{\sqrt{2}} \cos \beta\left(1+\Delta_{b}\right), \\
& m_{t}=\frac{h_{t} v}{\sqrt{2}} \sin \beta\left(1+\frac{\delta h_{t}}{h_{t}}+\frac{\Delta h_{t} \cot \beta}{h_{t}}\right) \equiv \frac{h_{t} v}{\sqrt{2}} \sin \beta\left(1+\Delta_{t}\right) .
\end{aligned}
$$

The dominant contributions to $\Delta_{b}$ are $\tan \beta$-enhanced, with $\Delta_{b} \simeq\left(\Delta h_{b} / h_{b}\right) \tan \beta$; for $\tan \beta \gg 1, \delta h_{b} / h_{b}$ provides a small correction to $\Delta_{b}$. [In the same limit, $\Delta_{t} \simeq \delta h_{t} / h_{t}$, with the additional contribution of $\left(\Delta h_{t} / h_{t}\right) \cot \beta$ providing a small correction.] 
From eq. (87) we can obtain the couplings of the physical neutral Higgs bosons to third generation quarks. The resulting interaction Lagrangian is of the form:

$$
\mathcal{L}_{\text {int }}=-\sum_{q=t, b}\left[g_{h q \bar{q}} h q \bar{q}+g_{H q \bar{q}} H q \bar{q}-i g_{A q \bar{q}} A \bar{q} \gamma_{5} q\right] .
$$

Using eqs. (88) and (89), one obtains [39, 40]:

$$
\begin{aligned}
g_{h b \bar{b}} & =-\frac{m_{b}}{v} \frac{\sin \alpha}{\cos \beta}\left[1+\frac{1}{1+\Delta_{b}}\left(\frac{\delta h_{b}}{h_{b}}-\Delta_{b}\right)(1+\cot \alpha \cot \beta)\right], \\
g_{H b \bar{b}} & =\frac{m_{b}}{v} \frac{\cos \alpha}{\cos \beta}\left[1+\frac{1}{1+\Delta_{b}}\left(\frac{\delta h_{b}}{h_{b}}-\Delta_{b}\right)(1-\tan \alpha \cot \beta)\right] \\
g_{A b \bar{b}} & =\frac{m_{b}}{v} \tan \beta\left[1+\frac{1}{\left(1+\Delta_{b}\right) \sin ^{2} \beta}\left(\frac{\delta h_{b}}{h_{b}}-\Delta_{b}\right)\right] \\
g_{h t \bar{t}} & =\frac{m_{t}}{v} \frac{\cos \alpha}{\sin \beta}\left[1-\frac{1}{1+\Delta_{t}} \frac{\Delta h_{t}}{h_{t}}(\cot \beta+\tan \alpha)\right] \\
g_{H t \bar{t}} & =\frac{m_{t}}{v} \frac{\sin \alpha}{\sin \beta}\left[1-\frac{1}{1+\Delta_{t}} \frac{\Delta h_{t}}{h_{t}}(\cot \beta-\cot \alpha)\right] \\
g_{A t \bar{t}} & =\frac{m_{t}}{v} \cot \beta\left[1-\frac{1}{1+\Delta_{t}} \frac{\Delta h_{t}}{h_{t}}(\cot \beta+\tan \beta)\right] .
\end{aligned}
$$

We now turn to the decoupling limit. First consider the implications for the radiativelycorrected value of $\cos (\beta-\alpha)$. Since $\delta \mathcal{M}_{i j}^{2} \sim \mathcal{O}\left(m_{Z}^{2}\right)$, and $m_{H}^{2}-m_{h}^{2}=m_{A}^{2}+\mathcal{O}\left(m_{Z}^{2}\right)$, one finds [39]

$$
\cos (\beta-\alpha)=c\left[\frac{m_{Z}^{2} \sin 4 \beta}{2 m_{A}^{2}}+\mathcal{O}\left(\frac{m_{Z}^{4}}{m_{A}^{4}}\right)\right],
$$

in the limit of $m_{A} \gg m_{Z}$, where

$$
c \equiv 1+\frac{\delta \mathcal{M}_{11}^{2}-\delta \mathcal{M}_{22}^{2}}{2 m_{Z}^{2} \cos 2 \beta}-\frac{\delta \mathcal{M}_{12}^{2}}{m_{Z}^{2} \sin 2 \beta} .
$$

The effect of the radiative corrections has been to modify the tree-level definition of $\widehat{\lambda}$ :

$$
\widehat{\lambda} v^{2}=c m_{Z}^{2} \sin 2 \beta \cos 2 \beta .
$$

Eq. (97) exhibits the expected decoupling behavior for $m_{A} \gg m_{Z}$. However, eqs. (866) and (97) exhibit another way in which $\cos (\beta-\alpha)=0$ can be achieved - simply choose the MSSM parameters (that govern the Higgs mass radiative corrections) such that the numerator of eq. (86) vanishes. That is,

$$
2 m_{Z}^{2} \sin 2 \beta=2 \delta \mathcal{M}_{12}^{2}-\tan 2 \beta\left(\delta \mathcal{M}_{11}^{2}-\delta \mathcal{M}_{22}^{2}\right)
$$


This condition is equivalent to $c=0$, and thus corresponds precisely to the case of $\widehat{\lambda}=0$ discussed at the beginning of Section 5. Although $\hat{\lambda} \neq 0$ at tree-level, the above analysis shows that $|\widehat{\lambda}| \ll 1$ can arise due to the effects of one-loop radiative corrections that approximately cancel the tree-level result. ${ }^{20}$ In particular, eq. (100) is independent of the value of $m_{A}$. Typically, eq. (100) yields a solution at large $\tan \beta$. That is, by approximating $\tan 2 \beta \simeq-\sin 2 \beta \simeq-2 / \tan \beta$, one can determine the value of $\beta$ at which $\hat{\lambda} \simeq 0[39]$ :

$$
\tan \beta \simeq \frac{2 m_{Z}^{2}-\delta \mathcal{M}_{11}^{2}+\delta \mathcal{M}_{22}^{2}}{\delta \mathcal{M}_{12}^{2}} .
$$

Hence, there exists a value of $\tan \beta$ (which depends on the choice of MSSM parameters) where $\cos (\beta-\alpha) \simeq 0$ independently of the value of $m_{A}$. If $m_{A}$ is not much larger than $m_{Z}$, then $h$ is a SM-like Higgs boson outside the decoupling regime. ${ }^{21}$ Of course, as explained in Section 5, this SM-like Higgs boson can be distinguished in principle from the SM Higgs boson by measuring its decay rate to two photons and looking for a deviation from SM predictions.

Finally, we analyze the radiatively-corrected Higgs-fermion couplings [eqs. (91)-(96)] in the decoupling limit. Here it is useful to note that for $m_{A} \gg m_{Z}$,

$$
\cot \alpha=-\tan \beta-\frac{2 m_{Z}^{2}}{m_{A}^{2}} \tan \beta \cos 2 \beta+\mathcal{O}\left(\frac{m_{Z}^{4}}{m_{A}^{4}}\right) .
$$

Applying this result to eqs. (91) and (94), it follows that in the decoupling limit, $g_{h q \bar{q}}=$ $g_{h_{\mathrm{SM}} q \bar{q}}=m_{q} / v$. Away from the decoupling limit, the Higgs couplings to down-type fermions can deviate significantly from their tree-level values due to enhanced radiative corrections at large $\tan \beta\left[\right.$ where $\left.\Delta_{b} \simeq \mathcal{O}(1)\right]$. In particular, because $\Delta_{b} \propto \tan \beta$, the leading one-loop radiative correction to $g_{h b \bar{b}}$ is of $\mathcal{O}\left(m_{Z}^{2} \tan \beta / m_{A}^{2}\right)$, which formally decouples only when $m_{A}^{2} \gg$ $m_{Z}^{2} \tan \beta$. This behavior is called delayed decoupling in ref. [41], although this phenomenon can also occur in a more general 2HDM (with tree-level couplings), as noted previously in Section 4 [below eq. (150)].

${ }^{20}$ The one-loop corrections arise from the exchange of supersymmetric particles, whose contributions can be enhanced for certain MSSM parameter choices. One can show that the two-loop corrections are subdominant, so that the approximation scheme is under control.

${ }^{21}$ For large $\tan \beta$ and $m_{A} \lesssim \mathcal{O}\left(m_{Z}\right)$, one finds that $\sin (\beta-\alpha) \simeq 0$, implying that $H$ is the SM-like Higgs boson, as discussed in Section 5. 


\section{DISCUSSION AND CONCLUSIONS}

In this paper, we have studied the decoupling limit of a general CP-conserving two-Higgsdoublet model. In this limit, the lightest Higgs boson of the model is a CP-even neutral Higgs scalar $(h)$ with couplings identical to those of the SM Higgs boson. Near the decoupling limit, the first order corrections for the Higgs couplings to gauge and Higgs bosons, the Higgs-fermion Yukawa couplings and the Higgs cubic and quartic self-couplings have also been obtained. These results exhibit a definite pattern for the deviations of the $h$ couplings from those of the SM Higgs boson. In particular, the rate of the approach to decoupling depends on the particular Higgs coupling as follows:

$$
\begin{aligned}
\frac{g_{h V V}^{2}}{g_{h_{\mathrm{SM}} V V}^{2}} & \simeq 1-\frac{\widehat{\lambda}^{2} v^{4}}{m_{A}^{4}}, \\
\frac{g_{h h h}^{2}}{g_{h_{\mathrm{SM}}}^{2} h_{\mathrm{SM}} h_{\mathrm{SM}}} & \simeq 1-\frac{6 \widehat{\lambda}^{2} v^{2}}{\lambda m_{A}^{2}}, \\
\frac{g_{h t t}^{2}}{g_{h_{\mathrm{SM}} t t}^{2}} & \simeq 1+\frac{2 \widehat{\lambda} v^{2} \cot \beta}{m_{A}^{2}}\left(1-\xi_{t}\right), \\
\frac{g_{h b b}^{2}}{g_{h_{\mathrm{SM}}}^{2}} & \simeq 1-\frac{2 \widehat{\lambda} v^{2} \tan \beta}{m_{A}^{2}}\left(1-\xi_{b}\right),
\end{aligned}
$$

where $\xi_{t}$ and $\xi_{b}$ reflect the terms proportional to $S$ and $P$ in eq. (49). Thus, the approach to decoupling is fastest for the $h$ couplings to vector bosons and slowest for the couplings to down-type (or up-type) quarks if $\tan \beta>1$ (or $\tan \beta<1$ ). We may apply the above results to the MSSM (see Section 6). Including the leading $\tan \beta$-enhanced radiative corrections, $\xi_{b}=v \Delta h_{b} /\left(\sqrt{2} s_{\beta} m_{b}\right)=\Delta_{b} /\left(s_{\beta}^{2}\left(1+\Delta_{b}\right)\right)$ [whereas $\xi_{t} \ll 1$ can be neglected] and $\hat{\lambda}$ is given by eq. (99). Plugging into eqs. (103)-(106), one reproduces the results obtained in ref. 39].

Although the results of this paper were derived from a tree-level analysis of couplings, these results can also be applied to the radiatively-corrected couplings that multiply operators of dimension-four or less. An example of this was given in Section 6, where we showed how the decoupling limit applies to the radiatively-corrected Higgs-fermion Yukawa couplings. In particular, near the decoupling limit one can neglect radiative corrections that are generated by the exchange of heavy Higgs bosons. These contributions are suppressed by a loop factor in addition to the suppression factor of $\mathcal{O}\left(v^{2} / m_{A}^{2}\right)$ and thus are smaller than corrections to tree-level Higgs couplings that enter at first order in $c_{\beta-\alpha}$. This should be contrasted with loop-induced Higgs couplings (e.g., $h \rightarrow \gamma \gamma$ which is generated by a 
dimension-five effective operator), where the corrections of $\mathcal{O}\left(c_{\beta-\alpha}\right)$ to tree-level Higgs couplings that appear in the one-loop amplitude and the effects of a heavy Higgs boson loop are both of $\mathcal{O}\left(v^{2} / m_{A}^{2}\right)$ [in addition to the overall one-loop factor]. Consequently, both contributions are equally important in determining the overall correction to the loop-induced Higgs couplings due to the departure from the decoupling limit.

If a neutral Higgs boson, $h$, is discovered at a future collider, it may turn out that its couplings are close to those expected of the SM Higgs boson. The challenge for future experiments is then to determine whether the observed state is the SM Higgs boson, or whether it is the lowest lying scalar state of a non-minimal Higgs sector [42]. If the latter, then it is likely that the additional scalar states of the model are heavy, and the decoupling limit applies. In this case, it is possible that the heavier scalars cannot be detected at the LHC or at an $e^{+} e^{-}$linear collider (LC) with a center-of-mass energy in the range of 350$800 \mathrm{GeV}$. Moreover, it may not be possible to distinguish between the $h$ and the SM-Higgs boson at the LHC. However, the measurements of Higgs observables at the LC can provide sufficient precision to observe deviations from SM Higgs properties at the few percent level. In this case, one can begin to probe deep into the decoupling regime [12].

In this paper, we also clarified a Higgs parameter regime in which $h$ possesses SM-like couplings to vector bosons but where $m_{A}^{2} \lesssim \mathcal{O}\left(v^{2}\right)$ and the decoupling limit does not apply (see Section 5). In this case, the couplings of $h$ to fermion pairs can deviate significantly from the corresponding SM Higgs-fermion couplings if either $\tan \beta$ or $\cot \beta$ is large. Moreover, the masses of $H, A$ and $H^{ \pm}$are not particularly large, and all scalars would be accessible at the LHC and/or the LC.

The discovery of the Higgs boson will be a remarkable achievement. Nevertheless, the lesson of the decoupling limit is that a SM-like Higgs boson provides very little information about the nature of the underlying electroweak symmetry-breaking dynamics. It is essential to find evidence for departures from SM Higgs predictions. Such departures can reveal crucial information about the existence of a non-minimal Higgs sector. Precision Higgs measurements can also provide critical tests of possible new physics beyond the Standard Model. As an example, in the MSSM, deviations in Higgs couplings from the decoupling limit can yield indirect information about the MSSM parameters. In particular, at large tan $\beta$ the sensitivity to MSSM parameters may be increased due to enhanced radiative corrections. The decoupling limit is both a curse and an opportunity. If nature chooses the Higgs sector 
parameters to lie deep in the decoupling regime, then it may not be possible to distinguish the observed $h$ from the SM Higgs boson. On the other hand, given sufficient precision of the measurements of $h$ branching ratios and cross-sections [40], it may be possible to observe a small but statistically significant deviation from SM expectations, and provide a first glimpse of the physics responsible for electroweak symmetry breaking.

\section{Acknowledgments}

We are grateful to Scott Thomas and Chung Kao for their contributions at the initial stages of this project. We also appreciate a number of useful conversations with Maria Krawczyk. Finally, we would like to thank Marcela Carena, Heather Logan and Steve Mrenna for various insights concerning the supersymmetric applications based on the results of this paper. This work was supported in part by the U.S. Department of Energy.

\section{APPENDIX A: AN ALTERNATIVE PARAMETERIZATION OF THE 2HDM} SCALAR POTENTIAL

In this Appendix, we give the translation of the parameters of eq. (II) employed in this paper to the parameters employed in the Higgs Hunter's Guide (HHG) [7]. While the HHG parameterization was useful for some purposes (e.g., the scalar potential minimum is explicitly exhibited), it obscures the decoupling limit.

In the HHG parameterization, the most general 2HDM scalar potential, subject to a discrete symmetry $\Phi_{1} \rightarrow-\Phi_{1}$ that is only softly violated by dimension-two terms, is given by $^{22}$

$$
\begin{aligned}
\mathcal{V} & =\Lambda_{1}\left(\Phi_{1}^{\dagger} \Phi_{1}-V_{1}^{2}\right)^{2}+\Lambda_{2}\left(\Phi_{2}^{\dagger} \Phi_{2}-V_{2}^{2}\right)^{2}+\Lambda_{3}\left[\left(\Phi_{1}^{\dagger} \Phi_{1}-V_{1}^{2}\right)+\left(\Phi_{2}^{\dagger} \Phi_{2}-V_{2}^{2}\right)\right]^{2} \\
& +\Lambda_{4}\left[\left(\Phi_{1}^{\dagger} \Phi_{1}\right)\left(\Phi_{2}^{\dagger} \Phi_{2}\right)-\left(\Phi_{1}^{\dagger} \Phi_{2}\right)\left(\Phi_{2}^{\dagger} \Phi_{1}\right)\right]+\Lambda_{5}\left[\operatorname{Re}\left(\Phi_{1}^{\dagger} \Phi_{2}\right)-\mathrm{V}_{1} \mathrm{~V}_{2} \cos \xi\right]^{2} \\
& +\Lambda_{6}\left[\operatorname{Im}\left(\Phi_{1}^{\dagger} \Phi_{2}\right)-\mathrm{V}_{1} \mathrm{~V}_{2} \sin \xi\right]^{2} \\
& +\Lambda_{7}\left[\operatorname{Re}\left(\Phi_{1}^{\dagger} \Phi_{2}\right)-\mathrm{V}_{1} \mathrm{~V}_{2} \cos \xi\right]\left[\operatorname{Im}\left(\Phi_{1}^{\dagger} \Phi_{2}\right)-\mathrm{V}_{1} \mathrm{~V}_{2} \sin \xi\right]
\end{aligned}
$$

22 In the HHG, the $V_{i}$ and $\Lambda_{i}$ are denoted by $v_{i}$ and $\lambda_{i}$, respectively. In eq. (A1), we employ the former notation in order to distinguish between the HHG parameterization and the notation of eqs. (11) and (5). 
where the $\Lambda_{i}$ are real parameters. ${ }^{23}$ The $V_{1,2}$ are related to the $v_{1,2}$ of eq. (15) by $V_{1,2}=$ $v_{1,2} / \sqrt{2}$. The conversion from these $\Lambda_{i}$ to the $\lambda_{i}$ and $m_{i j}^{2}$ of eq. (11) is:

$$
\begin{aligned}
& \lambda_{1}=2\left(\Lambda_{1}+\Lambda_{3}\right), \\
& \lambda_{2}=2\left(\Lambda_{2}+\Lambda_{3}\right), \\
& \lambda_{3}=2 \Lambda_{3}+\Lambda_{4}, \\
& \lambda_{4}=-\Lambda_{4}+\frac{1}{2}\left(\Lambda_{5}+\Lambda_{6}\right), \\
& \lambda_{5}=\frac{1}{2}\left(\Lambda_{5}-\Lambda_{6}-i \Lambda_{7}\right), \\
& \lambda_{6}=\lambda_{7}=0 \\
& m_{11}^{2}=-2 V_{1}^{2} \Lambda_{1}-2\left(V_{1}^{2}+V_{2}^{2}\right) \Lambda_{3}, \\
& m_{22}^{2}=-2 V_{2}^{2} \Lambda_{2}-2\left(V_{1}^{2}+V_{2}^{2}\right) \Lambda_{3}, \\
& m_{12}^{2}=V_{1} V_{2}\left(\Lambda_{5} \cos \xi-i \Lambda_{6} \sin \xi-\frac{i}{2} e^{i \xi} \Lambda_{7}\right) .
\end{aligned}
$$

Excluding $\lambda_{6}$ and $\lambda_{7}$, the scalar potential [eqs. (11) and [A1)] are fixed by ten real parameters. The CP-conserving limit of eq. (A1) is most easily obtained by setting $\xi=0$ and $\Lambda_{7}=0$. In the CP-conserving limit, it is easy to invert eq. (A2) and solve for the $\Lambda_{i}(i=1, \ldots, 6)$. The result is:

$$
\begin{aligned}
& \Lambda_{1}=\frac{1}{2}\left[\lambda_{1}-\lambda_{345}+2 m_{12}^{2} /\left(v^{2} s_{\beta} c_{\beta}\right)\right], \\
& \Lambda_{2}=\frac{1}{2}\left[\lambda_{2}-\lambda_{345}+2 m_{12}^{2} /\left(v^{2} s_{\beta} c_{\beta}\right)\right], \\
& \Lambda_{3}=\frac{1}{2}\left[\lambda_{345}-2 m_{12}^{2} /\left(v^{2} s_{\beta} c_{\beta}\right)\right] \\
& \Lambda_{4}=2 m_{12}^{2} /\left(v^{2} s_{\beta} c_{\beta}\right)-\lambda_{4}-\lambda_{5}, \\
& \Lambda_{5}=2 m_{12}^{2} /\left(v^{2} s_{\beta} c_{\beta}\right) \\
& \Lambda_{6}=2 m_{12}^{2} /\left(v^{2} s_{\beta} c_{\beta}\right)-2 \lambda_{5}
\end{aligned}
$$

where $\lambda_{345} \equiv \lambda_{3}+\lambda_{4}+\lambda_{5}$ and $v^{2} s_{\beta} c_{\beta}=2 V_{1} V_{2}$.

In eq. (A1) we include the $\Lambda_{7}$ term that was left out in the hardcover edition of the HHG. See the erratum that has been included in the paperback edition of the HHG (Perseus Publishing, Cambridge, MA, 2000). 


\section{APPENDIX B: CONDITIONS FOR CP CONSERVATION IN THE TWO-HIGGS DOUBLET MODEL}

First, we derive the conditions such that the Higgs sector does not exhibit explicit CP violation. ${ }^{24}$ It is convenient to adopt a convention in which one of the vacuum expectation values, say $v_{1}$ is real and positive. ${ }^{25}$ This still leaves one additional phase redefinition for the Higgs doublet fields. If there is no explicit CP violation, it should be possible to choose the phases of the Higgs fields so that there are no explicit phases in the Higgs potential parameters of eq. (11). If we consider $\Phi_{1}^{\dagger} \Phi_{2} \rightarrow e^{-i \eta} \Phi_{1}^{\dagger} \Phi_{2}$, then the $\eta$-dependent terms in $\mathcal{V}$ are given by

$$
\begin{aligned}
& \mathcal{V} \ni-m_{12}^{2} e^{-i \eta} \Phi_{1}^{\dagger} \Phi_{2}+\frac{1}{2} \lambda_{5} e^{-2 i \eta}\left(\Phi_{1}^{\dagger} \Phi_{2}\right)^{2} \\
& +\lambda_{6} e^{-i \eta}\left(\Phi_{1}^{\dagger} \Phi_{1}\right)\left(\Phi_{1}^{\dagger} \Phi_{2}\right)+\lambda_{7} e^{-i \eta}\left(\Phi_{2}^{\dagger} \Phi_{2}\right)\left(\Phi_{1}^{\dagger} \Phi_{2}\right)+\text { h.c. }
\end{aligned}
$$

Let us write

$$
m_{12}^{2}=\left|m_{12}^{2}\right| e^{i \theta_{m}}, \quad \lambda_{5,6,7}=\left|\lambda_{5,6,7}\right| e^{i \theta_{5,6,7}} .
$$

Then, all explicit parameter phases are removed if

$$
\theta_{m}-\eta=n_{m} \pi, \quad \theta_{5}-2 \eta=n_{5} \pi, \quad \theta_{6,7}-\eta=n_{6,7} \pi,
$$

where $n_{m, 5,6,7}$ are integers. Writing $\eta=\theta_{m}-n_{m} \pi$ from the first condition of eq. (B3), and substituting into the other conditions, gives

$$
\begin{aligned}
\theta_{5}-2 \theta_{m}=\left(n_{5}-2 n_{m}\right) \pi & \Rightarrow \operatorname{Im}\left[\left(m_{12}^{2}\right)^{2} \lambda_{5}^{*}\right]=0 \\
\theta_{6}-\theta_{m}=\left(n_{6}-n_{m}\right) \pi & \Rightarrow \operatorname{Im}\left[m_{12}^{2} \lambda_{6}^{*}\right]=0 \\
\theta_{7}-\theta_{m}=\left(n_{7}-n_{m}\right) \pi & \Rightarrow \operatorname{Im}\left[m_{12}^{2} \lambda_{7}^{*}\right]=0
\end{aligned}
$$

Eqs. (B4) $-(\underline{B} 6)$ constitute the conditions for the absence of explicit $\mathrm{CP}$ violation in the (tree-level) Higgs sector. A useful convention is one in which $m_{12}^{2}$ is real (by a suitable choice of the phase $\eta$ ). It then follows that $\lambda_{5}, \lambda_{6}$ and $\lambda_{7}$ are also real. Henceforth, we shall assume that all parameters in the scalar potential are real.

\footnotetext{
${ }^{24}$ For another approach, in which invariants are employed to identify basis-independent conditions for CP violation in the Higgs sector, see refs. [43] and [44].

${ }^{25}$ Due to the U(1)-hypercharge symmetry of the theory, it is always possible to make a phase rotation on the scalar fields such that $v_{1}>0$.
} 
Let us consider now the conditions for the absence of spontaneous CP violation. ${ }^{26}$ Let us write $\left\langle\Phi_{1}^{\dagger} \Phi_{2}\right\rangle=\frac{1}{2} v_{1} v_{2} e^{i \xi}$ with $v_{1}$ and $v_{2}$ real and positive and $0 \leq \xi \leq \pi$. The $\xi$-dependent terms in $\mathcal{V}$ are given by

$$
\mathcal{V} \ni-m_{12}^{2} v_{1} v_{2} \cos \xi+\frac{1}{4} \lambda_{5} v_{1}^{2} v_{2}^{2} \cos 2 \xi+\frac{1}{2} \lambda_{6} v_{1}^{3} v_{2} \cos \xi+\frac{1}{2} \lambda_{7} v_{2}^{3} v_{1} \cos \xi
$$

which yields

$$
\frac{\partial \mathcal{V}}{\partial \cos \xi}=-m_{12}^{2} v_{1} v_{2}+\lambda_{5} v_{1}^{2} v_{2}^{2} \cos \xi+\frac{1}{2} \lambda_{6} v_{1}^{3} v_{2}+\frac{1}{2} \lambda_{7} v_{2}^{3} v_{1}
$$

and

$$
\frac{\partial^{2} \mathcal{V}}{\partial(\cos \xi)^{2}}=\lambda_{5} v_{1}^{2} v_{2}^{2} .
$$

Spontaneous CP violation occurs when $\xi \neq 0, \pi / 2$ or $\pi$ at the potential minimum. That is, $\lambda_{5}>0$ and there exists a CP-violating solution to

$$
\cos \xi=\frac{m_{12}^{2}-\frac{1}{2} \lambda_{6} v_{1}^{2}-\frac{1}{2} \lambda_{7} v_{2}^{2}}{\lambda_{5} v_{1} v_{2}}
$$

Thus, we conclude that the criterion for spontaneous CP violation (in a convention where all parameters of the scalar potential are real) is

$$
0 \neq\left|m_{12}^{2}-\frac{1}{2} \lambda_{6} v_{1}^{2}-\frac{1}{2} \lambda_{7} v_{2}^{2}\right|<\lambda_{5} v_{1} v_{2} \quad \text { and } \lambda_{5}>0
$$

Otherwise, the minimum of the potential occurs either at $\xi=0, \pi / 2$ or $\pi$ and $\mathrm{CP}$ is conserved. ${ }^{27}$ The case of $\xi=\pi / 2$ is singular and arises when $m_{12}^{2}=\frac{1}{2} \lambda_{6} v_{1}^{2}+\frac{1}{2} \lambda_{7} v_{2}^{2}$ and $\lambda_{5}>0 .^{28}$ It is convenient to choose a convention where $\left\langle\Phi_{1}^{0}\right\rangle$ is real and $\left\langle\Phi_{2}^{0}\right\rangle$ is pure imaginary. One must then re-evaluate the Higgs mass eigenstates. As shown in ref. [47], the neutral Goldstone boson is now a linear combination of $\operatorname{Im} \Phi_{1}^{0}$ and $\operatorname{Re} \Phi_{2}^{0}$, while the physical CP-odd scalar, $A$ corresponds to the orthogonal combination. The two CP-even Higgs scalars are orthogonal linear combinations of $\operatorname{Re} \Phi_{1}^{0}$ and $\operatorname{Im} \Phi_{2}^{0}$. Most of the results of

26 Similar considerations can be found in refs. 44, 45, 46] and [13].

27 The CP-conserving minimum corresponding to $\xi=0$ or $\xi=\pi$ does not in general correspond to an extremum in $V(\cos \xi)$. Specifically, for $\lambda_{5}<0$, the extremum corresponds to a maximum in $\mathcal{V}$, while for $\lambda_{5}>0$, the extremum corresponding to a minimum of $\mathcal{V}(\cos \xi)$ arises for $|\cos \xi|>1$. In both cases, when restricted to the physical region corresponding to $|\cos \xi| \leq 1$, the minimum of $\mathcal{V}(\cos \xi)$ is attained on the boundary, $|\cos \xi|=1$.

28 Note that the case of $\xi=\pi / 2$ arises automatically in the case of the discrete symmetry discussed in Section III $m_{12}^{2}=\lambda_{6}=\lambda_{7}=0$, when $\lambda_{5}>0$. 
this paper do not apply for this case without substantial revision. Nevertheless, it is clear that the decoupling limit $\left(m_{A}^{2} \gg \lambda_{i} v^{2}\right)$ does not exist due to the condition on $m_{12}^{2}$.

We shall not consider the $\xi=\pi / 2$ model further in this paper. Then, if the parameters of the scalar potential are real and if there is no spontaneous CP-violation, then it is always possible to choose the phase $\eta$ in eq. (B1) so that the potential minimum corresponds to $\xi=0 .{ }^{29}$ In this convention,

$$
\begin{array}{ll}
m_{12}^{2}-\frac{1}{2} \lambda_{6} v_{1}^{2}-\frac{1}{2} \lambda_{7} v_{2}^{2} \geq \lambda_{5} v_{1} v_{2} & \text { for } \lambda_{5}>0, \\
m_{12}^{2}-\frac{1}{2} \lambda_{6} v_{1}^{2}-\frac{1}{2} \lambda_{7} v_{2}^{2} \geq 0 & \text { for } \lambda_{5} \leq 0,
\end{array}
$$

where eq. (B12) follows from eq. (B11), and eq. (B13) is a consequence of the requirement that $\mathcal{V}(\xi=0) \leq \mathcal{V}(\xi=\pi)$. Since $\xi=0$ and both $v_{1}$ and $v_{2}$ are real and positive, this convention corresponds to the one chosen below eq. (91). Note that if we rewrite eq. (10) as 30

$$
m_{A}^{2}=\frac{v^{2}}{v_{1} v_{2}}\left[m_{12}^{2}-\lambda_{5} v_{1} v_{2}-\frac{1}{2} \lambda_{6} v_{1}^{2}-\frac{1}{2} \lambda_{7} v_{2}^{2}\right]
$$

it follows that if $\lambda_{5}>0$, then the condition $m_{A}^{2} \geq 0$ is equivalent to eq. (B12). However, if $\lambda_{5} \leq 0$, then eq. (B13) implies that $m_{A}^{2} \geq\left|\lambda_{5}\right| v^{2}$.

\section{APPENDIX C: A SINGULAR LIMIT: $m_{h}=m_{H}$}

By definition, $m_{h} \leq m_{H}$. The limiting case of $m_{h}=m_{H}$ is special and requires careful treatment in some cases. For example, despite the appearance of $m_{H}^{2}-m_{h}^{2}$ in the denominator of eq. (20), one can show that $0 \leq c_{\beta-\alpha}^{2} \leq 1$. To prove this, we first write

$$
c_{\beta-\alpha}^{2}=\frac{1}{2}\left[1-\frac{m_{S}^{2}-2 m_{L}^{2}}{\sqrt{m_{S}^{4}-4 m_{A}^{2} m_{L}^{2}-4 m_{D}^{4}}}\right] .
$$

Next, we use eq. (18) to explicitly compute:

$$
m_{S}^{4}-4 m_{A}^{2} m_{L}^{2}-4 m_{D}^{4}=m_{A}^{4}-2 m_{A}^{2}\left[\left(\mathcal{B}_{22}^{2}-\mathcal{B}_{11}^{2}\right) c_{2 \beta}+2 \mathcal{B}_{12}^{2} s_{2 \beta}\right]+\left(\mathcal{B}_{11}^{2}-\mathcal{B}_{22}^{2}\right)^{2}+4\left[\mathcal{B}_{12}^{2}\right]^{2}
$$

${ }^{29}$ In particular, if $\xi=\pi$, simply choose $\eta=\pi$, which corresponds to changing the overall sign of $\Phi_{1}^{\dagger} \Phi_{2}$. This is equivalent to redefining the parameters $m_{12}^{2} \rightarrow-m_{12}^{2}, \lambda_{6} \rightarrow-\lambda_{6}$ and $\lambda_{7} \rightarrow-\lambda_{7}$.

${ }^{30}$ Under the assumption that $v_{1}$ and $v_{2}$ are positive, eq. (10) implicitly employs the convention in which $\xi=0$. 
and

$$
\left(m_{S}^{2}-2 m_{L}^{2}\right)^{2}=m_{S}^{4}-4 m_{A}^{2} m_{L}^{2}-4 m_{D}^{4}-\left[\left(\mathcal{B}_{11}^{2}-\mathcal{B}_{22}^{2}\right) s_{2 \beta}-2 \mathcal{B}_{12}^{2} c_{2 \beta}\right]^{2} .
$$

Note that eq. (C2), viewed as a quadratic function of $m_{A}^{2}$ (of the form $A m_{A}^{4}+B m_{A}^{2}+C$ ), is non-negative if $B^{2}-4 A C=\left[\left(\mathcal{B}_{11}^{2}-\mathcal{B}_{22}^{2}\right) s_{2 \beta}-2 \mathcal{B}_{12}^{2} c_{2 \beta}\right]^{2} \geq 0$. It then follows from eq. (C1) that $0 \leq c_{\beta-\alpha}^{2} \leq 1$ if

$$
\left(m_{S}^{2}-2 m_{L}^{2}\right)^{2} \leq m_{S}^{4}-4 m_{A}^{2} m_{L}^{2}-4 m_{D}^{4}
$$

a result which is manifestly true [see eq. (C3)].

We now turn to the case of $m_{h}=m_{H}$. This can arise if and only if the CP-even Higgs squared-mass matrix (in any basis) is proportional to the unit matrix. From eq. (12), it then follows that:

$$
\mathcal{B}_{11}^{2}-\mathcal{B}_{22}^{2}=m_{A}^{2} c_{2 \beta}, \quad 2 \mathcal{B}_{12}^{2}=m_{A}^{2} s_{2 \beta}
$$

where $m_{h}^{2}=m_{H}^{2}=\mathcal{B}_{11}^{2}+m_{A}^{2} s_{\beta}^{2}=\mathcal{B}_{22}^{2}+m_{A}^{2} c_{\beta}^{2}$. Alternatively, from eq. (19), the condition for $m_{h}=m_{H}$ is given by $m_{S}^{4}-4 m_{A}^{2} m_{L}^{2}-4 m_{D}^{4} \equiv A m_{A}^{4}+B m_{A}^{2}+C=0$. However, one must check that this quadratic equation possesses a positive (real) solution for $m_{A}^{2}$. Noting the discussion above eq. (C4), such a solution can exist if and only if $B^{2}-4 A C=0$, which is indeed consistent with eq. (C5). Of course, the results of eq. (C5) are not compatible with the decoupling limit, since it is not possible to have $m_{h}=m_{H}$ and $m_{A}^{2} \gg\left|\lambda_{i}\right| v^{2}$.

If we take $B^{2}-4 A C=0$ but keep $m_{A}$ arbitrary, then eq. (C1) yields

$$
c_{\beta-\alpha}^{2}= \begin{cases}0, & \text { if } m_{L}^{2}<\frac{1}{2} m_{S}^{2}, \\ 1, & \text { if } m_{L}^{2}>\frac{1}{2} m_{S}^{2} .\end{cases}
$$

For $m_{L}^{2}=\frac{1}{2} m_{S}^{2}$, we have $m_{h}^{2}=m_{H}^{2}=\frac{1}{2} m_{S}^{2}$, and the angle $\alpha$ is not well-defined. In this case, one cannot distinguish between $h$ and $H$ in either production or decays, and the corresponding squared-amplitudes should be (incoherently) added in all processes. It is easy to check that the undetermined angle $\alpha$ that appears in the relevant Higgs couplings would then drop out in any such sum of squared-amplitudes. The singular point of parameter space corresponding to $m_{h}=m_{H}$ will not be considered further in this paper. 


\section{APPENDIX D: RELATIONS AMONG HIGGS POTENTIAL PARAMETERS AND MASSES}

It is useful to express the physical Higgs masses in terms of the parameters of the scalar potential [eq. (11)]. First, inserting eqs. (12) and (13) into eq. (15) and examining the diagonal elements yields the CP-even Higgs boson squared-masses:

$$
\begin{gathered}
m_{h}^{2}=m_{A}^{2} c_{\beta-\alpha}^{2}+v^{2}\left[\lambda_{1} c_{\beta}^{2} s_{\alpha}^{2}+\lambda_{2} s_{\beta}^{2} c_{\alpha}^{2}-2 \lambda_{345} c_{\alpha} c_{\beta} s_{\alpha} s_{\beta}+\lambda_{5} c_{\beta-\alpha}^{2}\right. \\
\left.-2 \lambda_{6} c_{\beta} s_{\alpha} c_{\beta+\alpha}+2 \lambda_{7} s_{\beta} c_{\alpha} c_{\beta+\alpha}\right] \\
m_{H}^{2}=m_{A}^{2} s_{\beta-\alpha}^{2}+v^{2}\left[\lambda_{1} c_{\beta}^{2} c_{\alpha}^{2}+\lambda_{2} s_{\beta}^{2} s_{\alpha}^{2}+2 \lambda_{345} c_{\alpha} c_{\beta} s_{\alpha} s_{\beta}+\lambda_{5} s_{\beta-\alpha}^{2}\right. \\
\left.+2 \lambda_{6} c_{\beta} c_{\alpha} s_{\beta+\alpha}+2 \lambda_{7} s_{\beta} s_{\alpha} s_{\beta+\alpha}\right],
\end{gathered}
$$

while the requirement that the off-diagonal entries in eq. (15) are zero yields

$$
\begin{gathered}
m_{A}^{2} s_{\beta-\alpha} c_{\beta-\alpha}=\frac{1}{2} v^{2}\left[s_{2 \alpha}\left(-\lambda_{1} c_{\beta}^{2}+\lambda_{2} s_{\beta}^{2}\right)+\lambda_{345} s_{2 \beta} c_{2 \alpha}-2 \lambda_{5} s_{\beta-\alpha} c_{\beta-\alpha}\right. \\
\left.+2 \lambda_{6} c_{\beta} c_{\beta+2 \alpha}+2 \lambda_{7} s_{\beta} s_{\beta+2 \alpha}\right]
\end{gathered}
$$

where $\lambda_{345} \equiv \lambda_{3}+\lambda_{4}+\lambda_{5}$. We can now eliminate $m_{A}^{2}$ from eqs. (D1) and (D2) and eqs. (10) and (11) using the result of eq. (D3). This yields equations for the other three physical Higgs boson squared-masses and the scalar potential mass parameter $m_{12}^{2}$ in terms of the Higgs scalar quartic couplings

$$
\begin{aligned}
\frac{m_{h}^{2}}{v^{2}} s_{\beta-\alpha}= & -\lambda_{1} c_{\beta}^{3} s_{\alpha}+\lambda_{2} s_{\beta}^{3} c_{\alpha}+\frac{1}{2} \lambda_{345} c_{\beta+\alpha} s_{2 \beta} \\
& +\lambda_{6} c_{\beta}^{2}\left(c_{\beta} c_{\alpha}-3 s_{\beta} s_{\alpha}\right)+\lambda_{7} s_{\beta}^{2}\left(3 c_{\beta} c_{\alpha}-s_{\beta} s_{\alpha}\right) \\
\frac{m_{H}^{2}}{v^{2}} c_{\beta-\alpha}= & \lambda_{1} c_{\beta}^{3} c_{\alpha}+\lambda_{2} s_{\beta}^{3} s_{\alpha}+\frac{1}{2} \lambda_{345} s_{\beta+\alpha} s_{2 \beta} \\
& +\lambda_{6} c_{\beta}^{2}\left(3 s_{\beta} c_{\alpha}+c_{\beta} s_{\alpha}\right)+\lambda_{7} s_{\beta}^{2}\left(s_{\beta} c_{\alpha}+3 c_{\beta} s_{\alpha}\right) \\
\frac{2 m_{H^{ \pm}}^{2}}{v^{2}} s_{\beta-\alpha} c_{\beta-\alpha}= & -s_{2 \alpha}\left(\lambda_{1} c_{\beta}^{2}-\lambda_{2} s_{\beta}^{2}\right)+\lambda_{345} s_{2 \beta} c_{2 \alpha}-\left(\lambda_{4}+\lambda_{5}\right) s_{\beta-\alpha} c_{\beta-\alpha} \\
& +2 \lambda_{6} c_{\beta} c_{\beta+2 \alpha}+2 \lambda_{7} s_{\beta} s_{\beta+2 \alpha}, \\
\frac{2 m_{12}^{2}}{v^{2}} s_{\beta-\alpha} c_{\beta-\alpha}= & -\frac{1}{2} s_{2 \beta} s_{2 \alpha}\left(\lambda_{1} c_{\beta}^{2}-\lambda_{2} s_{\beta}^{2}\right)+\frac{1}{2} \lambda_{345} s_{2 \beta}^{2} c_{2 \alpha} \\
& +\lambda_{6} c_{\beta}^{2}\left[3 c_{\beta} s_{\beta} c_{2 \alpha}-c_{\alpha} s_{\alpha}\left(1+2 s_{\beta}^{2}\right)\right]+\lambda_{7} s_{\beta}^{2}\left[3 s_{\beta} c_{\beta} c_{2 \alpha}+c_{\alpha} s_{\alpha}\left(1+2 c_{\beta}^{2}\right)\right] .
\end{aligned}
$$


Note that eq. (D6) is easily derived by inserting eq. (D3) into eq. (111). A related useful result is easily derived from eqs. (D3) and (D5):

$$
\begin{aligned}
\frac{\left(m_{A}^{2}-m_{H}^{2}\right)}{v^{2}} s_{\beta-\alpha}= & \frac{1}{2} s_{2 \beta}\left(-\lambda_{1} c_{\alpha} c_{\beta}+\lambda_{2} s_{\alpha} s_{\beta}+\lambda_{345} c_{\beta+\alpha}\right)-\lambda_{5} s_{\beta-\alpha} \\
& +\lambda_{6} c_{\beta}\left[c_{\beta} c_{\beta+\alpha}-2 s_{\beta}^{2} c_{\alpha}\right]+\lambda_{7} s_{\beta}\left[s_{\beta} c_{\beta+\alpha}+2 c_{\beta}^{2} s_{\alpha}\right] .
\end{aligned}
$$

It is remarkable that the left hand side of eq. (D8) is proportional only to $s_{\beta-\alpha}$ (i.e., the factor of $c_{\beta-\alpha}$ has canceled). As a result, in the decoupling limit where $c_{\beta-\alpha} \rightarrow 0$, we see that $m_{A}^{2}-m_{H}^{2}=\mathcal{O}\left(v^{2}\right)$.

The expressions given in eqs. (D3)-(D6) are quite complicated. These results simplify considerably when expressed in terms of $\lambda, \hat{\lambda}$ and $\lambda_{A}$ [eqs. (25)-(27)]:

$$
\begin{aligned}
& m_{A}^{2}=v^{2}\left[\lambda_{A}+\hat{\lambda}\left(\frac{s_{\beta-\alpha}}{c_{\beta-\alpha}}-\frac{c_{\beta-\alpha}}{s_{\beta-\alpha}}\right)\right], \\
& m_{h}^{2}=v^{2}\left[\lambda-\frac{\hat{\lambda} c_{\beta-\alpha}}{s_{\beta-\alpha}}\right], \\
& m_{H}^{2}=v^{2}\left[\lambda+\frac{\hat{\lambda} s_{\beta-\alpha}}{c_{\beta-\alpha}}\right] .
\end{aligned}
$$

One can then rewrite eq. (D8) as

$$
m_{H}^{2}-m_{A}^{2}=v^{2}\left[\lambda-\lambda_{A}+\frac{\widehat{\lambda} c_{\beta-\alpha}}{s_{\beta-\alpha}}\right] .
$$

We can invert eqs. (D3)-(D7) and solve for any five of the scalar potential parameters in terms of the physical Higgs masses and the remaining three undetermined variables 12, 48, 49]. It is convenient to solve for $\lambda_{1}, \ldots, \lambda_{5}$ in terms of $\lambda_{6}, \lambda_{7}, m_{12}^{2}$ and the Higgs masses. We obtain:

$$
\begin{aligned}
& \lambda_{1}=\frac{m_{H}^{2} c_{\alpha}^{2}+m_{h}^{2} s_{\alpha}^{2}-m_{12}^{2} t_{\beta}}{v^{2} c_{\beta}^{2}}-\frac{3}{2} \lambda_{6} t_{\beta}+\frac{1}{2} \lambda_{7} t_{\beta}^{3} \\
& \lambda_{2}=\frac{m_{H}^{2} s_{\alpha}^{2}+m_{h}^{2} c_{\alpha}^{2}-m_{12}^{2} t_{\beta}^{-1}}{v^{2} s_{\beta}^{2}}+\frac{1}{2} \lambda_{6} t_{\beta}^{-3}-\frac{3}{2} \lambda_{7} t_{\beta}^{-1} \\
& \lambda_{3}=\frac{\left(m_{H}^{2}-m_{h}^{2}\right) c_{\alpha} s_{\alpha}+2 m_{H^{ \pm}}^{2} s_{\beta} c_{\beta}-m_{12}^{2}}{v^{2} s_{\beta} c_{\beta}}-\frac{1}{2} \lambda_{6} t_{\beta}^{-1}-\frac{1}{2} \lambda_{7} t_{\beta} \\
& \lambda_{4}=\frac{\left(m_{A}^{2}-2 m_{H^{ \pm}}^{2}\right) s_{\beta} c_{\beta}+m_{12}^{2}}{v^{2} s_{\beta} c_{\beta}}-\frac{1}{2} \lambda_{6} t_{\beta}^{-1}-\frac{1}{2} \lambda_{7} t_{\beta} \\
& \lambda_{5}=\frac{m_{12}^{2}-m_{A}^{2} s_{\beta} c_{\beta}}{v^{2} s_{\beta} c_{\beta}}-\frac{1}{2} \lambda_{6} t_{\beta}^{-1}-\frac{1}{2} \lambda_{7} t_{\beta} .
\end{aligned}
$$


In addition, the minimization conditions of eqs. (6) and (17) reduce to:

$$
\begin{aligned}
& m_{11}^{2}=-\frac{1}{2 c_{\beta}}\left(m_{H}^{2} c_{\alpha} c_{\beta-\alpha}-m_{h}^{2} s_{\alpha} s_{\beta-\alpha}\right)+m_{12}^{2} t_{\beta}, \\
& m_{22}^{2}=-\frac{1}{2 s_{\beta}}\left(m_{h}^{2} c_{\alpha} s_{\beta-\alpha}+m_{H}^{2} s_{\alpha} c_{\beta-\alpha}\right)+m_{12}^{2} t_{\beta}^{-1} .
\end{aligned}
$$

Note that $\lambda_{6}$ and $\lambda_{7}$ do not appear when $m_{11}^{2}$ and $m_{22}^{2}$ are expressed entirely in terms of $m_{12}^{2}$ and physical Higgs masses.

In some cases, it proves more convenient to eliminate $m_{12}^{2}$ in favor of $\lambda_{5}$ using eq. (D17). The end result is:

$$
\begin{aligned}
& \lambda_{1}=\frac{m_{H}^{2} c_{\alpha}^{2}+m_{h}^{2} s_{\alpha}^{2}-m_{A}^{2} s_{\beta}^{2}}{v^{2} c_{\beta}^{2}}-\lambda_{5} t_{\beta}^{2}-2 \lambda_{6} t_{\beta}, \\
& \lambda_{2}=\frac{m_{H}^{2} s_{\alpha}^{2}+m_{h}^{2} c_{\alpha}^{2}-m_{A}^{2} c_{\beta}^{2}}{v^{2} s_{\beta}^{2}}-\lambda_{5} t_{\beta}^{-2}-2 \lambda_{7} t_{\beta}^{-1}, \\
& \lambda_{3}=\frac{\left(m_{H}^{2}-m_{h}^{2}\right) s_{\alpha} c_{\alpha}+\left(2 m_{H^{ \pm}}^{2}-m_{A}^{2}\right) s_{\beta} c_{\beta}}{v^{2} s_{\beta} c_{\beta}}-\lambda_{5}-\lambda_{6} t_{\beta}^{-1}-\lambda_{7} t_{\beta}, \\
& \lambda_{4}=\frac{2\left(m_{A}^{2}-m_{H^{ \pm}}^{2}\right)}{v^{2}}+\lambda_{5},
\end{aligned}
$$

and

$$
\begin{aligned}
& m_{11}^{2}=-\frac{1}{2 c_{\beta}}\left(m_{H}^{2} c_{\alpha} c_{\beta-\alpha}-m_{h}^{2} s_{\alpha} s_{\beta-\alpha}\right)+\left(m_{A}^{2}+\lambda_{5} v^{2}\right) s_{\beta}^{2}+\frac{1}{2} v^{2}\left(\lambda_{6} s_{\beta} c_{\beta}+\lambda_{7} s_{\beta}^{2} t_{\beta}\right) \\
& m_{22}^{2}=-\frac{1}{2 s_{\beta}}\left(m_{h}^{2} c_{\alpha} s_{\beta-\alpha}+m_{H}^{2} s_{\alpha} c_{\beta-\alpha}\right)+\left(m_{A}^{2}+\lambda_{5} v^{2}\right) c_{\beta}^{2}+\frac{1}{2} v^{2}\left(\lambda_{6} c_{\beta}^{2} t_{\beta}^{-1}+\lambda_{7} s_{\beta} c_{\beta}\right) .
\end{aligned}
$$

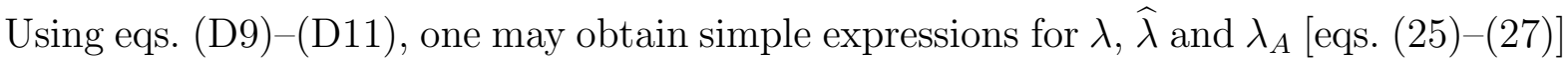
in terms of the neutral Higgs squared-masses:

$$
\begin{aligned}
\lambda v^{2} & =m_{h}^{2} s_{\beta-\alpha}^{2}+m_{H}^{2} c_{\beta-\alpha}^{2}, \\
\hat{\lambda} v^{2} & =\left(m_{H}^{2}-m_{h}^{2}\right) s_{\beta-\alpha} c_{\beta-\alpha}, \\
\lambda_{A} v^{2} & =m_{A}^{2}+\left(m_{H}^{2}-m_{h}^{2}\right)\left(c_{\beta-\alpha}^{2}-s_{\beta-\alpha}^{2}\right), \\
\lambda_{F} v^{2} & =2\left(m_{H^{ \pm}}^{2}-m_{A}^{2}\right),
\end{aligned}
$$

where we have also included an expression for $\lambda_{F} \equiv \lambda_{5}-\lambda_{4}$ in terms of the Higgs squaredmasses [see eq. (11)]. Thus, four of the the invariant coupling parameters can be expressed 
in terms of the physical Higgs masses and the basis-independent quantity $\beta-\alpha$ (see Appendix E).

Finally, we note that eqs. (D27) and (D28) also yield a simple expression for $\beta-\alpha$, which plays such a central role in the decoupling limit. We find two forms that are noteworthy:

$$
\tan [2(\beta-\alpha)]=\frac{-2 \widehat{\lambda} v^{2}}{m_{A}^{2}-\lambda_{A} v^{2}},
$$

and

$$
\sin [2(\beta-\alpha)]=\frac{2 \widehat{\lambda} v^{2}}{m_{H}^{2}-m_{h}^{2}} .
$$

Indeed, if $\hat{\lambda}=0$ then either $c_{\beta-\alpha}=0$ or $s_{\beta-\alpha}=0$ as discussed in Section 5 . For $\hat{\lambda} \neq 0$, the condition $m_{H}>m_{h}$ implies that $\hat{\lambda} s_{\beta-\alpha} c_{\beta-\alpha}>0$. This inequality, when applied to eq. (D9), imposes the following constraint on $m_{A}$

$$
v^{2}\left[\lambda_{A}-\frac{2 \widehat{\lambda} c_{\beta-\alpha}}{s_{\beta-\alpha}}\right]<m_{A}^{2}<v^{2}\left[\lambda_{A}+\frac{2 \widehat{\lambda} s_{\beta-\alpha}}{c_{\beta-\alpha}}\right] .
$$

In addition, we require that $m_{A}^{2} \geq 0$.

The expressions for the Higgs masses [eqs. (D9)-(D11)] and $\beta-\alpha$ [eq. (D30) or (D31)] are especially useful when considering the approach to the decoupling limit, where $\left|c_{\beta-\alpha}\right| \ll 1$. For example, eqs. (D9)-(D11) reduce in this limit to the results of eqs. (29)-(31). Moreover, $\sin [2(\beta-\alpha)] \simeq-\tan [2(\beta-\alpha)] \simeq 2 c_{\beta-\alpha}$, and eqs. (D30) and (D31) reduce to the results given by eq. (36). The corresponding results in limiting case of $\left|s_{\beta-\alpha}\right| \ll 1$ treated in Section 5 are also similarly obtained.

\section{APPENDIX E: INVARIANT COMBINATIONS OF THE HIGGS SCALAR PO- TENTIAL PARAMETERS}

In the most general $2 \mathrm{HDM}$ model, there is no distinction between the two $Y=1$ complex doublets, $\Phi_{1}$ and $\Phi_{2}$. In principle, one could choose any two orthogonal linear combinations of $\Phi_{1}$ and $\Phi_{2}$ (i.e., choose a new basis for the scalar doublets), and construct the scalar sector Lagrangian with respect to the new basis. Clearly, the parameters of eq. (11), $m_{i j}^{2}$ and the $\lambda_{i}$, would all be modified, along with $\alpha$ and $\beta$. However, there exists seven invariant combinations of the $\lambda_{i}$ that are independent of basis choice [50]. These are: $\lambda, \hat{\lambda}, \lambda_{A}, \lambda_{F}$ defined in eqs. (25)-(28), and $\lambda_{T}, \lambda_{U}$ and $\lambda_{V}$ defined in eqs. (52)-(54). In addition, the 
combination $\beta-\alpha$ is clearly basis independent. Thus, all physical Higgs masses and Higgs self-couplings can be expressed in terms of the above invariant coupling parameters and $\beta-\alpha$. In Appendix D, we have already shown how to express the Higgs masses in terms of the invariant parameters. In Appendices $\mathbf{E}$ and $\mathrm{G}$ we also exhibit the three-Higgs and four-Higgs couplings in terms of the invariant parameters. ${ }^{31}$

To obtain expressions for the Higgs self-couplings in terms of invariant parameters, one must invert the relations between the $\lambda_{i}$ and the invariant coupling parameters. The end result is:

$$
\begin{aligned}
\lambda_{1} & =c_{\beta}^{2}\left(1+3 s_{\beta}^{2}\right) \lambda+2 s_{2 \beta}\left(c_{\beta}^{2} \hat{\lambda}+s_{\beta}^{2} \lambda_{U}\right)-\frac{1}{2} s_{2 \beta}^{2}\left(2 \lambda_{A}-\lambda_{T}\right)+s_{\beta}^{4} \lambda_{V}, \\
\lambda_{2} & =s_{\beta}^{2}\left(1+3 c_{\beta}^{2}\right) \lambda-2 s_{2 \beta}\left(s_{\beta}^{2} \widehat{\lambda}+c_{\beta}^{2} \lambda_{U}\right)-\frac{1}{2} s_{2 \beta}^{2}\left(2 \lambda_{A}-\lambda_{T}\right)+c_{\beta}^{4} \lambda_{V}, \\
\lambda_{345} & =\left(2 c_{2 \beta}^{2}-c_{\beta}^{2} s_{\beta}^{2}\right) \lambda-3 s_{2 \beta} c_{2 \beta}\left(\widehat{\lambda}-\lambda_{U}\right)-\left(c_{2 \beta}^{2}-2 c_{\beta}^{2} s_{\beta}^{2}\right)\left(2 \lambda_{A}-\lambda_{T}\right)+\frac{3}{4} s_{2 \beta}^{2} \lambda_{V}, \\
\lambda_{5} & =\left(c_{2 \beta}^{2}+c_{\beta}^{2} s_{\beta}^{2}\right) \lambda-s_{2 \beta} c_{2 \beta}\left(\widehat{\lambda}-\lambda_{U}\right)-c_{2 \beta}^{2} \lambda_{A}+\frac{1}{4} s_{2 \beta}^{2}\left(\lambda_{V}-2 \lambda_{T}\right), \\
\lambda_{6} & =\frac{1}{2} s_{2 \beta}\left(3 s_{\beta}^{2}-1\right) \lambda-c_{\beta} c_{3 \beta} \widehat{\lambda}-s_{\beta} s_{3 \beta} \lambda_{U}+\frac{1}{2} s_{2 \beta} c_{2 \beta}\left(2 \lambda_{A}-\lambda_{T}\right)-\frac{1}{2} s_{\beta}^{2} s_{2 \beta} \lambda_{V}, \\
\lambda_{7} & =\frac{1}{2} s_{2 \beta}\left(3 c_{\beta}^{2}-1\right) \lambda-s_{\beta} s_{3 \beta} \hat{\lambda}-c_{\beta} c_{3 \beta} \lambda_{U}-\frac{1}{2} s_{2 \beta} c_{2 \beta}\left(2 \lambda_{A}-\lambda_{T}\right)-\frac{1}{2} c_{\beta}^{2} s_{2 \beta} \lambda_{V},
\end{aligned}
$$

and $\lambda_{4}=\lambda_{5}-\lambda_{F}$.

The significance of the invariant coupling parameters is most evident in the so-called Higgs basis of ref. [44], in which only the neutral component of one of the two Higgs doublets (say, the first one) possesses a vacuum expectation value. Let us denote the two Higgs doublets in this basis by $\Phi_{a}$ and $\Phi_{b}$. Then, after a rotation from the $\Phi_{1}-\Phi_{2}$ basis by an angle $\beta$,

$$
\begin{aligned}
& \Phi_{a}=\Phi_{1} \cos \beta+\Phi_{2} \sin \beta, \\
& \Phi_{b}=-\Phi_{1} \sin \beta+\Phi_{2} \cos \beta,
\end{aligned}
$$

one obtains

$$
\Phi_{a}=\left(\begin{array}{c}
G^{+} \\
\frac{1}{\sqrt{2}}\left(v+\varphi_{a}^{0}+i G^{0}\right)
\end{array}\right), \quad \Phi_{b}=\left(\begin{array}{c}
H^{+} \\
\frac{1}{\sqrt{2}}\left(\varphi_{b}^{0}+i A\right)
\end{array}\right)
$$

31 The Higgs couplings to vector bosons depend only on $\beta-\alpha$ [see eqs. (37)- 339]. The Higgs couplings to fermions in the Type-III model (in which both up and down-type fermions couple to both Higgs doublets) can also be written in terms of invariant parameters. However, one would then have to identify the appropriate invariant combinations of the Higgs-fermion Yukawa coupling parameters [50], $\eta_{i}^{U}$ and $\eta_{i}^{D}$ [see eq. 421]. 
where $\varphi_{a}^{0}$ and $\varphi_{b}^{0}$ are related in the CP-conserving model to the CP-even neutral Higgs bosons by:

$$
\begin{aligned}
H & =\varphi_{a}^{0} \cos (\beta-\alpha)-\varphi_{b}^{0} \sin (\beta-\alpha), \\
h & =\varphi_{a}^{0} \sin (\beta-\alpha)+\varphi_{b}^{0} \cos (\beta-\alpha) .
\end{aligned}
$$

Here, we see that $\beta-\alpha$ is the invariant angle that characterizes the direction of the CPeven mass eigenstates (in the two-dimensional Higgs "flavor" space) relative to that of the vacuum expectation value.

In the Higgs basis, the corresponding values of $\lambda_{1}, \cdots, \lambda_{7}$ are easily evaluated by putting $\beta=0$ in eq. (E1). Thus, the scalar potential takes the following form:

$$
\begin{aligned}
\mathcal{V}= & m_{a a}^{2} \Phi_{a}^{\dagger} \Phi_{a}+m_{b b}^{2} \Phi_{b}^{\dagger} \Phi_{b}-\left[m_{a b}^{2} \Phi_{a}^{\dagger} \Phi_{b}+\text { h.c. }\right] \\
& +\frac{1}{2} \lambda\left(\Phi_{a}^{\dagger} \Phi_{a}\right)^{2}+\frac{1}{2} \lambda_{V}\left(\Phi_{b}^{\dagger} \Phi_{b}\right)^{2}+\left(\lambda_{T}+\lambda_{F}\right)\left(\Phi_{a}^{\dagger} \Phi_{a}\right)\left(\Phi_{b}^{\dagger} \Phi_{b}\right)+\left(\lambda-\lambda_{A}-\lambda_{F}\right)\left(\Phi_{a}^{\dagger} \Phi_{b}\right)\left(\Phi_{b}^{\dagger} \Phi_{a}\right) \\
& +\left\{\frac{1}{2}\left(\lambda-\lambda_{A}\right)\left(\Phi_{a}^{\dagger} \Phi_{b}\right)^{2}-\left[\widehat{\lambda}\left(\Phi_{a}^{\dagger} \Phi_{a}\right)+\lambda_{U}\left(\Phi_{b}^{\dagger} \Phi_{b}\right)\right] \Phi_{a}^{\dagger} \Phi_{b}+\text { h.c. }\right\}
\end{aligned}
$$

where three new invariant quantities are revealed:

$$
\begin{aligned}
& m_{a a}^{2}=m_{11}^{2} c_{\beta}^{2}+m_{22}^{2} s_{\beta}^{2}-\left[m_{12}^{2}+\left(m_{12}^{*}\right)^{2}\right] s_{\beta} c_{\beta}, \\
& m_{b b}^{2}=m_{11}^{2} s_{\beta}^{2}+m_{22}^{2} c_{\beta}^{2}+\left[m_{12}^{2}+\left(m_{12}^{*}\right)^{2}\right] s_{\beta} c_{\beta}, \\
& m_{a b}^{2}=\left(m_{11}^{2}-m_{22}^{2}\right) s_{\beta} c_{\beta}+m_{12}^{2} c_{\beta}^{2}-\left(m_{12}^{*}\right)^{2} s_{\beta}^{2} .
\end{aligned}
$$

In the CP-conserving theory where $m_{12}^{2}$ is real, the corresponding potential minimum conditions [eqs. (6) -(17)] simplify to:

$$
m_{a a}^{2}=-\frac{1}{2} v^{2} \lambda, \quad m_{a b}^{2}=-\frac{1}{2} v^{2} \widehat{\lambda}
$$

with no constraint on $m_{b b}^{2}$. In fact, $m_{b b}^{2}$ is related to $m_{A}^{2}$ :

$$
\begin{aligned}
m_{A}^{2} & =\operatorname{Tr} m^{2}+\frac{1}{2} v^{2}\left(\lambda+\lambda_{T}\right) \\
& =m_{b b}^{2}+\frac{1}{2} v^{2} \lambda_{T}
\end{aligned}
$$

after imposing the potential minimum condition [eq. (E10)]. It is convenient to trade the free parameter $m_{b b}^{2}$ for $\beta-\alpha$. Using the results of eqs. (D30) and (D31), it follows that

$$
\tan [2(\beta-\alpha)]=\frac{2 \widehat{\lambda}}{\lambda_{A}-\frac{1}{2} \lambda_{T}-m_{b b}^{2} / v^{2}},
$$


where the sign of $\sin [2(\beta-\alpha)]$ is equal to the sign of $\widehat{\lambda}$.

It is now straightforward to obtain the three-Higgs and four-Higgs couplings in terms of the invariant coupling parameters and $\beta-\alpha$, by inserting eqs. (E3)-(E5) into eq. (E6)

\section{APPENDIX F: THREE-HIGGS VERTICES IN THE TWO-HIGGS DOUBLET} MODEL

In this Appendix, we list the Feynman rules for the three-point Higgs interaction in the most general CP-conserving two-Higgs doublet extension of the Standard Model. The Feynman rule for the $A B C$ vertex is denoted by $i g_{A B C} \cdot{ }^{32}$ For completeness, $R$-gauge Feynman rules involving the Goldstone bosons $\left(G^{ \pm}\right.$and $\left.G\right)$ are also listed.

The Feynman rules are obtained from the scalar potential by multiplying the corresponding coefficients of $\mathcal{V}$ by $-i$ times the appropriate symmetry factor. To obtain the three-Higgs couplings in terms of $\beta-\alpha$ and the invariant coupling parameters, we insert eqs. (E3) $-(\mathrm{E} 5)$ into eq. (E6), and identify the terms that are cubic in the Higgs boson fields. The resulting three-point Higgs couplings (which are proportional to $v \equiv 2 m_{W} / g$ ) are given by:

$$
\begin{aligned}
g_{h A A}= & -v\left[\lambda_{T} s_{\beta-\alpha}-\lambda_{U} c_{\beta-\alpha}\right], \\
g_{H A A}= & -v\left[\lambda_{T} c_{\beta-\alpha}+\lambda_{U} s_{\beta-\alpha}\right], \\
g_{h H H}=3 v\left[\lambda s_{\beta-\alpha}\left(-\frac{2}{3}+c_{\beta-\alpha}^{2}\right)+\widehat{\lambda} c_{\beta-\alpha}\left(1-3 s_{\beta-\alpha}^{2}\right)\right. & \left.\quad+\left(2 \lambda_{A}-\lambda_{T}\right) s_{\beta-\alpha}\left(\frac{1}{3}-c_{\beta-\alpha}^{2}\right)+\lambda_{U} s_{\beta-\alpha}^{2} c_{\beta-\alpha}\right], \\
g_{H h h}=3 v\left[\lambda c_{\beta-\alpha}\left(-\frac{2}{3}+s_{\beta-\alpha}^{2}\right)-\hat{\lambda} s_{\beta-\alpha}\left(1-3 c_{\beta-\alpha}^{2}\right)\right. & \left.\quad+\left(2 \lambda_{A}-\lambda_{T}\right) c_{\beta-\alpha}\left(\frac{1}{3}-s_{\beta-\alpha}^{2}\right)-\lambda_{U} c_{\beta-\alpha}^{2} s_{\beta-\alpha}\right], \\
g_{h h h}= & -3 v\left[\lambda s_{\beta-\alpha}\left(1+c_{\beta-\alpha}^{2}\right)-3 \widehat{\lambda} c_{\beta-\alpha} s_{\beta-\alpha}^{2}-\left(2 \lambda_{A}-\lambda_{T}\right) s_{\beta-\alpha} c_{\beta-\alpha}^{2}-\lambda_{U} c_{\beta-\alpha}^{3}\right], \\
g_{H H H}= & -3 v\left[\lambda c_{\beta-\alpha}\left(1+s_{\beta-\alpha}^{2}\right)+3 \widehat{\lambda} s_{\beta-\alpha} c_{\beta-\alpha}^{2}-\left(2 \lambda_{A}-\lambda_{T}\right) c_{\beta-\alpha} s_{\beta-\alpha}^{2}+\lambda_{U} s_{\beta-\alpha}^{3}\right], \\
g_{h H^{+} H^{-}}= & -v\left[\left(\lambda_{T}+\lambda_{F}\right) s_{\beta-\alpha}-\lambda_{U} c_{\beta-\alpha}\right], \\
g_{H H^{+} H^{-}}= & -v\left[\left(\lambda_{T}+\lambda_{F}\right) c_{\beta-\alpha}+\lambda_{U} s_{\beta-\alpha}\right] .
\end{aligned}
$$

32 To obtain $g_{A B C}$, multiply the coefficient of $A B C$ that appears in the interaction Lagrangian by the appropriate symmetry factor $n$ !, where $n$ is the number of identical particles at the vertex. Note that $H^{+}$ and $H^{-}$are not considered identical. 
TABLE I: Three-Higgs vertex Feynman rules in the approach to the decoupling limit are given by $i g_{A B C}=i v\left(X_{A B C}+Y_{A B C} c_{\beta-\alpha}\right)$, where the coefficients $X$ and $Y$ are listed below.

\begin{tabular}{|l|c|c|}
\hline$A B C$ & $X_{A B C}$ & $Y_{A B C}$ \\
\hline$h h h$ & $-3 \lambda$ & $9 \widehat{\lambda}$ \\
$h h H$ & $-3 \widehat{\lambda}$ & $\lambda+2\left(\lambda_{T}-2 \lambda_{A}\right)$ \\
$h H H$ & $2\left(\lambda_{A}-\lambda\right)-\lambda_{T}$ & $3\left(\lambda_{U}-2 \widehat{\lambda}\right)$ \\
$h A A$ & $-\lambda_{T}$ & $\lambda_{U}$ \\
$h H^{+} H^{-}$ & $-\lambda_{T}-\lambda_{F}$ & $\lambda_{U}$ \\
$H H H$ & $-3 \lambda_{U}$ & $6\left(\lambda_{A}-\lambda\right)-3 \lambda_{T}$ \\
$H A A$ & $-\lambda_{U}$ & $-\lambda_{T}$ \\
$H H^{+} H^{-}$ & $-\lambda_{U}$ & $-\lambda_{T}-\lambda_{F}$ \\
\hline
\end{tabular}

In the approach to the decoupling limit, the three-Higgs vertices simplify considerably as exhibited in Table I. Here, we have listed all the cubic couplings in the form:

$$
g_{A B C}=v\left(X_{A B C}+Y_{A B C} c_{\beta-\alpha}\right),
$$

where the coefficients $X$ and $Y$ are given in terms of various linear combinations of the invariant coupling parameters. These results follow trivially from eq. (F1).

The couplings involving the Goldstone bosons are given by

$$
\begin{aligned}
g_{h G G} & =g_{h G^{+} G^{-}}=v\left[\hat{\lambda} c_{\beta-\alpha}-\lambda s_{\beta-\alpha}\right], \\
g_{H G G} & =g_{H G^{+} G^{-}}=-v\left[\widehat{\lambda} s_{\beta-\alpha}+\lambda c_{\beta-\alpha}\right], \\
g_{h A G} & =v\left[\widehat{\lambda} s_{\beta-\alpha}-\left(\lambda-\lambda_{A}\right) c_{\beta-\alpha}\right], \\
g_{H A G} & =v\left[\widehat{\lambda} c_{\beta-\alpha}+\left(\lambda-\lambda_{A}\right) s_{\beta-\alpha}\right], \\
g_{h H^{ \pm} G^{\mp}} & =v\left[\widehat{\lambda} s_{\beta-\alpha}-\left(\lambda-\lambda_{A}-\frac{1}{2} \lambda_{F}\right) c_{\beta-\alpha}\right], \\
g_{H H^{ \pm} G^{\mp}} & =v\left[\widehat{\lambda} c_{\beta-\alpha}+\left(\lambda-\lambda_{A}-\frac{1}{2} \lambda_{F}\right) s_{\beta-\alpha}\right], \\
g_{A H^{ \pm} G^{\mp}} & = \pm \frac{i}{2} v \lambda_{F} .
\end{aligned}
$$


In the rule for the $A H^{ \pm} G^{\mp}$ vertex, the sign corresponds to $H^{ \pm}$entering the vertex and $G^{ \pm}$ leaving the vertex.

One can also express the three-Higgs vertices in terms of the Higgs masses by using eqs. (D26) -(D29). The Feynman rules for the three-point Higgs vertices that involve Goldstone bosons then take on rather simple forms:

$$
\begin{aligned}
g_{h G G} & =g_{h G^{+} G^{-}}=\frac{-g}{2 m_{W}} m_{h}^{2} s_{\beta-\alpha}, \\
g_{H G G} & =g_{H G^{+} G^{-}}=\frac{-g}{2 m_{W}} m_{H}^{2} c_{\beta-\alpha}, \\
g_{h A G} & =\frac{-g}{2 m_{W}}\left(m_{h}^{2}-m_{A}^{2}\right) c_{\beta-\alpha}, \\
g_{H A G} & =\frac{g}{2 m_{W}}\left(m_{H}^{2}-m_{A}^{2}\right) s_{\beta-\alpha}, \\
g_{h H^{ \pm} G^{\mp}} & =\frac{g}{2 m_{W}}\left(m_{H^{ \pm}}^{2}-m_{h}^{2}\right) c_{\beta-\alpha}, \\
g_{H H^{ \pm} G^{\mp}} & =\frac{-g}{2 m_{W}}\left(m_{H^{ \pm}}^{2}-m_{H}^{2}\right) s_{\beta-\alpha}, \\
g_{A H^{ \pm} G^{\mp}} & =\frac{ \pm i g}{2 m_{W}}\left(m_{H^{ \pm}}^{2}-m_{A}^{2}\right) .
\end{aligned}
$$

The cubic couplings of the physical Higgs bosons, expressed in terms of the Higgs masses, are more complicated. For example, let us first compute $g_{h h h}$ in terms of $\lambda_{1}, \cdots, \lambda_{7}$ :

$$
g_{h h h}=3 v\left[\lambda_{1} s_{\alpha}^{3} c_{\beta}-\lambda_{2} c_{\alpha}^{3} s_{\beta}+\lambda_{345} s_{\alpha} c_{\alpha} c_{\alpha+\beta}-\lambda_{6} s_{\alpha}^{2}\left(3 c_{\alpha} c_{\beta}-s_{\alpha} s_{\beta}\right)+\lambda_{7} c_{\alpha}^{2}\left(3 s_{\alpha} s_{\beta}-c_{\alpha} c_{\beta}\right)\right] .
$$

This can then be re-expressed in terms of the Higgs masses using eqs. (D20)-(D23). The end result is 12 ]

$$
g_{h h h}=-3 v\left[\frac{m_{h}^{2} s_{\beta-\alpha}}{v^{2}}+\left(\frac{m_{h}^{2}-m_{A}^{2}-\lambda_{5} v^{2}}{v^{2} s_{\beta} c_{\beta}}\right) c_{\beta-\alpha}^{2} c_{\beta+\alpha}+\left(\lambda_{6} \frac{s_{\alpha}}{s_{\beta}}-\lambda_{7} \frac{c_{\alpha}}{c_{\beta}}\right) c_{\beta-\alpha}^{2}\right] .
$$

Note that the decoupling limit result [eq. (57)] follows easily after using eq. (29) to obtain the $\mathcal{O}\left(c_{\beta-\alpha}\right)$ correction. We have also exhibited $g_{h H^{+} H^{-}}$in eq. (59). Expressions for the other three-Higgs couplings in terms of the Higgs masses can be found in ref. [12] (see also ref. [48] for the case of $\lambda_{6}=\lambda_{7}=0$ and ref. [51] for other special cases). However, in the most general case, such expressions are less useful. Finally, using eq. (D29) we note the relations

$$
\begin{aligned}
v\left[g_{h H^{+} H^{-}}-g_{h A A}\right] & =-2\left(m_{H^{ \pm}}^{2}-m_{A}^{2}\right) s_{\beta-\alpha}, \\
v\left[g_{H H^{+} H^{-}}-g_{H A A}\right] & =-2\left(m_{H^{ \pm}}^{2}-m_{A}^{2}\right) c_{\beta-\alpha} .
\end{aligned}
$$




\section{APPENDIX G: FOUR-HIGGS VERTICES IN THE TWO-HIGGS DOUBLET MODEL}

In this Appendix, we list the Feynman rules for the four-point Higgs interaction in the most general CP-conserving two-Higgs doublet extension of the Standard Model. Recalling that $\mathcal{L}_{\text {int }} \ni-\mathcal{V}$, the Feynman rules are obtained from the scalar potential ${ }^{33}$ by multiplying the corresponding coefficients of $\mathcal{V}$ by $-i$ times the appropriate symmetry factor. We find it convenient to write the terms of the potential that are quartic in the Higgs fields as a sum of two pieces: $\mathcal{V} \ni \mathcal{V}_{A}+\mathcal{V}_{B}$, where $\mathcal{V}_{A}$ depends explicitly on $\beta-\alpha$ and $\mathcal{V}_{B}$ is independent of $\beta-\alpha$. To obtain the four-Higgs couplings in terms of $\beta-\alpha$ and the invariant coupling parameters, we insert eqs. (E3) $-(\mathrm{E} 5)$ into eq. (E6), and identify the terms that are quartic in the Higgs boson fields. For completeness, the quartic interaction terms involving the Goldstone bosons $\left(G^{ \pm}\right.$and $G$ ) are also listed. The end result is:

$$
\begin{aligned}
& 8 \mathcal{V}_{A}= \\
& h^{4}\left[\lambda s_{\beta-\alpha}^{2}\left(3 c_{\beta-\alpha}^{2}+1\right)-4 \widehat{\lambda} c_{\beta-\alpha} s_{\beta-\alpha}^{3}-2\left(2 \lambda_{A}-\lambda_{T}\right) c_{\beta-\alpha}^{2} s_{\beta-\alpha}^{2}-4 \lambda_{U} c_{\beta-\alpha}^{3} s_{\beta-\alpha}+\lambda_{V} c_{\beta-\alpha}^{4}\right] \\
& +4 h^{3} H\left[\lambda s_{\beta-\alpha} c_{\beta-\alpha}\left(3 c_{\beta-\alpha}^{2}-1\right)-\widehat{\lambda} s_{\beta-\alpha}^{2}\left(4 c_{\beta-\alpha}^{2}-1\right)-\left(2 \lambda_{A}-\lambda_{T}\right) s_{\beta-\alpha} c_{\beta-\alpha}\left(c_{\beta-\alpha}^{2}-s_{\beta-\alpha}^{2}\right)\right. \\
& \left.+\lambda_{U} c_{\beta-\alpha}^{2}\left(4 s_{\beta-\alpha}^{2}-1\right)-\lambda_{V} s_{\beta-\alpha} c_{\beta-\alpha}^{3}\right] \\
& +2 h^{2} H^{2}\left[\lambda\left(2-9 s_{\beta-\alpha}^{2} c_{\beta-\alpha}^{2}\right)-6\left(\widehat{\lambda}-\lambda_{U}\right) s_{\beta-\alpha} c_{\beta-\alpha}\left(c_{\beta-\alpha}^{2}-s_{\beta-\alpha}^{2}\right)\right. \\
& \left.-\left(2 \lambda_{A}-\lambda_{T}\right)\left(1-6 s_{\beta-\alpha}^{2} c_{\beta-\alpha}^{2}\right)+3 \lambda_{V} s_{\beta-\alpha}^{2} c_{\beta-\alpha}^{2}\right] \\
& +4 h H^{3}\left[\lambda s_{\beta-\alpha} c_{\beta-\alpha}\left(3 s_{\beta-\alpha}^{2}-1\right)+\hat{\lambda} c_{\beta-\alpha}^{2}\left(4 s_{\beta-\alpha}^{2}-1\right)+\left(2 \lambda_{A}-\lambda_{T}\right) s_{\beta-\alpha} c_{\beta-\alpha}\left(c_{\beta-\alpha}^{2}-s_{\beta-\alpha}^{2}\right)\right. \\
& \left.-\lambda_{U} s_{\beta-\alpha}^{2}\left(4 c_{\beta-\alpha}^{2}-1\right)-\lambda_{V} c_{\beta-\alpha} s_{\beta-\alpha}^{3}\right] \\
& +H^{4}\left[\lambda c_{\beta-\alpha}^{2}\left(3 s_{\beta-\alpha}^{2}+1\right)+4 \widehat{\lambda} c_{\beta-\alpha}^{3} s_{\beta-\alpha}-2\left(2 \lambda_{A}-\lambda_{T}\right) c_{\beta-\alpha}^{2} s_{\beta-\alpha}^{2}+4 \lambda_{U} c_{\beta-\alpha} s_{\beta-\alpha}^{3}+\lambda_{V} s_{\beta-\alpha}^{4}\right] \\
& +2 h^{2} A^{2}\left[\lambda_{T} s_{\beta-\alpha}^{2}-2 \lambda_{U} s_{\beta-\alpha} c_{\beta-\alpha}+\lambda_{V} c_{\beta-\alpha}^{2}\right] \\
& +4 h^{2} A G\left[2\left(\lambda-\lambda_{A}\right) s_{\beta-\alpha} c_{\beta-\alpha}-\widehat{\lambda} s_{\beta-\alpha}^{2}-\lambda_{U} c_{\beta-\alpha}^{2}\right] \\
& +2 h^{2} G^{2}\left[\lambda s_{\beta-\alpha}^{2}-2 \widehat{\lambda} s_{\beta-\alpha} c_{\beta-\alpha}+\lambda_{T} c_{\beta-\alpha}^{2}\right] \\
& +4 h^{2} H^{+} H^{-}\left[\left(\lambda_{T}+\lambda_{F}\right) s_{\beta-\alpha}^{2}-2 \lambda_{U} s_{\beta-\alpha} c_{\beta-\alpha}+\lambda_{V} c_{\beta-\alpha}^{2}\right]
\end{aligned}
$$

${ }^{33}$ Note, e.g., that the term proportional to $h A H^{+} G^{-}$in $\mathcal{V}$ corresponds to $H^{+}$and $G^{-}$directed into the vertex, etc. 


$$
\begin{aligned}
& +4\left(h^{2} H^{+} G^{-}+h^{2} H^{-} G^{+}\right)\left[\left(2 \lambda-2 \lambda_{A}-\lambda_{F}\right) s_{\beta-\alpha} c_{\beta-\alpha}-\widehat{\lambda} s_{\beta-\alpha}^{2}-\lambda_{U} c_{\beta-\alpha}^{2}\right] \\
& +4 h^{2} G^{+} G^{-}\left[\lambda s_{\beta-\alpha}^{2}-2 \widehat{\lambda} s_{\beta-\alpha} c_{\beta-\alpha}+\left(\lambda_{T}+\lambda_{F}\right) c_{\beta-\alpha}^{2}\right] \\
& +4 h H A^{2}\left[\left(\lambda_{T}-\lambda_{V}\right) s_{\beta-\alpha} c_{\beta-\alpha}-\lambda_{U}\left(c_{\beta-\alpha}^{2}-s_{\beta-\alpha}^{2}\right)\right] \\
& \left.+8 h H A G\left[\left(\lambda-\lambda_{A}\right)\left(c_{\beta-\alpha}^{2}-s_{\beta-\alpha}^{2}\right)-\left(\widehat{\lambda}-\lambda_{U}\right) s_{\beta-\alpha} c_{\beta-\alpha}\right)\right] \\
& +4 h H G^{2}\left[\left(\lambda-\lambda_{T}\right) s_{\beta-\alpha} c_{\beta-\alpha}-\hat{\lambda}\left(c_{\beta-\alpha}^{2}-s_{\beta-\alpha}^{2}\right)\right] \\
& +8 h H H^{+} H^{-}\left[\left(\lambda_{T}-\lambda_{V}+\lambda_{F}\right) s_{\beta-\alpha} c_{\beta-\alpha}-\lambda_{U}\left(c_{\beta-\alpha}^{2}-s_{\beta-\alpha}^{2}\right)\right] \\
& +4\left(h H H^{+} G^{-}+h H H^{-} G^{+}\right)\left[\left(2 \lambda-2 \lambda_{A}-\lambda_{F}\right)\left(c_{\beta-\alpha}^{2}-s_{\beta-\alpha}^{2}\right)-2\left(\widehat{\lambda}-\lambda_{U}\right) s_{\beta-\alpha} c_{\beta-\alpha}\right] \\
& +8 h H G^{+} G^{-}\left[\left(\lambda-\lambda_{T}-\lambda_{F}\right) s_{\beta-\alpha} c_{\beta-\alpha}-\hat{\lambda}\left(c_{\beta-\alpha}^{2}-s_{\beta-\alpha}^{2}\right)\right] \\
& +2 H^{2} A^{2}\left[\lambda_{T} c_{\beta-\alpha}^{2}+2 \lambda_{U} s_{\beta-\alpha} c_{\beta-\alpha}+\lambda_{V} s_{\beta-\alpha}^{2}\right] \\
& +4 H^{2} A G\left[2\left(\lambda_{A}-\lambda\right) s_{\beta-\alpha} c_{\beta-\alpha}-\hat{\lambda} c_{\beta-\alpha}^{2}-\lambda_{U} s_{\beta-\alpha}^{2}\right] \\
& +2 H^{2} G^{2}\left[\lambda c_{\beta-\alpha}^{2}+2 \widehat{\lambda} s_{\beta-\alpha} c_{\beta-\alpha}+\lambda_{T} s_{\beta-\alpha}^{2}\right] \\
& +4 H^{2} H^{+} H^{-}\left[\left(\lambda_{T}+\lambda_{F}\right) c_{\beta-\alpha}^{2}+2 \lambda_{U} s_{\beta-\alpha} c_{\beta-\alpha}+\lambda_{V} s_{\beta-\alpha}^{2}\right] \\
& +4\left(H^{2} H^{+} G^{-}+H^{2} H^{-} G^{+}\right)\left[\left(2 \lambda_{A}-2 \lambda+\lambda_{F}\right) s_{\beta-\alpha} c_{\beta-\alpha}-\hat{\lambda} c_{\beta-\alpha}^{2}-\lambda_{U} s_{\beta-\alpha}^{2}\right] \\
& +4 H^{2} G^{+} G^{-}\left[\lambda c_{\beta-\alpha}^{2}+2 \widehat{\lambda} s_{\beta-\alpha} c_{\beta-\alpha}+\left(\lambda_{T}+\lambda_{F}\right) s_{\beta-\alpha}^{2}\right] \\
& +4 i\left[h A H^{+} G^{-}-h A H^{-} G^{+}+H G H^{+} G^{-}-H G H^{-} G^{+}\right] \lambda_{F} s_{\beta-\alpha} \\
& -4 i\left[h G H^{+} G^{-}-h G H^{-} G^{+}-H A H^{+} G^{-}+H A H^{-} G^{+}\right] \lambda_{F} c_{\beta-\alpha},
\end{aligned}
$$

and

$$
\begin{aligned}
8 \mathcal{V}_{B} & =\lambda_{V}\left(A^{4}+4 A^{2} H^{+} H^{-}+4 H^{+} H^{-} H^{+} H^{-}\right) \\
& -4 \lambda_{U}\left(A^{3} G+A^{2} H^{+} G^{-}+A^{2} H^{-} G^{+}+2 A G H^{+} H^{-}+2 H^{+} H^{-} H^{+} G^{-}+2 H^{+} H^{-} H^{-} G^{+}\right) \\
& +2\left[2\left(\lambda-\lambda_{A}\right)+\lambda_{T}\right] A^{2} G^{2} \\
& +4\left(\lambda_{T}+\lambda_{F}\right)\left(A^{2} G^{+} G^{-}+G^{2} H^{+} H^{-}\right) \\
& -4 \widehat{\lambda}\left(A G^{3}+2 A G G^{+} G^{-}+G^{2} H^{+} G^{-}+G^{2} H^{-} G^{+}+2 H^{+} G^{+} G^{-} G^{-}+2 H^{-} G^{-} G^{+} G^{+}\right) \\
& +4\left[2\left(\lambda-\lambda_{A}\right)-\lambda_{F}\right]\left(A G H^{+} G^{-}+A G H^{-} G^{+}\right) \\
& +\lambda\left(G^{4}+4 G^{2} G^{+} G^{-}+4 G^{+} G^{-} G^{+} G^{-}\right) \\
& +4\left(\lambda-\lambda_{A}\right)\left(H^{+} H^{+} G^{-} G^{-}+H^{-} H^{-} G^{+} G^{+}\right) \\
& +8\left(\lambda-\lambda_{A}+\lambda_{T}\right) H^{+} H^{-} G^{+} G^{-} .
\end{aligned}
$$


The quartic Higgs couplings are now easily obtained by including the appropriate symmetry factors. For example, the $h^{4}$ and $H^{4}$ couplings are given by

$$
\begin{gathered}
g_{h h h h}=-3\left[\lambda s_{\beta-\alpha}^{2}\left(1+3 c_{\beta-\alpha}^{2}\right)-4 \widehat{\lambda} c_{\beta-\alpha} s_{\beta-\alpha}^{3}-2\left(2 \lambda_{A}-\lambda_{T}\right) c_{\beta-\alpha}^{2} s_{\beta-\alpha}^{2}\right. \\
\left.-4 \lambda_{U} c_{\beta-\alpha}^{3} s_{\beta-\alpha}+\lambda_{V} c_{\beta-\alpha}^{4}\right], \\
g_{H H H H}=-3\left[\lambda c_{\beta-\alpha}^{2}\left(1+3 s_{\beta-\alpha}^{2}\right)+4 \widehat{\lambda}_{\beta-\alpha}^{3} s_{\beta-\alpha}-2\left(2 \lambda_{A}-\lambda_{T}\right) c_{\beta-\alpha}^{2} s_{\beta-\alpha}^{2}\right. \\
\left.+4 \lambda_{U} c_{\beta-\alpha} s_{\beta-\alpha}^{3}+\lambda_{V} s_{\beta-\alpha}^{4}\right] .
\end{gathered}
$$

Note the first appearance of physical observables that depend on $\lambda_{V}$.

Let us denote the Feynman rule for the $A B C D$ vertex by $i g_{A B C D}$. In the approach to the decoupling limit, the four-Higgs vertices simplify considerably as exhibited in Table II. Here, we have listed all couplings in the form:

$$
g_{A B C D}=\left(X_{A B C D}+Y_{A B C D} c_{\beta-\alpha}\right),
$$

where the coefficients $X$ and $Y$ are given in terms of various linear combinations of the invariant coupling parameters. Note that the terms contained in $\mathcal{V}_{B}$ are not affected by the decoupling limit since these terms are independent of $\beta-\alpha$.

The four-Higgs couplings can be rewritten in terms of $\lambda_{1}, \cdots, \lambda_{7}, \alpha$ and $\beta$. The resulting expressions are generally more complex, with a few notable exceptions. For example, the quartic couplings in $\mathcal{V}_{A}$ that depend only on $h$ and $H$ are independent of $\beta$

$$
\begin{aligned}
\mathcal{V}_{A} \ni & \frac{1}{8} h^{4}\left[\lambda_{1} s_{\alpha}^{4}+\lambda_{2} c_{\alpha}^{4}+\frac{1}{2} \lambda_{345} s_{2 \alpha}^{2}-2 s_{2 \alpha}\left(\lambda_{6} s_{\alpha}^{2}+\lambda_{7} c_{\alpha}^{2}\right)\right] \\
& +\frac{1}{2} h^{3} H\left[\frac{1}{2} s_{2 \alpha}\left(-\lambda_{1} s_{\alpha}^{2}+\lambda_{2} c_{\alpha}^{2}-\lambda_{345} c_{2 \alpha}\right)+\lambda_{6} s_{\alpha} s_{3 \alpha}+\lambda_{7} c_{\alpha} c_{3 \alpha}\right] \\
& +\frac{1}{4} h^{2} H^{2}\left[\frac{3}{4} s_{2 \alpha}^{2}\left(\lambda_{1}+\lambda_{2}-2 \lambda_{345}\right)+\lambda_{345}-3 s_{2 \alpha} c_{2 \alpha}\left(\lambda_{6}-\lambda_{7}\right)\right] \\
& +\frac{1}{2} h H^{3}\left[\frac{1}{2} s_{2 \alpha}\left(-\lambda_{1} c_{\alpha}^{2}+\lambda_{2} s_{\alpha}^{2}+\lambda_{345} c_{2 \alpha}\right)+\lambda_{6} c_{\alpha} c_{3 \alpha}+\lambda_{7} s_{\alpha} s_{3 \alpha}\right] \\
& +\frac{1}{8} H^{4}\left[\lambda_{1} c_{\alpha}^{4}+\lambda_{2} s_{\alpha}^{4}+\frac{1}{2} \lambda_{345} s_{2 \alpha}^{2}+2 s_{2 \alpha}\left(\lambda_{6} c_{\alpha}^{2}+\lambda_{7} s_{\alpha}^{2}\right)\right]
\end{aligned}
$$

and in this form these results are somewhat simpler than the corresponding expressions in terms of the invariant coupling parameters given in eq. (G1). One can check that the latter can be obtained from eq. (G6) by rotating to the Higgs basis (see discussion in Appendix E). That is, in eq. (G6), let $\alpha \rightarrow \alpha-\beta, \lambda_{1} \rightarrow \lambda, \lambda_{2} \rightarrow \lambda_{V}, \lambda_{345} \rightarrow 2\left(\lambda-\lambda_{A}\right)+\lambda_{T}, \lambda_{6} \rightarrow-\hat{\lambda}$ and $\lambda_{7} \rightarrow-\lambda_{U}$ [cf. eq. (E6) $]$. 
TABLE II: Four-Higgs vertex Feynman rules in the approach to the decoupling limit are given by $i g_{A B C D}=i\left(X_{A B C D}+Y_{A B C D} c_{\beta-\alpha}\right)$, where the coefficients $X$ and $Y$ are listed below. The rules for $A A A A, A A H^{+} H^{-}$, and $H^{+} H^{-} H^{+} H^{-}$are exact (since they are independent of $\beta-\alpha$ ).

\begin{tabular}{|l|c|c|}
\hline$A B C D$ & $X_{A B C D}$ & $Y_{A B C D}$ \\
\hline$h h h h$ & $-3 \lambda$ & $12 \hat{\lambda}$ \\
$h h h H$ & $-3 \hat{\lambda}$ & $3\left(\lambda+\lambda_{T}-2 \lambda_{A}\right)$ \\
$h h H A$ & $2\left(\lambda_{A}-\lambda\right)-\lambda_{T}$ & $6\left(\lambda_{U}-\hat{\lambda}\right)$ \\
$h h H^{+} H^{-}$ & $-\lambda_{T}-\lambda_{F}$ & $2 \lambda_{U}$ \\
$h H H H$ & $-3 \lambda_{U}$ & $2 \lambda_{U}$ \\
$h H A A$ & $-\lambda_{U}$ & $-\lambda_{T}+\lambda_{V}$ \\
$h H H^{+} H^{-}$ & $-\lambda_{U}$ & $\lambda_{V}-\lambda_{T}-\lambda_{F}$ \\
$H H H H$ & $-3 \lambda_{V}$ & $-12 \lambda_{U}$ \\
$H H A A$ & $-\lambda_{V}$ & $-2 \lambda_{U}$ \\
$H H H^{+} H^{-}$ & $-\lambda_{V}$ & $-2 \lambda_{U}$ \\
$A A A A$ & $-3 \lambda_{V}$ & 0 \\
$A A H^{+} H^{-}$ & $-\lambda_{V}$ & 0 \\
$H^{+} H^{-} H^{+} H^{-}$ & $-2 \lambda_{V}$ & 0 \\
\hline
\end{tabular}

One can also express the four-Higgs vertices in terms of the Higgs masses by using eqs. (D20)-(D23). For example 12],

$$
\begin{aligned}
g_{h h h h}=-3[ & \frac{m_{h}^{2}}{v^{2}}\left(s_{\beta-\alpha}-\frac{c_{\beta+\alpha} c_{\beta-\alpha}^{2}}{s_{\beta} c_{\beta}}\right)^{2}+\frac{m_{H}^{2}}{v^{2}}\left(\frac{s_{\alpha} c_{\alpha} c_{\beta-\alpha}}{s_{\beta} c_{\beta}}\right)^{2} \\
& \left.-\frac{m_{A}^{2}+\lambda_{5} v^{2}}{v^{2}}\left(\frac{c_{\beta+\alpha} c_{\beta-\alpha}}{s_{\beta} c_{\beta}}\right)^{2}-\frac{2\left(\lambda_{6} s_{\alpha}^{2}+\lambda_{7} c_{\alpha}^{2}\right) c_{\beta-\alpha}^{2}}{s_{\beta} c_{\beta}}\right] .
\end{aligned}
$$

Note that the decoupling limit result [eq. (58)] follows trivially, after using eqs. (29) and (31) to obtain the $\mathcal{O}\left(c_{\beta-\alpha}\right)$ correction. Expressions for other four-Higgs couplings in terms 
of the Higgs masses can be found in ref. [12] (see also ref. [48] for the case of $\lambda_{6}=\lambda_{7}=0$ and ref. [51] for other special cases). However, in the most general case, such expressions are less useful.

[1] See, e.g., L. Susskind, Phys. Rept. 104 (1984) 181.

[2] E. Witten, Nucl. Phys. B188, 513 (1981); R.K. Kaul, Phys. Lett. 109B, 19 (1982); Pramana 19, $183(1982)$.

[3] H.P. Nilles, Phys. Rept. 110, 1 (1984). H.E. Haber and G.L. Kane, Phys. Rept. 117, 75 (1985); S.P. Martin, hep-ph/9709356.

[4] S. Dimopoulos and H. Georgi, Nucl. Phys. B193, 150 (1981); K. Harada and N. Sakai, Prog. Theor. Phys. 67, 1877 (1982); K. Inoue, A. Kakuto, H. Komatsu and S. Takeshita, Prog. Theor. Phys. 67, 1889 (1982); L. Girardello and M.T. Grisaru, Nucl. Phys. B194, 65 (1982).

[5] E. Farhi and L. Susskind, Phys. Rept. 74, 277 (1981); R.K. Kaul, Rev. Mod. Phys. 55, 449 (1983); C.T. Hill and E.H. Simmons, FERMI-PUB-02/045-T hep-ph/0203079.

[6] I. Antoniadis, Phys. Lett. B246, 377 (1990); N. Arkani-Hamed, S. Dimopoulos and G.R. Dvali, Phys. Lett. B429, 263 (1998); L. Randall and R. Sundrum, Phys. Rev. Lett. 83, 3370 (1999). For a review, see V.A. Rubakov, Phys. Usp. 44, 871 (2001) [Usp. Fiz. Nauk 171, 913 (2001)].

[7] J.F. Gunion, H.E. Haber, G. Kane and S. Dawson, The Higgs Hunter's Guide (Perseus Publishing, Cambridge, MA, 1990).

[8] K. Inoue, A. Kakuto, H. Komatsu, and S. Takeshita, Prog. Theor. Phys. 68, 927 (1982) [E: 70, 330 (1983)]; R. Flores and M. Sher, Annals Phys. 148, 95 (1983).

[9] J.F. Gunion and H.E. Haber, Nucl. Phys. B 272, 1 (1986) [E: B402, 567 (1993)]; B278, 449 (1986).

[10] H.E. Haber and Y. Nir, Nucl. Phys. B335, 363 (1990).

[11] H.E. Haber, in Physics From the Planck Scale to the Electroweak Scale, Proceedings of the US-Polish Workshop, Warsaw, Poland, September 21-24, 1994, edited by P. Nath, T. Taylor, and S. Pokorski (World Scientific, Singapore, 1995) pp. 49-63.

[12] F. Boudjema and A. Semenov, Phys. Rev. D66, 095007 (2002).

[13] M.N. Dubinin and A.V. Semenov, hep-ph/0206205, Eur. J. Phys. C in press.

[14] J.F. Gunion, H.E. Haber and J. Kalinowski, in preparation. 
[15] L.J. Hall and M.B. Wise, Nucl. Phys. B187, 397 (1981).

[16] S.L. Glashow and S. Weinberg, Phys. Rev. D15, 1958 (1977); E.A. Paschos, Phys. Rev. D15, 1966 (1977).

[17] W.S. Hou, Phys. Lett. B296, 179 (1992); D. Chang, W.S. Hou and W.Y. Keung, Phys. Rev. D48, 217 (1993); D. Atwood, L. Reina and A. Soni, Phys. Rev. D55, 3156 (1997).

[18] N.G. Deshpande and E. Ma, Phys. Rev. D18, 2574 (1978); K.S. Babu and E. Ma, Phys. Rev. D31, 2861 (1985) [E: D33, 3471 (1986)].

[19] J.M. Cornwall, D.N. Levin and G. Tiktopoulos, Phys. Rev. Lett. 30 (1973) 1268 [E: 31 (1973) 572]; Phys. Rev. D10, 1145 (1974) [E: D11, 972 (1975)]; C.H. Llewellyn Smith, Phys. Lett. 46B (1973) 233.

[20] B.W. Lee, C. Quigg and H.B. Thacker, Phys. Rev. D16, 1519 (1977).

[21] H.A. Weldon, Phys. Rev. D30, 1547 (1984).

[22] A.G. Akeroyd, A. Arhrib and E.M. Naimi, Phys. Lett. B490 (2000) 119; A. Arhrib, hep-ph/0012353.

[23] S. Kanemura, T. Kubota and E. Takasugi, Phys. Lett. B313, 155 (1993).

[24] P. Ciafaloni and D. Espriu, Phys. Rev. D56, 1752 (1997)

[25] R.A. Diaz, R. Martinez and J.-Alexis Rodriguez, Phys. Rev. D63, 095007 (2001).

[26] A. Sirlin and R. Zucchini, Nucl. Phys. B266, 389 (1986).

[27] M. Luscher and P. Weisz, Phys. Lett. B212, 472 (1988).

[28] T. Appelquist and J. Carazzone, Phys. Rev. D11 (1975) 2856.

[29] I.F. Ginzburg, M. Krawczyk and P. Osland, Nucl. Instrum. Meth. A472, 149 (2001); and in Physics and experiments with future linear $e^{+} e^{-}$colliders, Proceedings of the 5th International Linear Collider Workshop, Batavia, IL, USA, 2000, edited by A. Para and H.E. Fisk (American Institute of Physics, New York, 2001), pp. 304-311 hep-ph/0101331; contribution to the 2nd ECFA/DESY Study, hep-ph/0101208.

[30] S. Kanemura, S. Kiyoura, Y. Okada, E. Senaha and C.P. Yuan, KEK-TH-856 and MSUHEP21119 hep-ph/0211308.

[31] P. Chankowski, T. Farris, B. Grzadkowski, J.F. Gunion, J. Kalinowski and M. Krawczyk, Phys. Lett. B496, 195 (2000).

[32] A. Djouadi, V. Driesen, W. Hollik and J.I. Illana, Eur. Phys. J. C1, 149 (1998); A. Djouadi, Phys. Lett. B435, 101 (1998); C. S. Huang and X.H. Wu, Phys. Rev. D66, 075002 (2002). 
[33] A. Dobado, M.J. Herrero and S. Penaranda, Eur. Phys. J. C7, 313 (1999).

[34] S. Heinemeyer, W. Hollik and G. Weiglein, Eur. Phys. J. C16, 139 (2000).

[35] L.J. Hall, R. Rattazzi and U. Sarid, Phys. Rev. D50, 7048 (1994); R. Hempfling, Phys. Rev. D49, 6168 (1994).

[36] M. Carena, M. Olechowski, S. Pokorski and C.E.M. Wagner, Nucl. Phys. B426, 269 (1994).

[37] A. Bartl, H. Eberl, K. Hikasa, T. Kon, W. Majerotto and Y. Yamada, Phys. Lett. B378, 167 (1996); R.A. Jiménez and J. Solà, Phys. Lett. B389, 53 (1996); J.A. Coarasa, R.A. Jiménez and J. Solà, Phys. Lett. B389, 312 (1996).

[38] D.M. Pierce, J.A. Bagger, K. Matchev, and R. Zhang, Nucl. Phys. B491, 3 (1997).

[39] M. Carena, H.E. Haber, H.E. Logan and S. Mrenna, Phys. Rev. D65, 055005 (2002) [E: D65, $099902(2002)]$.

[40] M. Carena and H.E. Haber, FERMILAB-Pub-02/114-T and SCIPP 02/07 hep-ph/0208209, Prog. Part. Nucl. Phys., in press.

[41] H.E. Haber, M.J. Herrero, H.E. Logan, S. Peñaranda, S. Rigolin and D. Temes, Phys. Rev. D63, 055004 (2001).

[42] C.P. Burgess, J. Matias and M. Pospelov, Int. J. Mod. Phys. A17, 1841 (2002).

[43] L. Lavoura and J.P. Silva, Phys. Rev. D50, 4619 (1994); F.J. Botella and J.P. Silva, Phys. Rev. D51, 3870 (1995).

[44] G.C. Branco, L. Lavoura and J.P. Silva, CP Violation (Oxford University Press, Oxford, England, 1999), chapters 22 and 23.

[45] T.D. Lee, Phys. Rev. D8, 1226 (1973).

[46] J. Velhinho, R. Santos and A. Barroso, Phys. Lett. B322 (1994) 213.

[47] C. Lin, C. Lee and Y.-W. Yang, Phys. Rev. D50, 558 (1994).

[48] M.N. Dubinin and A.V. Semenov, hep-ph/9812246. This paper contains a number of sign errors. For example, in the $h h h h$ coupling, the relative sign between two terms in the coefficient multiplying $m_{h}^{2}$ is incorrect. The correct factor is $c_{\alpha}^{3} c_{\beta}-s_{\alpha}^{3} s_{\beta}=s_{\beta} c_{\beta} s_{\beta-\alpha}+c_{\beta+\alpha} c_{\beta-\alpha}^{2}$. In deriving this last result, one makes use of the remarkable identity: $s_{\beta} c_{\beta}-s_{\alpha} c_{\alpha}=c_{\beta+\alpha} s_{\beta-\alpha}$.

[49] R. Santos, S.M. Oliveira and A. Barroso, hep-ph/0112202.

[50] J.F. Gunion and H.E. Haber, in preparation.

[51] R. Santos and A. Barroso, Phys. Rev. D56, 5366 (1997); L. Brucher and R. Santos, Eur. Phys. J. C12, 87 (2000). 